Florida International University FIU Digital Commons

6-11-2008

\title{
Lattice Boltzmann Modeling of Fluid Flow and Solute Transport in Karst Aquifers
}

Shadab Anwar

Florida International University, sanwa001@fiu.edu

DOI: $10.25148 /$ etd.FI08121901

Follow this and additional works at: https://digitalcommons.fiu.edu/etd

\section{Recommended Citation}

Anwar, Shadab, "Lattice Boltzmann Modeling of Fluid Flow and Solute Transport in Karst Aquifers" (2008). FIU Electronic Theses and Dissertations. 22.

https://digitalcommons.fiu.edu/etd/22 


\section{FLORIDA INTERNATIONAL UNIVERSITY}

Miami, Florida

\section{LATTICE BOLTZMANN MODELING OF FLUID FLOW AND SOLUTE TRANSPORT IN KARST AQUIFERS}

A dissertation submitted in partial fulfillment of the requirements for the degree of DOCTOR OF PHILOSOPHY

in

GEOSCIENCES

by

Shadab Anwar

2008 
To: Dean Kenneth Furton

College of Arts and Sciences

This dissertation, written by Shadab Anwar, and entitled Lattice Boltzmann Modeling of Fluid Flow and Solute Transport in Karst Aquifers, having been approved in respect to style and intellectual content, is referred to you for judgment.

We have read this dissertation and recommend that it be approved.

Dean Whitman

René M. Price

Yong X. Tao

Michael C. Sukop, Major Professor

Date of Defense: June 11, 2008

The dissertation of Shadab Anwar is approved.

$\begin{array}{r}\begin{array}{r}\text { Dean Kenneth Furton } \\ \text { College of Arts and Sciences }\end{array} \\ \hline \begin{array}{r}\text { Dean George Walker } \\ \text { University Graduate School }\end{array}\end{array}$

Florida International University, 2008 


\section{ACKNOWLEDGMENTS}

I wish to thank the members of my committee Dr. René Price, Dr. Dean Whitman and Dr. Yong Tao for their support and patience. Without their help this dissertation would not be possible. I especially thank my major advisor, Dr. Michael Sukop, for having eternal patience and providing the careful guidance needed to make my learning process come to fruition in the form of this dissertation. I am grateful to my advisor Dr. Michael Sukop and Dr. Danny Thorne for providing me their code LB2D_Prime. I am grateful to office staff at Department of Earth Sciences and CASTIC team for continuous support.

I would like to express my gratitude to the University Graduate School for the generous Dissertation Year Fellowship award during the last year of my dissertation. I am also thankful to the Graduate Students Association, FIU for providing me travel support to participate in international conferences.

I thank all those whom I have worked with in the Earth Sciences Department at FIU. I would like to thank my parents, family and friends for their continuous support. 


\section{DEDICATION}

To my beloved parents

Shakeela and Ainul 


\begin{abstract}
OF THE DISSERTATION
LATTICE BOLTZMANN MODELING OF FLUID FLOW AND SOLUTE
\end{abstract}

TRANSPORT IN KARST AQUIFERS

by

Shadab Anwar

Florida International University, 2008

Miami, Florida

Professor Michael C. Sukop, Major Professor

A novel modeling approach is applied to karst hydrology. Long-standing

problems in karst hydrology and solute transport are addressed using Lattice Boltzmann

methods (LBMs). These methods contrast with other modeling approaches that have been applied to karst hydrology. The motivation of this dissertation is to develop new computational models for solving ground water hydraulics and transport problems in karst aquifers, which are widespread around the globe. This research tests the viability of the LBM as a robust alternative numerical technique for solving large-scale hydrological problems. The LB models applied in this research are briefly reviewed and there is a discussion of implementation issues. The dissertation focuses on testing the LB models.

The LBM is tested for two different types of inlet boundary conditions for solute transport in finite and effectively semi-infinite domains. The LBM solutions are verified against analytical solutions. Zero-diffusion transport and Taylor dispersion in slits are also simulated and compared against analytical solutions. These results demonstrate the LBM's flexibility as a solute transport solver. 
The LBM is applied to simulate solute transport and fluid flow in porous media traversed by larger conduits. A LBM-based macroscopic flow solver (Darcy's law-based) is linked with an anisotropic dispersion solver. Spatial breakthrough curves in one and two dimensions are fitted against the available analytical solutions. This provides a steady flow model with capabilities routinely found in ground water flow and transport models (e.g., the combination of MODFLOW and MT3D). However the new LBM-based model retains the ability to solve inertial flows that are characteristic of karst aquifer conduits.

Transient flows in a confined aquifer are solved using two different LBM approaches. The analogy between Fick's second law (diffusion equation) and the transient ground water flow equation is used to solve the transient head distribution. An altered-velocity flow solver with source/sink term is applied to simulate a drawdown curve. Hydraulic parameters like transmissivity and storage coefficient are linked with LB parameters. These capabilities complete the LBM's effective treatment of the types of processes that are simulated by standard ground water models. The LB model is verified against field data for drawdown in a confined aquifer. 


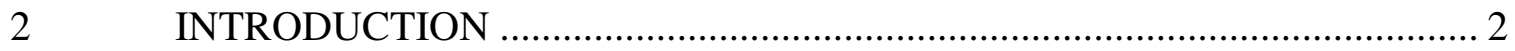

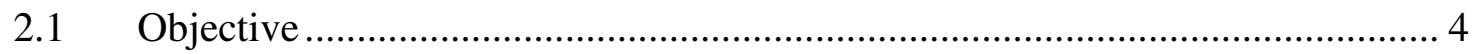

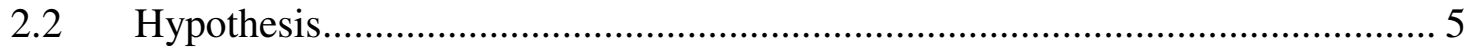

$2.3 \quad$ Karst aquifers ....................................................................................... 5

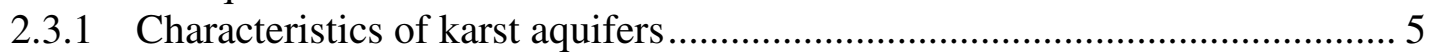

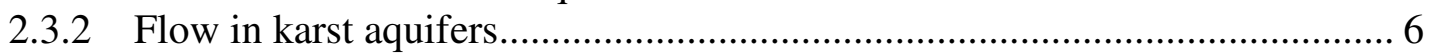

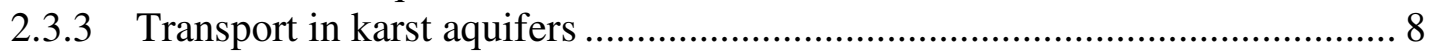

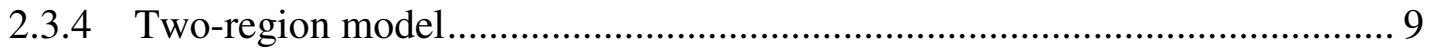

2.3.5 Breakthrough curves in karst aquifers .................................................... 10

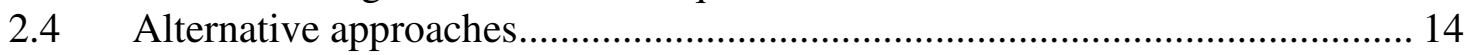

$2.5 \quad$ LBM for solute transport ..................................................................... 18

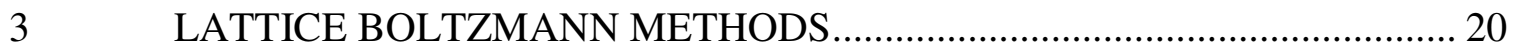

3.1 Navier-Stokes Bhatnagar-Gross-Krook model ............................................... 24

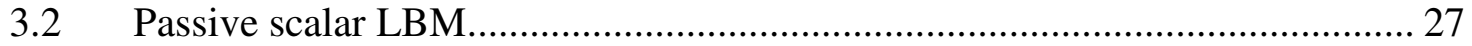

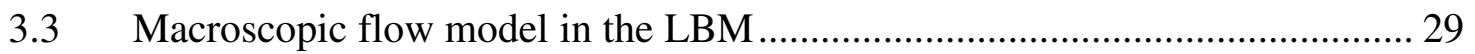

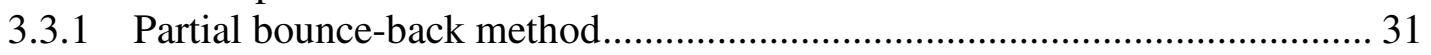

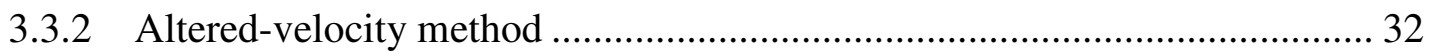

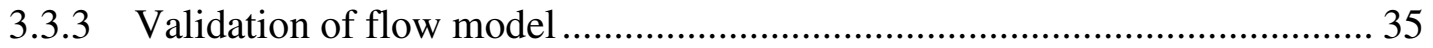

3.4 Anisotropic dispersion solver in the LBM................................................... 40

3.4.1 One-dimensional solute transport with anisotropic dispersion model........... 43

3.4.2 Two-dimensional solute transport with anisotropic dispersion ..................... 44

3.4.3 Two-dimensional solute transport with anisotropic dispersion for

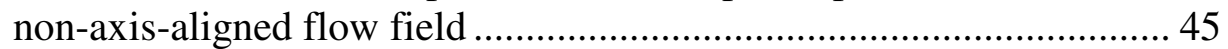

3.5 LBM on non-uniform grids .................................................................... 46

4 BOUNDARY VALUE PROBLEMS IN SOLUTE TRANSPORT ….............. 48

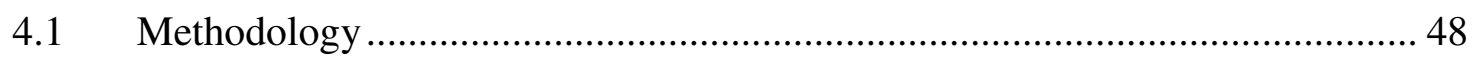

4.2 Zero-diffusion transport ................................................................................. 49

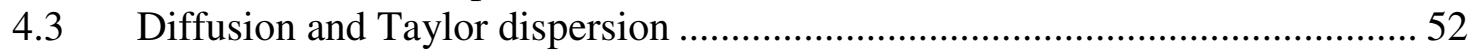

4.4 Concentration injection and detection modes ............................................... 55

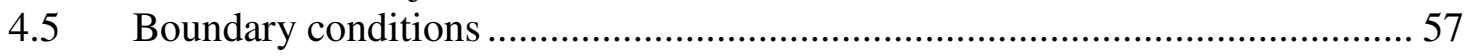

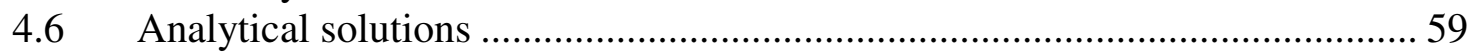

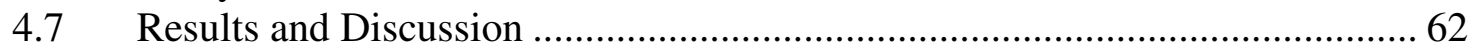

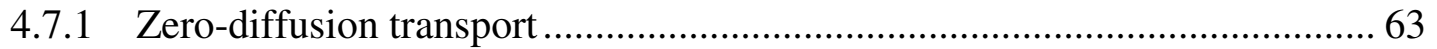

4.7.2 One-dimensional Advection-Diffusion Problem .............................................. 65

4.7.3 Taylor-dispersion problem........................................................................ 66

5 SOLUTE TRANSPORT IN KARST AQUIFERS …………………….......... 70

5.1 Example applications to karst flow and transport processes ........................... 75 
$5.2 \quad$ Two-region solute transport model ...................................................... 75

5.3 Transport in heterogeneous porous media .................................................. 84

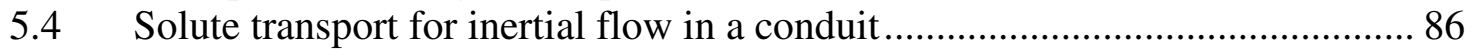

6 TRANSIENT GROUNDWATER FLOW SIMULATION IN CONFINED

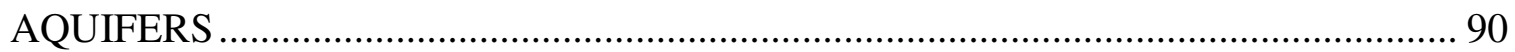

6.1 Transient groundwater flow equations.................................................. 92

6.2 Boundary value problem in a confined aquifer without source/sink ............... 93

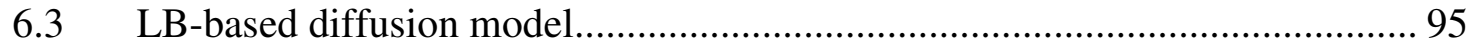

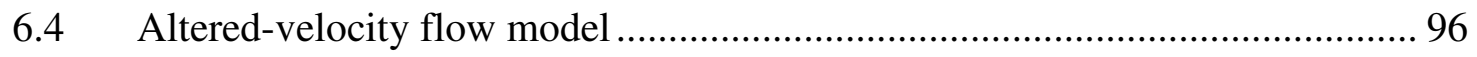

6.5 Source/Sink in groundwater flow model .................................................... 98

6.6 Transient ground water flow problem with source/sink .............................. 103

6.7 Validation of LB model with field data for drawdown data in a confined

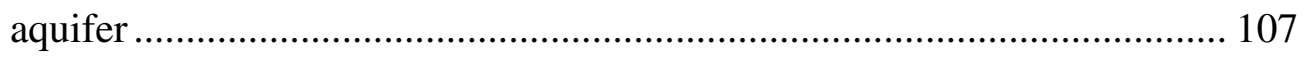

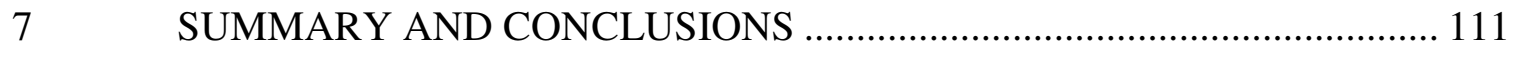

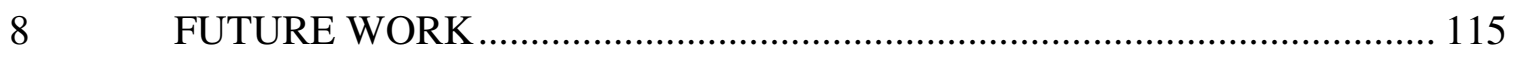

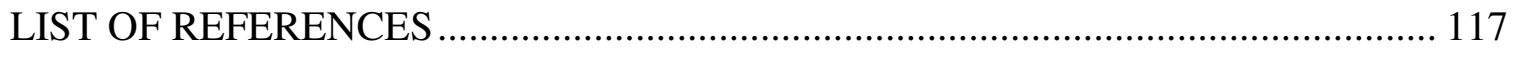

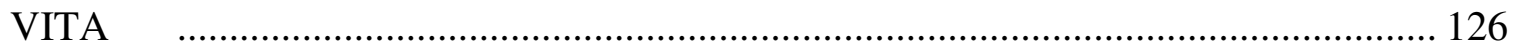




\section{LIST OF FIGURES}

FIGURE

PAGE

Figure 1. Effluent breakthrough from modified dataset from Superfund site in Tennessee (Field, 1999). 11

Figure 2. Breakthrough curves from Stuttgart tracer test (modified after

Goldscheider, 2008).

Figure 3. Tracer (Rhodamine dye) breakthrough curve observed in the Northwest Well Field (NWWF) in Miami-Dade County, Florida (Renken et al., 2008).

Figure 4. Laboratory experimental data for breakthrough curves of benzene on MCM-48 (Shim et al. 2005).

Figure 5. Lattice structure showing nodes from which groups of particles will stream in discrete directions (1-8) at discrete velocities $e_{j}$. D2Q9 refers to a 2dimensional model with 9 discrete particle groups

Figure 6. Speed distribution for molecules at equilibrium at different temperatures calculated using the Maxwell-Boltzmann theory.

Figure 7. Histogram showing direction-dependent particle distribution function for D2Q9 LBM.

Figure 8. Discrete fractions of molecules shown with their corresponding discrete

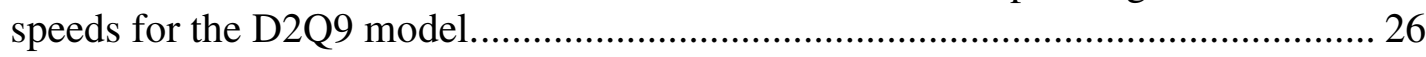

Figure 9. Flux is plotted against the pressure difference across the domain.

Deviation from Darcy's Law grows significantly as pressure difference and flux increase.

Figure 10. Poiseuille flow profile between the parallel plates using altered-velocity flow model. 38

Figure 11. Flow profile between parallel plates filled with porous media of $\mathrm{R}=1 \mathrm{ts} \mathrm{s}^{-1} \ldots . .40$

Figure 12. Spatial breakthrough curve for solute transport in one-dimensional flow field with uniform dispersivity $1 l u$.

Figure 13. Solute plume for $u=0.01 l u / t s$ and $\mathrm{D}_{\mathrm{L}}=1 l u \mathrm{D}_{\mathrm{T}}=0.1 l u$ in a $200 l u \times 20$ $l u$ domain after $5000 t s$. Domain is periodic in vertical direction. Solute was introduced at $(10,0)$ at time $=0$.

Figure 14. Spatial breakthrough curve for $D_{L}=1 \mathrm{lu}$ and $D_{T}=0.1 \mathrm{lu}$ in a $200 l u \times 20$ lu domain. 
Figure 15. Contour map of solute concentration in flow field unaligned with the LBM coordinates $\left(u_{x}=0.01 \mathrm{lu} / \mathrm{ts}\right.$ and $\left.u_{y}=0.005 \mathrm{lu} / \mathrm{ts}\right)$ with $D_{L}=0.5 \mathrm{lu}$ and $D_{T}$ $=0.1 \mathrm{lu}$. The LBM solution is shown in red and analytical solution is shown in green for comparison.

Figure 16. Parabolic velocity profile in laminar flow. 51

Figure 17. Solute front for zero diffusion with uniform flow in a parallel slit (1000 $l u \times 80 l u)$.

Figure 18. Breakthrough curve for zero diffusion in a slit at distance $L=100 l u$ from inlet. The slit width $2 a$ is $80 l u$, the average velocity is $0.02 l u-t s^{-1}$ the kinematic viscosity is $0.1666 l u^{2} t s^{-1}$ and the simulation's diffusion coefficient is $0.000333 l u^{2} t s^{-1}$, leading to a Peclet number $P e=6667(P e \rightarrow \infty)$.

Figure 19. Breakthrough curves for four different set of inlet and outlet boundary conditions at $P e=1 . \mathrm{C}_{\mathrm{f}}$ is flux averaged concentration and $\mathrm{C}_{\mathrm{r}}$ is resident concentration. A1, A2, A3, and A4 are defined in Table 1.

Figure 20. Breakthrough curve at $P e=1$ for transport in parallel slit for four different combination of inlet boundary conditions and domain type.

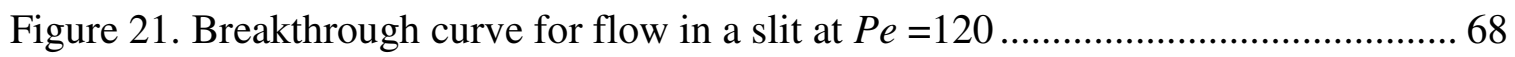

Figure 22. Schematic diagram of two-region model. ............................................... 78

Figure 23. LB simulation fitted with two-region model and advection-dispersion (ADE) model for $\beta=0.2$ and $\beta=0.4$.

Figure 24. Solute breakthrough curve measured at the end of $20 \times 40 l u$ domain with conduit (10lu) in the center. The breakthrough curve (open circles) obtained from the LBM is fitted with advection-dispersion equation (ADE) (solid line) and two-region (broken line) models

Figure 25. (a) Heterogeneous permeability field in grey scale. Lighter grey represents higher permeability. Red color is tracer and white is fluid (b) Snapshots of solute front before breakthrough and (c) during flushing of domain shown in (a). The units are in $l u$. 84

Figure 26. Effluent breakthrough for heterogeneous domain as shown in Figure 25...... 85

Figure 27. (a) Heterogeneous porous background with conduit. (b) Simulated snapshot of solute in domain soon after initial solute injection and (c) during flushing of domain. The units are in $l u$. 
Figure 28. Effluent breakthrough curve simulated with the LBM and fitted tworegion and advection-dispersion equation (ADE) for inertial flow conditions $(\operatorname{Re}=900)$ in domain of Figure 27. 88

Figure 29. Schematic representation of confined aquifer with boundary conditions 94

Figure 30. Transient change in head as a response to a sudden change in head at right boundary for two different solutions techniques vs. analytical results.

Figure $31.50 l u \times 10 l u$ domain to simulate 1-D flow with line source and sink nodes. Black lines at two ends represent solid boundaries (wall). The dark blue vertical line near the left end represents line of source nodes (density higher than neighboring nodes) and the white vertical line near the right end represents line of sink (density lower than neighboring nodes). A pressure gradient is created between source and sink due to imposed density difference....

Figure $32.51 l u \times 51 l u$ domain with source and sink nodes with porous media in background.

Figure 33. Quiver plot (velocity vector) with pressure contour lines for domain with walls on all four sides.

Figure 34. Quiver plot (velocity vector) with pressure contour lines for domain with fixed pressure boundary on all four sides.

Figure 35. Schematic representation of drawdown in a confined aquifer for a constant pumping rate $(\mathrm{Q})$......

Figure 36. Drawdown curve at observation well in a confined aquifer compared against the Theis solution.

Figure 37. Theis curve for constant hydraulic diffusivity $(D)$ and different storage coefficient $(S)$.

Figure 38. Validation of LB model against the field data for drawdown curve. 108

Figure 39. Field recovery data for an aquifer test in Biscayne aquifer. Well PB 1545 is located in West Palm Beach and well G3839 is located in Miami (Reese and Wacker, 2007).

Figure 40. Prototype karst aquifer with circular sinkhole (black) surrounded by porous media (grey). Pumping well is shown in blue and observation well is shown in red.....

Figure 41. Simulated time-series drawdown in prototype karst aquifer (Figure 40) using altered-velocity LBM. 
List of Symbols

\begin{tabular}{|c|c|}
\hline$\tau$ & Relaxation parameter $(t s)$ \\
\hline$\Delta t$ & Time steps $(\mathrm{T})$ \\
\hline$\xi$ & Diffusion length \\
\hline$\rho$ & Density $\left(\mathrm{ML}^{-3}\right)$ \\
\hline$\beta$ & Mobile fraction in two-region model \\
\hline$\omega$ & Non-dimensional mass exchange coefficient \\
\hline$\lambda$ & Mass exchange coefficient $\left(\mathrm{T}^{-1}\right)$ \\
\hline$\alpha_{\mathrm{L}}$ & Longitudinal dispersivity (L) \\
\hline$\alpha_{\mathrm{T}}$ & Transverse dispersivity (L) \\
\hline$\theta$ & Porosity \\
\hline$\Delta x, \Delta y$ & Grid spacing $(\mathrm{L})$ in $\mathrm{x}$ and $\mathrm{y}$ direction \\
\hline$v$ & Kinematic viscosity $\left(\mathrm{L}^{2} \mathrm{~T}^{-1}\right)$ \\
\hline$a$ & Half the width (L) of slit \\
\hline$A$ & Cross-sectional area $\left(\mathrm{L}^{2}\right)$ \\
\hline$b$ & Saturated thickness of confined aquifer (L) \\
\hline$c$ & Grid velocity $\left(\mathrm{LT}^{-1}\right)$ \\
\hline$c_{\mathrm{s}}$ & Speed of sound $\left(\mathrm{LT}^{-1}\right)$ \\
\hline$C$ & Concentration $\left(\mathrm{ML}^{-3}\right)$ \\
\hline$C_{0}$ & Reference concentration $\left(\mathrm{ML}^{-3}\right)$ \\
\hline$C_{\mathrm{r}}$ & Resident concentration $\left(\mathrm{ML}^{-3}\right)$ \\
\hline$C_{\mathrm{f}}$ & Flux concentration $\left(\mathrm{ML}^{-3}\right)$ \\
\hline
\end{tabular}




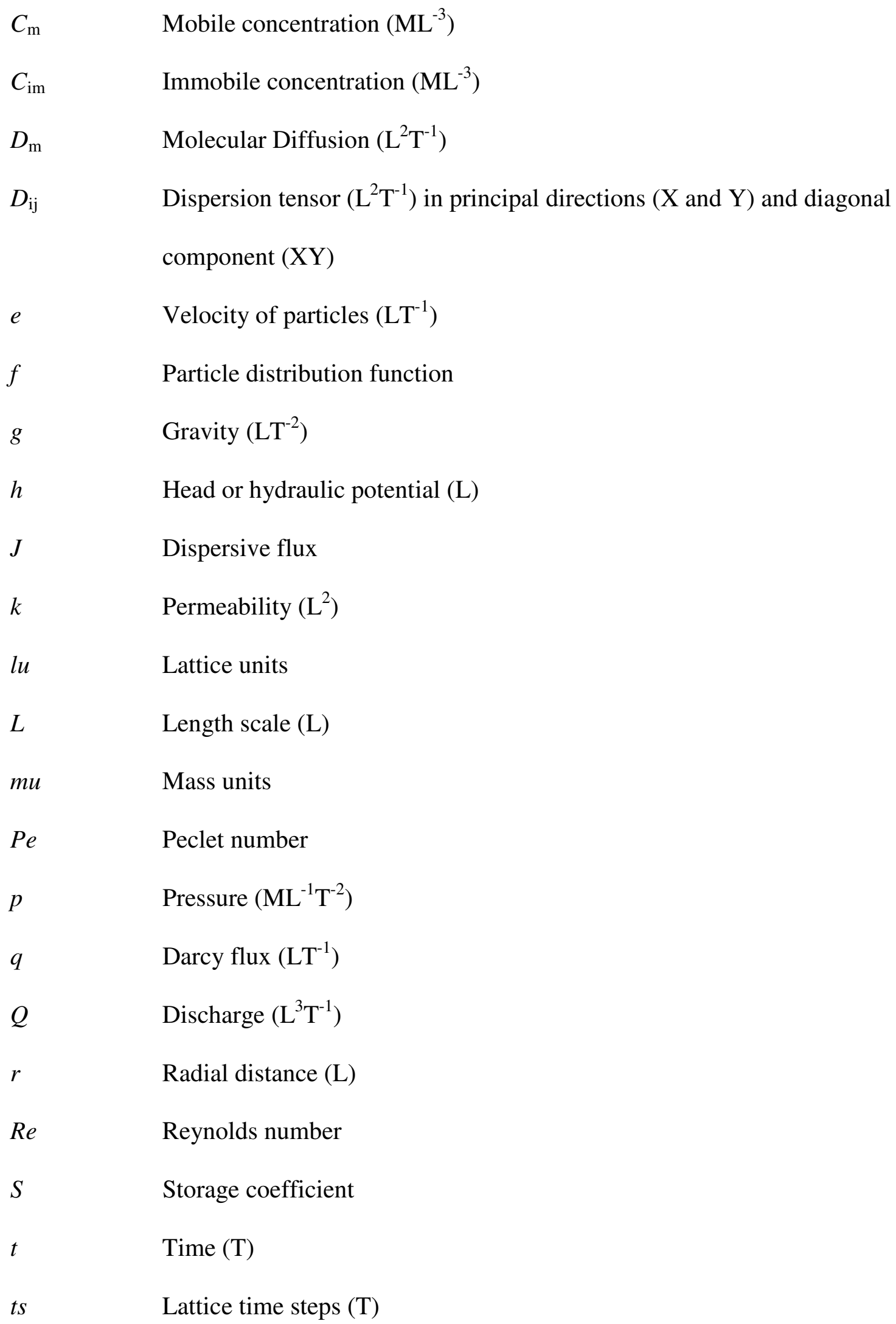


$T$

$u$

W
Pore volume

Average flow velocity $\left(\mathrm{LT}^{-1}\right)$

Width of confined aquifer (L) 


\section{PREFACE}

The work described in this dissertation was carried out between September 2004 and April 2008 at the Department of Earth Sciences, Florida International University, Miami. The study has been funded by the National Science Foundation (NSF), University Graduate School, and the US Geological Survey. As described in the abstract, in this dissertation the LBM is established as an alternative, robust numerical scheme for flow and solute transport in karst aquifers. The dissertation consists of six chapters. An introduction in the first two chapters provides much of the general background about solute transport and the LBM. Three chapters are based on papers that are accepted by or intended for publication in referred journals. Chapter 6 is to appear in Progress in Computational Fluid Dynamics and Chapter 5 is accepted for publication in Ground Water. A small portion of Chapter 5 is published in Progress in Computational Fluid Dynamics. Chapter 4 is intended for future submission. There is a final summary and conclusion which summarizes the essence of the dissertation. 


\section{INTRODUCTION}

The movement of dissolved chemicals in the environment is called solute transport. This can include transport in rivers and streams, in the unsaturated zone, and in saturated ground water aquifers. Various human activities discharge chemical and biological waste to land and water bodies, and the waste can percolate down to ground water leading to contamination of ground water resources. Such contamination may spread over long distances.

Important factors that characterize ground water contamination are (Schwartz and Zhang, 2003):

- degree of localization (point, non-point source, etc.),

- loading history (continuous, pulse, etc), and

- kinds of contaminant present (miscible solute, non-aqueous phase liquid (NAPL), radioactive, etc. ).

When a contaminant originates from a single location, it is called point source contamination. Non-point source contamination is a widespread threat to natural resources because it originates from many diffuse sources, mainly runoff from farmland or urban areas. Contamination manifests in both surface waters and ground water depending upon catchments and geological characteristics. Better understanding of contaminant transport in surface and ground water is critical for remediation and conservation of large-scale aquifers. 
Numerous analytical and numerical approaches have been used to simulate solute transport and the results depend upon the applied initial and boundary conditions. The applied boundary and initial conditions must correspond to the field or laboratory situation in order to obtain good simulation results (Kreft and Zuber, 1978). Several studies have evaluated the selection of initial and boundary conditions for onedimensional laboratory columns (James and Rubin, 1972; Parker, 1984; Novakowski, 1992). Proper formulation of the boundary conditions becomes critically important when analyzing laboratory displacement experiments involving relatively short columns, as well as for interpreting tracer data from laboratory or field profiles exhibiting large dispersivities (van Genuchten and Wierenga, 1986). Van Genuchten and Wierenga (1986) compiled analytical solutions of the advection-dispersion equation (ADE) for different boundary conditions in finite and semi-infinite domains (Table 1).

The breakthrough behavior of solute (time dependent concentration at a receptor is called a breakthrough curve) can determine the intensity and duration of exposure to a chemical, which in turn can determine its potential effects on humans in particular or ecosystems in general. Breakthrough behavior is a result of the complex interplay between diffusion and advection, which is controlled by head gradients and the fundamental pore scale of a system, which can range from microscopic pores to conduits on the order of 10s of meters. In porous media for example, pores which are not interconnected form dead end pore regions which trap contaminants and release them by means of diffusion over a long time. Similarly, contaminants that become trapped in eddies can only escape via diffusion and this may play a significant role in the flushing of 
any river or karst aquifer system. These processes, which are not well treated in traditional solute transport modeling, represent an area of opportunity for Lattice Boltzmann Methods.

\subsection{Objective}

The objectives of this research are to develop and validate lattice Boltzmann models as an alternative for flow and solute transport modeling in porous media with conduits and caves. A new Lattice Boltzmann model that incorporates a Darcy's law ground water flow solver and an anisotropic solute transport solver, while retaining the ability to simulate inertial flows (high $R e$ ) and solute transport in conduits, is developed. The model is used to explore various types of flow and solute transport problems in porous media and the effect of inertial flow conditions with eddy mixing on solute transport. This model will also consider contributions from dead-end regions that give rise to long-tailed breakthrough curves. The model is also verified for transient ground water flow problems in confined aquifers with sources and sinks to simulate physical situations that arise in karst aquifers with sinkholes, springs, and wells. This dissertation will show several verifications of LBM-based flow and transport models against the relevant analytical solutions. In Chapter 3, verification of the macroscopic flow and transport models has already been shown in the literature, and the purpose of such reverification is to test the particular code developed for this dissertation. Model verifications described in Chapters 4, 5 and 6 have not been presented previously elsewhere. This dissertation is focused on testing the suitability of the LBM as an alternative tool for modeling flow and transport in karst aquifers; hence the research 
emphasizes linking LBM models described in the literature (although new computer code had to be written and tested to implement and link these models) and not the development of new LBM algorithms. This research is believed to be the first attempt to link three different LBM models to simulate the processes of inertial and Darcy flow, and solute transport involving eddy mixing, anisotropic dispersion, and matrix diffusion in karst aquifers.

\subsection{Hypothesis}

A model for simulation of complex flow and transport process characteristic of karst aquifers can be constructed by joining the Darcy's law and anisotropic advectiondispersion equation (AADE) solvers with the normal Navier-Stokes capabilities of the LBM. This hypothesis will be tested against appropriate available analytical solutions and laboratory or field data.

\subsection{Karst aquifers}

\subsubsection{Characteristics of karst aquifers}

Karst aquifers (water bearing rocks) are formed by the dissolution of carbonate rock due to water-rock interactions. Karst evolves through dissolution of original discontinuities (fractures, joints, bedding planes) in limestone and dolomite "matrix" rocks and can become organized into a hierarchical, river-like system. Dissolution of rock causes the formation of large open zones (caves and conduits) and large vuggy pores. The rock matrix can be characterized as a traditional porous medium. Fractures, springs, sink and swallow holes are also likely to be present (White, 2002). 
Epikarst is the exposed surface region of any karst rock which is in contact with soil where intense chemical weathering can occur and large fissures and fracture enlargement can develop (Drew, 1995). Carbon dioxide from the atmosphere and in the soil combines with infiltrating water to create carbonic acid, which dissolves the rock. Beside climatic and biological conditions, the nature of preexisting porosity, the type of recharge, and the magnitude and direction of hydraulic gradient are important factors which determine conduit or cave patterns at local and regional scales (Palmer, 2000). The foremost characteristic that makes karst different from other terrains is its dynamic nature, both hydrologically and geologically.

\subsubsection{Flow in karst aquifers}

Karst aquifers can have strongly varying permeability which is a major factor in controlling the nature of flow in them. The variation in potentiometric surface in a karst aquifer often reflects the variation in permeability. The potentiometric surface does not remain smooth when conduit flow becomes more dominant (Smart and Ford, 1986).

Surface water and ground water are often hydraulically connected in a karst aquifer. A surface stream may disappear if the karst aquifer lying underneath is permeable or they may flow directly into sink or swallow holes. Streams can gain water if the karst aquifer's water table lies above the surface water stage. Karst aquifers help in preventing flooding in high precipitation regions by rapidly draining surface streams underground. Subsurface streams generally reappear elsewhere in the form of springs (White, 2002). 
Flow in karst takes place through anisotropic pathways (White, 2002) and can be explained in terms of conduit and diffuse flow (Field, 1993). A karst system is generally composed of porous matrix, fractures, and highly permeable conduit regions. Flow patterns are usually assumed to be diffuse (Darcian) in the porous matrix, laminar in fractures, and turbulent in conduits. The conduits and fractures are tortuous and head gradients may be high, hence the flow may become turbulent.

The Reynolds number $(R e)$ is a dimensionless number that indicates the relative balance between inertial and viscous forces and can serve as a measure of the likelihood of turbulent flow. Reynolds number is calculated as (Bear, 1972)

$$
\operatorname{Re}=\frac{u L}{v}
$$

where $u$ is the average velocity of fluid $\left(L T^{1}\right), \mathrm{L}$ is a characteristic length $(L)$, and $v$ is the kinematic viscosity $\left(L^{2} T^{1}\right)$ of fluid.

Widths of karst conduits (characteristic length) could be several meters and sometimes they have flow like a free-surface, turbulent river at very high flow rate. For example, Florida's largest spring (Wakulla spring near Tallahassee) has an opening approximately $100 \mathrm{~m}$ across and a discharge of $\sim 10 \mathrm{~m}^{3} / \mathrm{s}$ (Scott et al. 2002). The corresponding Reynolds number is approximately 10 million. Flow in fractures becomes turbulent when the Reynolds number exceeds 500 (White, 2002), although eddies can form at much lower Reynolds number and exert a strong influence on solute transport. When flow reaches the eddy and turbulent regimes the relationship between head loss 
and discharge becomes non-linear in contrast to the relationship expressed by Darcy's law.

Conduit flow has been described with the Hagen-Poiseuille equation for laminar flow or with the Darcy-Weisbach equation in turbulent regimes (Howard and Groves, 1995; White and White 2005; Birk et. al., 2004; Shoemaker et. al., 2008; Diersch, 2002). In the Darcy-Weisbach equation, flow resistance is specified by an empirical friction factor which depends upon the Reynolds number in a complex way. White and White (2005) showed that the friction factor back-calculated using the Darcy-Weisbach equation differs from the friction factor determined from roughness or the Reynolds number or both, depending upon the flow regime. This discrepancy shows that the DarcyWeisbach equation is not reliable to describe turbulent flow in conduits.

Models of flow in karst systems have generally used analytical expressions that depend upon empirical parameters or are founded on Darcy's Law. Darcy's law is applicable for flow in laminar regime only and does not account for inertial effect which can occur in conduit or macropores generally found in karst aquifers. Therefore, a better model is needed to study karst hydrology.

\subsubsection{Transport in karst aquifers}

Study of solute transport is important for environmental protection and remediation. Chemical contamination may enter karst systems through sinkholes or caves and attenuate in a short time or it can get trapped in the epikarstic zone and eventually be released into the aquifer (Field, 1993). Different methods have been developed to 
understand the subsurface transport of solute in different geological media. Traditional ground water flow and solute transport modeling are based on Darcy's law and the anisotropic advection-dispersion equation respectively, and are principally applicable to porous media like sand. The nature of solute transport in karst systems suggests that they have two interrelated features that differentiate them from typical porous media aquifers. First, large openings constitute preferential flow paths that interact with the surrounding matrix in a complex way. Second, the porous matrix surrounding the preferential flow paths has relatively low permeability and interacts with both the flow and transport in the preferential paths. Two-region models are an initial mathematical approach to simulating this process. Large openings can cause flows at high Reynolds number, which develops eddy mixing of solutes with its corresponding complexities.

\subsubsection{Two-region model}

The two-region model is a conceptual model consisting of two domains: a "mobile" domain in which transport is solved by advection and dispersion, and an "immobile" domain in which flow is assumed negligible. Basically, a source/sink term is added in the advection-dispersion equation to link transport in the two flow regions. The transport between mobile (conduit) and immobile (matrix) regions is linked using first order mass exchange between the two flow regions. This gives a pair of equations that can be solved for mobile and immobile concentrations. Breakthrough curves obtained from this type of model for a relatively small mobile fraction show a sudden rising limb followed by slow rise towards full breakthrough. For input pulse durations shorter than 1 pore volume, there is a sudden drop before complete breakthrough, followed by tailing, which depends on the mass exchange rate between the two regions. This model is state- 
of-the-art for breakthrough curves obtained from karst aquifers. The drawbacks and limitations of this model for prediction of breakthrough curves from karst aquifers are shown in Chapter 5.

\subsubsection{Breakthrough curves in karst aquifers}

A significant number of environmental problems, including karst water supply and contamination problems, involve fluid flow and solute transport through sub-surface fracture/conduits zones. The presence of open fractures or conduits surrounding less permeable matrix create systems with advection-dominated flow in the fracture/conduit zone and diffusion/dispersion-controlled transport in the matrix zones. This can give rise to long-tailed, asymmetric breakthrough curves. The breakthrough curve of a chemical from such a medium generally has three characteristic features (as shown in Figure 1): sudden rising limb, plateau, and a long tail. The sudden rising limb is due to the conduit region and the long-tail is due to slow diffusion from the matrix region. There is an additional feature observed in breakthrough curves from karst aquifers that will be referred to as "intermediate plateaus". The presence and width of these plateaus depends on the pulse duration and relative permeability of the matrix and conduit regions. Intermediate plateaus may not be seen in a breakthrough curve for a pulse of extremely short duration. Intermediate plateaus can be approximated with a two-region-type model under certain conditions as described in Chapter 5. For longer pulse duration, multiple plateaus may exist depending on the number of discrete permeability regions in a karst aquifer. Such systems can produce breakthrough curves too complex to be simulated with a two-region-type model. 
As shown in the examples below, intermediate plateaus may be common or even universal in breakthrough curves from karst aquifers with conduits and significant matrix effects. The breakthrough curve shown in Figure 1 was obtained from a Superfund site in Tennessee (Field, 1999). It has a single intermediate plateau on the falling limb.

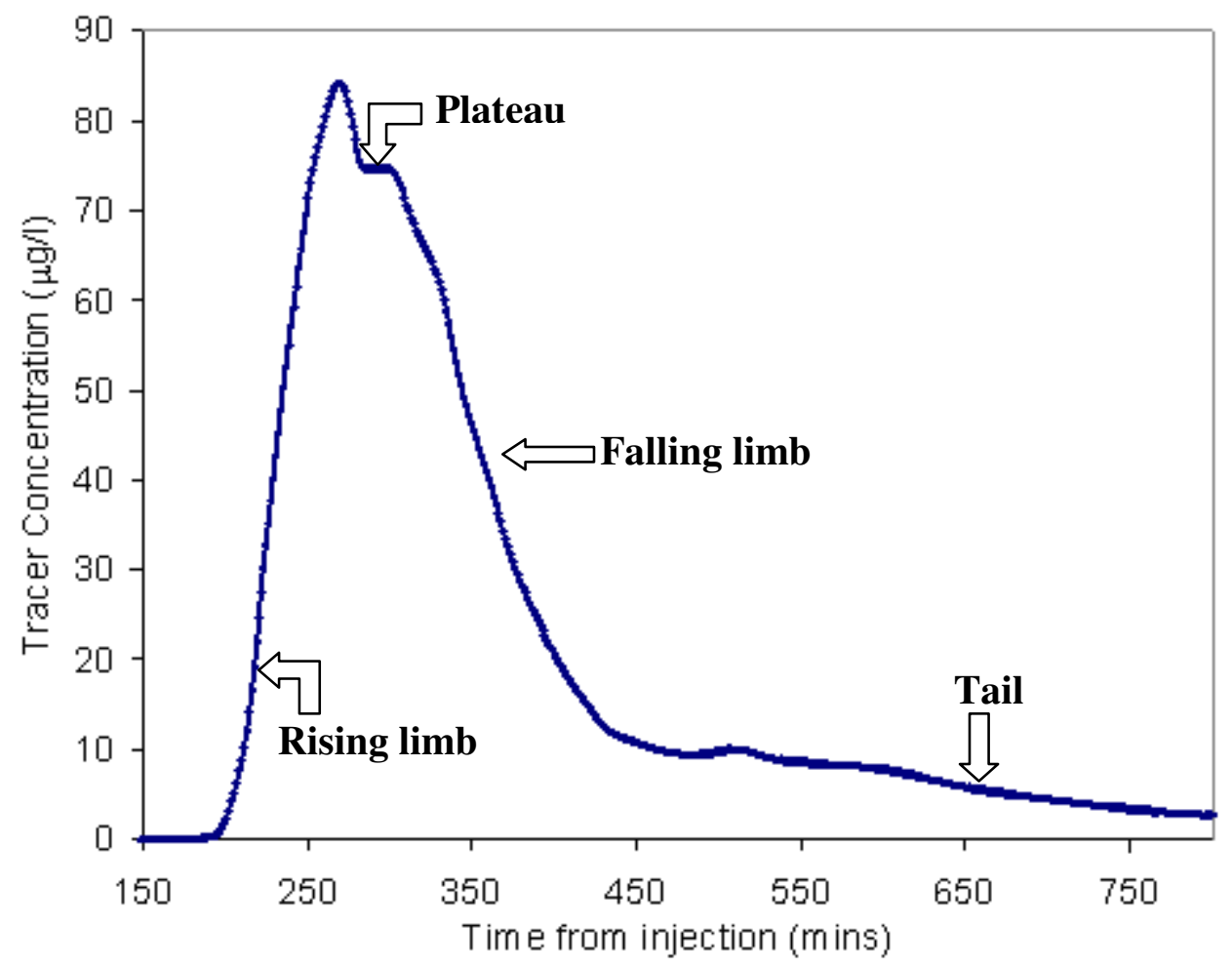

Figure 1. Effluent breakthrough from modified dataset from Superfund site in Tennessee (Field, 1999).

Figure 2 shows breakthrough curves from a tracer test in the city of Stuttgart, Germany (Goldscheider, 2008). The maximum flow velocity was found to be 53-104 $\mathrm{m} /$ day and the average flow velocity was 31-49 $\mathrm{m} /$ day. Possible intermediate plateaus can be seen on the rising and falling limbs. Such features are observed in LBM simulations as described in Chapter 5. 


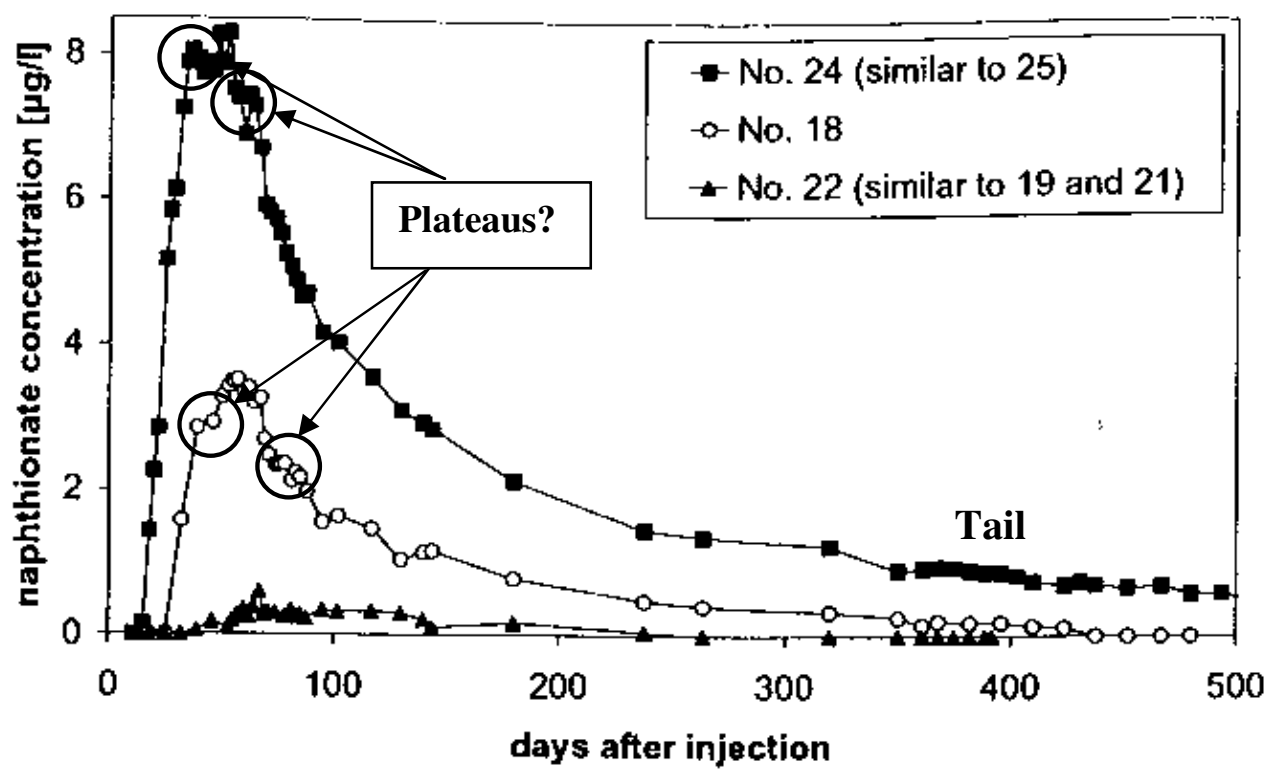

Figure 2. Breakthrough curves from Stuttgart tracer test (modified after Goldscheider, 2008).

An aquifer and tracer test were conducted in the Northwest Well Field (NWWF) in Miami-Dade County, Florida (Renken et al, 2008). The NWWF is Florida's largest well field and supplies water from the Biscayne aquifer to over two-million residents of south-Florida. The breakthrough curve obtained from the tracer test is shown in Figure 3. It shows typical karst aquifer breakthrough features—rapid initial rise, followed by short intermediate plateaus near the peak and during the falling limb. The large fraction of the injected mass recovered from the tracer experiment indicates that advection through conduits and large vugs is the dominant means of transport in the Biscayne aquifer (Renken et al, 2008). 


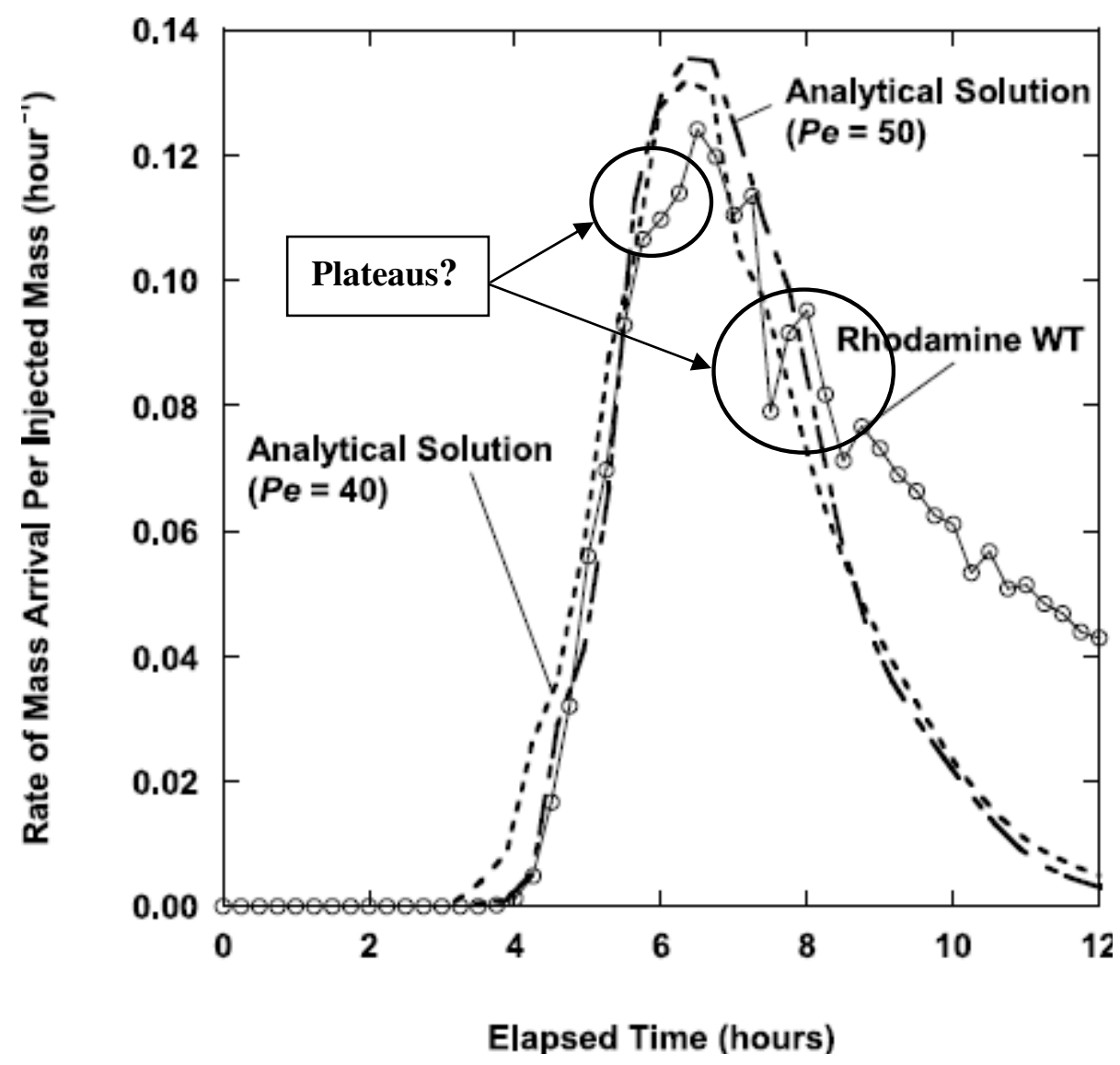

Figure 3. Tracer (Rhodamine dye) breakthrough curve observed in the Northwest Well Field (NWWF) in Miami-Dade County, Florida (Renken et al., 2008).

Figure 4 shows breakthrough curves for benzene through a packed column of pelletized MCM-48 adsorbent (MCM-48 is a large-pore molecular sieve material or adsorbent). It was found that the length of the intermediate plateau and time to reach complete breakthrough are highly sensitive to the inlet concentrations. The length of the plateau was observed to decrease by increasing the inlet concentrations (Shim et al, 2005). The observed breakthrough is fitted with a two-region type model with modified adsorption isotherm. 

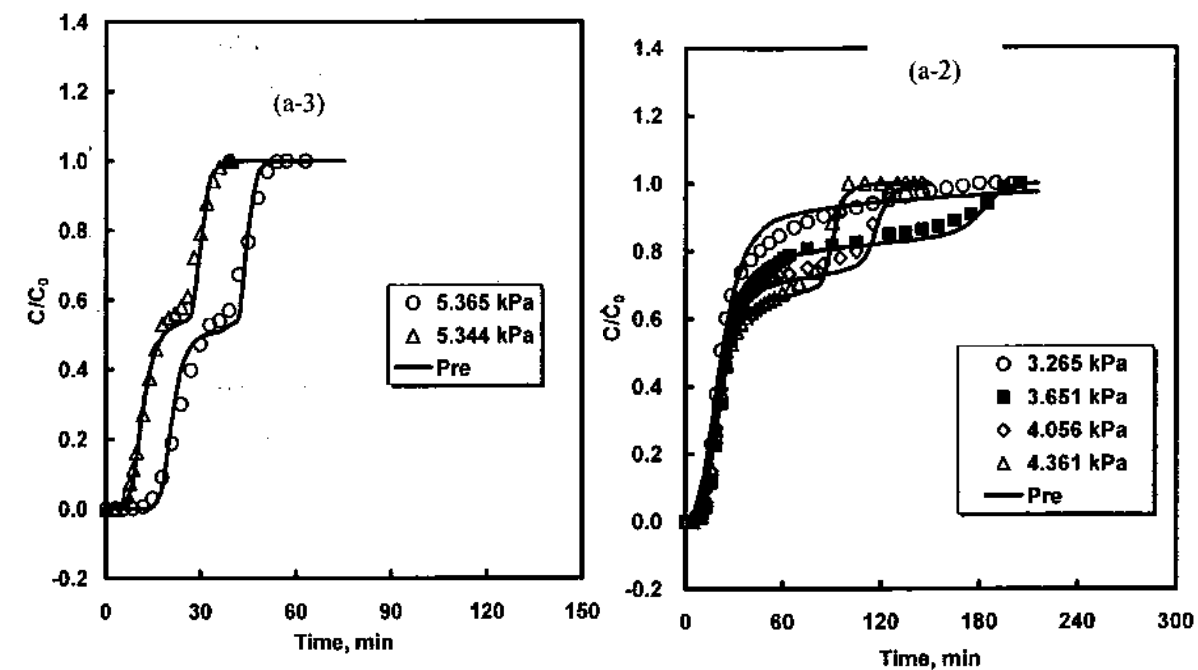

Figure 4. Laboratory experimental data for breakthrough curves of benzene on MCM-48 (Shim et al. 2005).

\subsection{Alternative approaches}

"Black box" (Empirical) and "distributed" models are the two fundamental approaches to modeling karst systems. In the black box model, different techniques like recession analysis (separate analysis of recession curve (tailing) as observed in storm hydrograph or breakthrough curve from karst aquifers), regression analysis, mixing cell models, and kernel/transfer functions are used to simulate karst systems. Some hydraulic parameters and a response function of karst systems can be estimated using such methods (Sauter, 1993). This method ignores the details of the various processes that determine flow and transport in a karst aquifer due to its lumped-parameter nature. The advantages of such methods are that data requirements are minimized and simulation is faster. 
Distributed parameter models were developed to utilize available information about variability of hydrological properties in karst aquifers. At least three methods have been used to describe flow and transport in karst systems with these models (CGER, 1996):

- Equivalent porous medium (EPM),

- Discrete fracture/conduit, and

- Multi-continuum and Hybrid approaches.

In a conventional equivalent continuum model or equivalent porous medium (EPM) approach, heterogeneity of the fracture network or matrix is modeled by assigning variable hydraulic conductivity to the domain and Darcy's Law is used over the whole continuum. Individual fractures or pores are not studied explicitly. The continuum approach represents the complicated spatial heterogeneity in a simple mathematical way that can easily be applied to numerous problems (CGER, 1996). At what scale the application of a continuum model is justified is still unknown (Cortis and Berkowitz, 2004).

A discrete fracture/conduit model does not consider the effect of the matrix on flow and transport and assumes flow is confined to the fracture/conduit regions (Barenblatt et al. 1960; Coats and Smith, 1964). Flow in fractures is assumed to behave as laminar flow between parallel plates (cubic law) and requires detail about fracture apertures, fracture length, orientation, and connectivity. These properties are often simulated stochastically because it is difficult to explicitly measure them. The main 
advantage of this model is that it considers the contribution of every fracture towards the overall transmissivity of aquifer and can be applied at any scale. The drawback of this model is that statistical information required for parameter estimation may also be difficult to obtain. This model may become very complex at field scale and computationally intensive too. Turbulent flows in conduits would typically be treated with the Darcy-Weisbach equation (CGER, 1996).

The multi-continuum approaches put these two above-mentioned approaches together to represent heterogeneity of karst aquifers (For example, Scanlon et al., 2003). One drawback of many of these models lies in the assumption that flow through the matrix and fractures is laminar and thus that Darcy's law and the cubic law are always applicable for flux calculation. Sharp and Maini (1972) showed a nonlinear flow regime in laboratory flow experiments in fractures. Similarly, DiFrenna (2005) and Alvarez (2007) found a non-linear relationship between flow and head gradient in karst rocks from south Florida.

Modeling ground water flow and solute transport is difficult in karst systems due to complicated geometry and complex interaction between diffusion- and advectiondominated regions. Some modifications to standard numerical models (e.g., MODFLOW) have been proposed in the past to more closely simulate karst conduit systems. In MODFLOW a continuous branching network of drain cells with drain elevation and conductance is used as an analogue to a karst conduit. The drain cell feature is available in MODFLOW to simulate agricultural drains that remove water from an 
aquifer at a rate proportional to the difference in water level and some fixed drain elevation (Quinn and Tomasko, 2000; Quinn et al., 2006). Sun and Painter (2004) linked a dual continuum model (DCM) to existing MODFLOW and proposed a new MODFLOW-DCM model to simulate turbulent flow in conduits and diffuse flow in the matrix. They have demonstrated the success of this model for ground water management in the Barton Springs segment of the Edwards aquifer in south-central Texas.

QTRACER is a computer program developed to analyse breakthrough curve data obtained from tracer studies in karst and fractured-rock aquifers (Field, 1999). Parameters such as the mass of the tracer, mean residence time, mean flow velocity, and longitudinal dispersion are estimated based upon the method of moments. Geometrical properties such as aquifer volume, cross-sectional area, karst conduit diameter etc., are estimated from discharge rate and mean residence time.

White (2002) discussed the widely-accepted triple permeability conceptual model, which includes matrix permeability, fracture permeability, and conduit permeability to simulate flow in karst aquifers. The flow field is calculated based upon Darcy's law in the matrix region, the cubic law in the fracture region, and the Darcy-Weisbach equation in the conduit region. White and White (2005) presented the relative contributions of each of the three permeability zones towards the overall ground water flux. This comparison justified the elimination of the least permeable zone during model development. A plot of discharge rate vs. aperture width on logarithmic scale for different hydraulic gradients, indicates that matrix flow is dominant in carbonate aquifers until fracture aperture widens 
due to dissolution and eventually conduit flow become dominant; however fracture flow dominates in an aquifer with low hydraulic gradient.

\subsection{LBM for solute transport}

Lattice Boltzmann methods are becoming established as reliable solute transport solvers (Flekkøy, 1993; Grubert, 1997; Stockman et al., 1998; Inamuro et al., 2002; Yoshini and Inamuro, 2003; Zhang et al. 2002 a, b; Ginzburg, 2005; Thorne and Sukop, 2004; Sukop and Thorne, 2006; Camas Serván, 2007; Anwar et al., 2008). In assessing the solute transport capabilities of the LBM, it is convenient to make use of the Peclet number $(\mathrm{Pe}) . \mathrm{Pe}$ is a dimensionless number that indicates the relative importance of diffusion (or dispersion) and advection in mass transport. It is a ratio between the time taken by fluid particles to traverse distance $L$ by diffusion alone $\left(t_{\text {diff }}\right)$ and the time taken to travel the same distance $L$ by advection $\left(t_{\text {adv }}\right)$ at average velocity $\mathbf{u}$ :

$$
P e=\frac{t_{\text {diffusion }}}{t_{\text {advection }}}=\frac{\frac{L^{2}}{D_{m}}}{\frac{L}{u}}=\frac{u L}{D_{m}} .
$$

Analytical solutions of the Advection-Dispersion Equation (ADE) under different boundary conditions are appreciably different from each other at small $P e$, hence the breakthrough curve obtained for different boundary conditions (as shown in Table 1) does not converge for small $\mathrm{Pe}$. This provides an opportunity for testing the LBM's solute transport simulation capabilities.

Lattice gas models preceded and are closely related to the LBM. Lattice gas models are based upon cellular automata and Boltzmann's kinetic theory of gases. Lattice 
gas models are built on the motion of discrete particles on regular lattices. Each lattice node has a Boolean variable $\left(n_{j}\right)$ to represent the presence or absence of a particle in each of a small number of possible lattice directions. The main development marking the transition from lattice gas to lattice Boltzmann models is consideration of the dynamics of a "group" of particles in discrete directions at every time by replacing the Boolean variable $n_{j}$ with a real-valued particle distribution function $f_{i}(\mathbf{x}, \mathrm{t})$. The basic mechanism of propagation and collision of particles at every time step (ts) remains unchanged.

Several researchers demonstrated the solute transport capabilities of lattice gasses. Baudet et al (1989) used a lattice gas model to simulate dispersion between parallel plates and the longitudinal dispersion coefficient matched very well with the Taylor-Aris model for Peclet numbers ranging from 4.3 to 35.4. Perea-Reeves and Stockman (1997) used a lattice gas model to simulate dispersion in alveolated channels (channels with pockets along the wall boundary). They considered how buoyancy affects the prediction of effective dispersion coefficients for a range of Peclet numbers. Stockman et al. (1998) demonstrated the ability of the LBM to successfully simulate dispersion and diffusive fingering in rough fractures. 


\section{LATTICE BOLTZMANN METHODS}

In this chapter, an introduction to the particle-based numerical scheme known as the Lattice Boltzmann method is presented. Three different LBMs are linked in this dissertation to simulate fluid flow and solute transport in porous media. The basic framework of the LBM is explained in the next section and subsequent sections describe the different models used. A passive scalar model is used to simulate solute transport in free-flowing fluid. Then a "macroscopic" or Darcy-scale model is described, which allows simulation of large-scale flows in porous media with LBMs. Finally, a LBMbased anisotropic dispersion solver, which is necessary to solve transport problems in macroscopic porous media, is incorporated. This model is verified against analytical solutions for flow and transport problems. All of these models are necessary to create a LBM simulator that incorporates all of the processes likely to be important in karst aquifers.

Lattice Boltzmann methods provide an alternative numerical method for simulating hydrodynamic systems governed by the Navier-Stokes equations for isothermal compressible fluid flow as shown below.

$$
\begin{gathered}
\frac{\partial \rho}{\partial t}+\nabla \cdot \rho \mathbf{u}=0 \\
\frac{\partial(\rho \mathbf{u})}{\partial t}+\nabla[\rho \mathbf{u u}]=-\nabla p+\nabla[\rho v(\nabla \mathbf{u}+\mathbf{u} \nabla)]
\end{gathered}
$$

Where $\rho$ is the density of fluid, $\mathbf{u}$ is velocity vector, $v$ is the kinematic viscosity of fluid, $p$ is pressure, $x, y$ are spatial coordinate, and $t$ is time. 
LBMs are based on discrete velocity, time, and lattice space (Figure 5) and are proven to recover the Navier-Stokes solution at macroscopic scale (Qian et al., 1992).

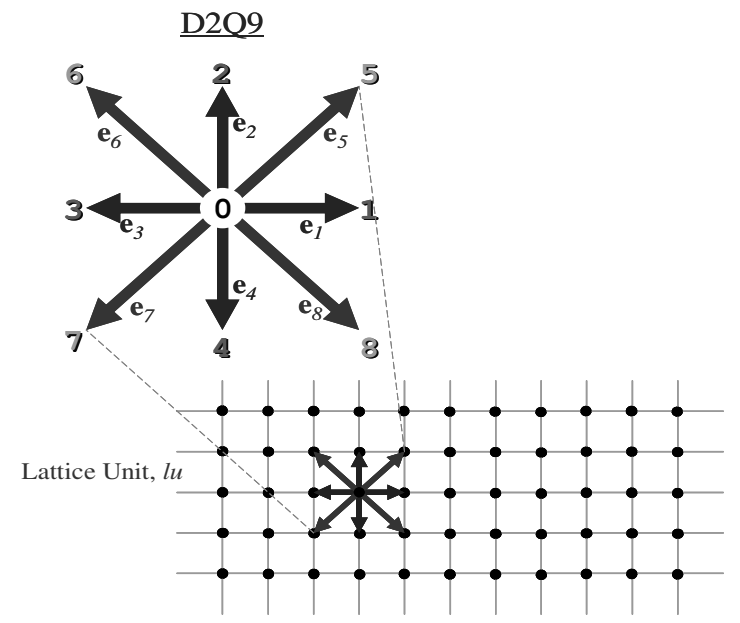

Figure 5. Lattice structure showing nodes from which groups of particles will stream in discrete directions (1-8) at discrete velocities $\mathrm{e}_{\mathrm{j}}$. D2Q9 refers to a 2-dimensional model with 9 discrete particle groups.

Unlike traditional numerical methods, the LBM does not discretize the governing equations at macroscopic scale in space and time; instead it solves the dynamics of hypothetical particles represented by particle distribution functions $\left(f_{\mathrm{j}}\right)$. The behavior of these particle distribution functions are governed by the Boltzmann equation. The Boltzmann equation governs the time rate of change of the particle distribution function. In simple words, this equation states that number of particles in a state is equal to the number of particles entering the state minus the number of particles leaving the state (Chen et al., 1994). The particle distribution function represents the dynamic state of a hypothetical group of particles in terms of its location and momentum at any time. 
In a system of $\mathrm{N}$ molecules where $\mathrm{N}$ is on the order of $10^{23}$, it is impossible to track the velocity of each and every molecule. The particles move around with different ranges of velocities, collide, and then their velocities change. The Maxwell-Boltzmann distribution theory states that particles conform to a speed distribution at equilibrium. The shape of the distribution is a function of temperature as shown in Figure 6.

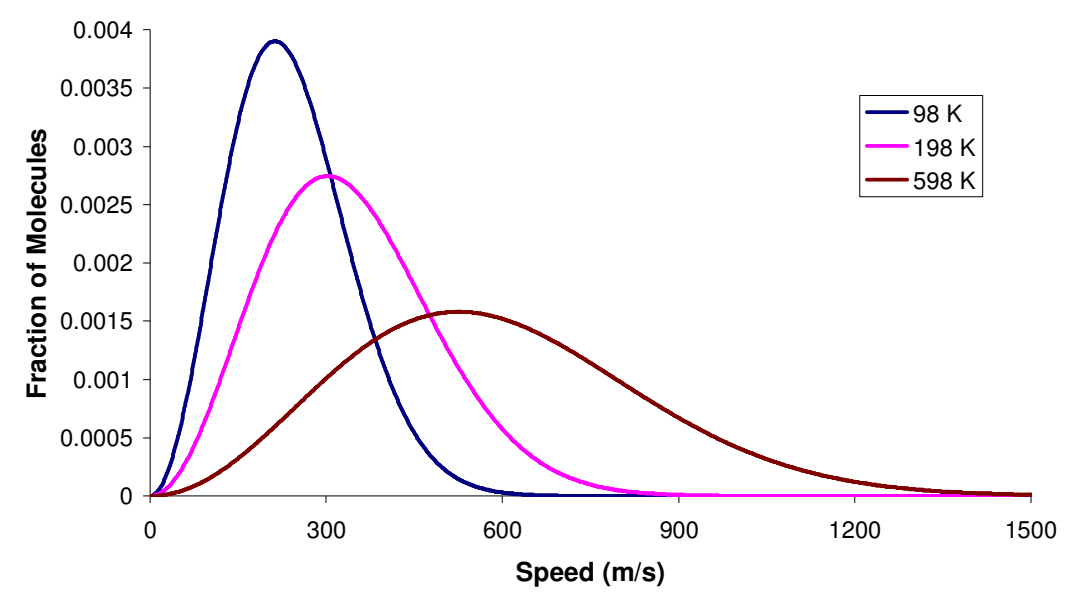

Figure 6. Speed distribution for molecules at equilibrium at different temperatures calculated using the Maxwell-Boltzmann theory.

The curves shown in Figure 6 are presented for three different temperatures. The area under the curve will always be equal to one, but the distribution of particle speeds will change with temperature.

Because of the particle basis of the LBM, there is no large set of simultaneous equations to solve as develops in conventional numerical methods (Ginzburg, 2005). Also, the LBM is amenable to parallel computation because most of the computational work is local and does not require data from remote nodes. 
The most commonly used classification of the $\mathrm{LBM}$ is $\mathrm{D} i \mathrm{Q} j$, where $i$ represents the number of dimensions $(1,2$, or 3 for 1,2 , or $3-\mathrm{D})$ and $j$ represents the number of discrete directions (3 for 1-D, 6 or 9 for 2-D and 15, 19, or 24 for 3-D) that are available for a group of particles (represented by the particle distribution function, $f_{j}$, as shown in Figure 7) to propagate. A D2Q9-type model is two-dimensional in space and has 9 discrete groups of particles at every node. Every group of particles is allowed to propagate to the nearest neighboring node with discrete velocity $\left(\mathbf{e}_{\mathrm{j}}\right)$. $\mathbf{e}_{\mathrm{j}}$ is equal to ratio between the distance between two nodes in lattice units $(l u)$ in the $j^{\text {th }}$ direction and lattice time step $(t s)$. There is also a group of particles which is immobile and considered to have discrete velocity $\mathbf{e}_{0}$ equals to zero.

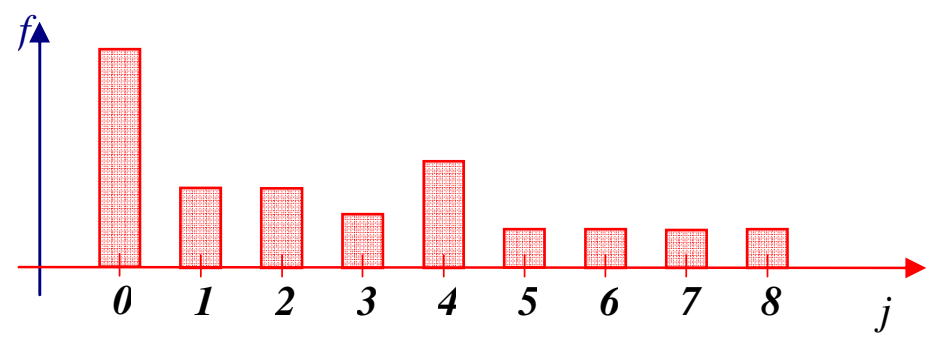

Figure 7. Histogram showing direction-dependent particle distribution function for D2Q9 LBM.

The macroscopic fluid density $(\rho)$ for the D2Q9 model is (Qian et. al., 1992)

$$
\rho=\sum_{j=0}^{8} f_{j} .
$$

The macroscopic velocity $\mathbf{u}$ is an average of the microscopic velocities $\mathbf{e}_{j}$ weighted by the directional densities $f_{j}$ :

$$
\mathbf{u}=\frac{1}{\rho} \sum_{j=0}^{8} f_{j} \cdot \mathbf{e}_{j} .
$$


Equations (5) and (6) link the particle distribution with the macroscopic density and velocity of the fluid.

Key steps in the LBM are streaming and collision of the particles via the distribution function. The simplest approach uses the BGK (Bhatnagar-Gross-Krook, 1954) approximation for collision as described below. Qian et al (1992) developed a local equilibrium function as shown below to recover the Navier-Stokes equations at macroscopic scale. Groups of particles represented by $f_{j}$ are allowed to stream to neighboring nodes in discrete directions (as shown in Figure 5) and undergo collision as described below.

\subsection{Navier-Stokes Bhatnagar-Gross-Krook model}

The simplest LBM approximately solves the Navier-Stokes (inertial) equations of fluid motion. Inertial flow is expected inside geometrically complex karst conduits at moderate flow velocity when the Reynolds number, which expresses the inertial 'content' of the flow as described later, is greater than approximately 1 . The ability to simulate such flows is a key advantage of using LBMs in karst aquifers.

The D2Q9 equilibrium distribution function $f^{e q}$ is (Qian et. al 1992)

$$
f_{j}^{e q}(x)=t_{j}^{*} \rho(\mathbf{x})\left[c_{s}^{2}+\mathbf{e}_{j} \cdot \mathbf{u}+\frac{3}{2}\left(\mathbf{e}_{j} \cdot \mathbf{u}\right)^{2}-\frac{1}{2} \mathbf{u}^{2}\right],
$$

where the weights $t_{j}^{*}$ are $1 / 3$ for $j=1,2,3,4$, and $1 / 12$ for $j=5,6,7,8$ and weight for rest particles $(j=0)$ is (Ginzburg, 2005) 


$$
t_{j}^{*}=1-c_{s}^{2} \sum_{j} t_{j}^{*}=1-\frac{5}{3} c_{s}^{2}=\frac{4}{9}
$$

The domain is discretized into a square space with lattice spacing $\Delta x=\Delta y=1 . c_{s}^{2}=\frac{1}{3} c^{2}$

where, $c_{s}$ is the speed of sound and $c$ is lattice speed, $c=\frac{\Delta x}{\Delta t}=1$ (Qian et. al., 1992). The speed of sound $\left(c_{s}\right)$ is a free parameter that can be changed to tune the model. Note that if $\mathbf{u}=0$, the equilibrium $f_{j}$ are simply the weights times the fluid density and sound speed squared.

Since the LBM is based upon the discrete Boltzmann equation, the velocity/particle distribution function $(f)$ is also discrete in nature. The entire set of molecules is distributed among three different speeds $(1, \sqrt{2}, 0)$. The fraction of particles with discrete speeds for the D2Q9 model is shown in Figure 8, which is a discrete analog of the continuous particle speed distribution function shown in Figure 6. Figure 8 represents the equilibrium distribution function $\left(f^{e q}\right)$ for the D2Q9 model. 


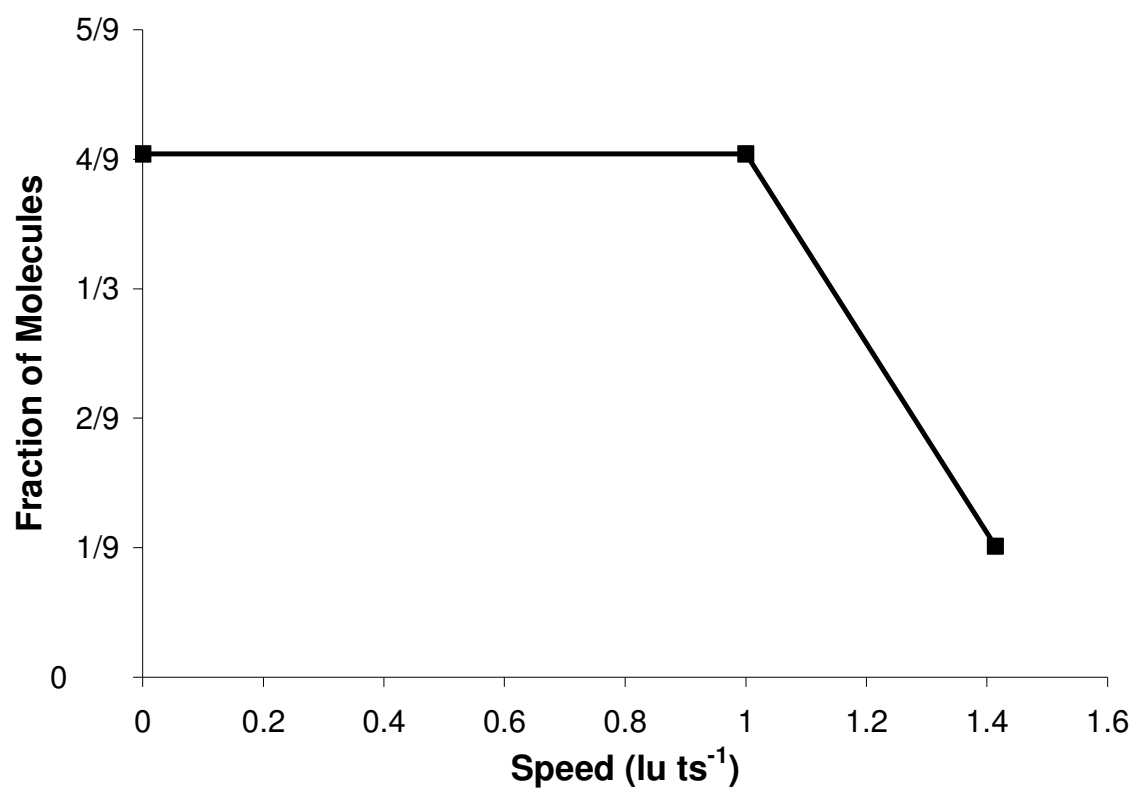

Figure 8. Discrete fractions of molecules shown with their corresponding discrete speeds for the D2Q9 model.

The LBM parameters have dimensions of mass units $(m u)$ for mass, lattice units $(l u)$ for space, and lattice time steps $(t s)$ for time. The similitude between lattice dimension and physical dimension is established using non-dimensional numbers as described in subsequent chapters.

Equation (8) represents the time evolution of the particle distribution function:

$$
\underbrace{f_{j}\left(\mathbf{x}+\mathbf{e}_{j} \Delta t, t+\Delta t\right)=f_{j}(\mathbf{x}, t)-\underbrace{\frac{\left[f_{j}(\mathbf{x}, t)-f_{j}^{e q}(\mathbf{x}, t)\right]}{\tau}}_{\text {Collision }}}_{\text {Streaming }} .
$$

The collision operator is simplified in the Bhatnagar-Gross-Krook (BGK) model by using a single relaxation parameter $\tau(t s)$ for all directions. $\tau$ indicates the rate at which the 
system approaches equilibrium through collision. The kinematic viscosity $v$ of the fluid is linked with the relaxation parameter (Qian et. al. 1992):

$$
v=c_{s}^{2}\left(\tau-\frac{1}{2}\right) .
$$

Kinematic viscosity is one of the key fluid properties that controls the flow behavior. It is often desired to have low kinematic viscosity to achieve higher Re number (inertial flow regime) in flow simulations. Since the relaxation parameter $\tau$ can not be smaller than 0.5 , this limits the LBMs ability to achieve very high $R e$ flow regimes. In this dissertation, moderately high Re number in the range of 1000 is achieved to simulate inertial flow regime in porous media with conduits. This is thought to be quite high for ground water.

In ground water, flow is generally driven by body force (gravity) or hydraulic pressure boundary conditions. For the simulations of fluid flow, either periodic, constant flux, or pressure boundary conditions are applied. For the pressure and flux boundaries, the methods of Zou and He (1997) are adopted, in which incoming $f$ s are computed based on the desired pressure or flux and the known $f$ s. The body force is applied by altering the macroscopic flow velocity, which is subsequently used to calculate $f^{q q}$.

\subsection{Passive scalar LBM}

Flekkøy (1993) introduced a LBM for isothermal, binary, miscible fluid mixtures, which can be used for the simulation of fluid flows with heat or mass transport. In this approach, two components, $\mathrm{A}$ and $\mathrm{B}$, are assumed and one is a very small fraction of the other. Therefore, collision between A-B or B-A is assumed negligible and not included in 
the computation. Component A will have the same equilibrium function as shown in equation (7) (i.e., it will behave as a regular fluid), but component B will evolve towards a new equilibrium as expressed by (Inamuro, 2002)

$$
f_{B j}^{e q}=t_{j}^{*} \rho_{B}(\mathbf{x})\left(c_{s}^{2}+\mathbf{e}_{j} \mathbf{u}_{A}\right) .
$$

The equilibrium distribution function represented by (10) recovers the advectiondiffusion governing equation for mass transport in moving fluids. The density (concentration) $\rho_{\mathrm{B}}$ for component B is computed following equation (5), and its velocity $\mathbf{u}_{\mathbf{A}}$ is assigned from component A; B is advected as a passive scalar. Hence the solute component $\mathrm{B}$ is called a passive component.

Component B has mass diffusivity $D_{m}$ expressed in terms of relaxation time $\tau_{\mathrm{B}}$ :

$$
D_{m}=c_{s}^{2}\left(\tau_{B}-\frac{1}{2}\right)
$$

Stockman et. al. (1998) demonstrated the ability of this LB model to simulate Taylor dispersion (mixing of solute due to molecular diffusion and non-uniform velocity field). Yoshino and Inamuro (2003) showed the ability of this model to simulate flow and breakthrough curves in a three-dimensional porous medium at different Reynolds numbers, and proposed boundary conditions for solute transport.

In this dissertation, three types of boundary conditions are implemented for solute components; the first is constant concentration and the second is zero-concentrationgradient, which allows advective flux but prohibits diffusive and dispersive fluxes. Much 
like the Zou and He (1997) boundaries applied to the flow, the constant concentration boundary is based on ensuring that the sum of the unknown, incoming $f \mathrm{~s}$, plus the known $f \mathrm{~s}$, equal the desired concentration (Inamuro et. al., 2002). The zero-concentrationgradient boundary requires that the solute $f \mathrm{~s}$ on each side of the boundary node are balanced; Sukop and Thorne (2006) contains more detailed descriptions of these boundaries. A mixed or third-type boundary condition for solute transport is also formulated for the LBM and explained in the following chapter. The ability of the passive scalar transport model to link directly with the BGK flow model of section 3.1 makes the simulation of transport in complex inertial flows eddies possible and represents a significant advance of flow and transport in karst aquifers.

\subsection{Macroscopic flow model in the LBM}

Flow and transport in porous media can be studied at pore scale, laboratory scale, and field scale. With change in scale, dominant processes and macroscopic governing equations may also change (Kang et al, 2002). The standard BGK LB model solves the flow field at the scale of explicit open space and solids, be it caves, conduits, fractures, macropores, or regular pores. Simulation at pore scale becomes too computationally intensive when an entire aquifer is under study. Thus an alternative technique is desirable that simulates flow at any scale, as Darcy's law does, and retains the capability of the LBM to solve the Navier-Stokes in conduits.

A common method employed to solve such problems is to use macroscopic properties, such as permeability and hydraulic conductivity, which consider the overall 
effect of the porous medium on the flow. Solid particles in a porous medium offer resistance to flow and interconnectivity of the pores facilitate the flow through the porous medium. Thus Darcy's Law does not depend directly on the scale of the problem and a Darcy-based LBM avoids the scale problem. The nodes in the porous media model represent a volume of a porous medium that should be larger than a representative elementary volume (REV). Each node represents a volume of porous medium which contains numerous pore and solids (Freed, 1998). There are multiple ways to solve the Darcy flux at macroscopic scale using the LBM (Gao and Sharma, 1994; Spaid and Phelan, 1997; Dardis and McCloskey, 1998 a, b; Freed, 1998; Kang et al, 2002; Walsh and Saar, 2008). The most common approach is to introduce an external force by dynamically changing the local velocity during the collision step (Gao and Sharma, 1994; Spaid and Phelan, 1997; Freed, 1998).

Spaid and Phelan (1997) introduced an LB method to model fluid flow through porous media with open channels. This method includes an external dissipative forcing term in the macroscopic velocity calculation to compute the flow at porous nodes and a regular LB model is used to recover the Navier-Stokes equation in the conduit or open regions. This method can not simulate zero permeability (a node with infinite momentum sink) and there are stability issues near the interface of a porous and open region.

Martys (2001) introduced a linear body force in the continuous Boltzmann equation to incorporate the dissipative force due to the porous medium. Error analysis proved that this method is more stable than the method proposed by Spaid and Phelan 
(1997). Kang et. al. (2002) introduced a unified LB method to model unidirectional, steady flow through heterogeneous porous media. In this method, all nodes are porous media nodes with a permeability value. Solid walls have zero permeability and no-slip conditions are applied, whereas open regions have infinite permeability, and porous medium nodes have non-zero permeability. Fluid properties are volume-averaged and satisfy the mass and momentum equations. Based on this model, the effect of matrix can be neglected when the ratio between the porous matrix permeability and a fracture permeability calculated by the cubic law is less than $10^{-4}$.

\subsubsection{Partial bounce-back method}

In the LBM, the bounce-back approach is used to enforce no-slip conditions near solid nodes. This means that when a group of particles (represented by the particle distribution function, $f_{j}$ ) streams into a solid node, it bounces back in the opposite direction without any loss of momentum, which ensures a no-slip boundary. Dardis and McCloskey (1998 a, b) introduced a partial bounce-back scheme to simulate permeability in a porous medium. There is a damping factor $\left(n_{s}\right)$ at nodes in the LB equation that retards the evolution of the particle distribution function in the medium. This is a Partial bounce-back approach for simulation of porous media at macroscopic scale. This novel model can simulate at large scale without excessive computational effort: As in standard ground water models, Darcy's law is applied and simulation of flow inside individual pores is not attempted.

To implement the Dardis and McCloskey (1998 a, b) LBM for macroscopic porous media, there is an additional collision step after streaming and BGK 
collision $\Delta f_{j}^{B G K}\left(x-e_{j} \Delta t, t\right)$, which is denoted by $\Delta f_{j}^{P M}(x, t)$. The overall collide and stream algorithm can be written as

$$
f_{j}^{* *}(\mathbf{x}, t+\Delta t)=f_{j}^{*}\left(\mathbf{x}-\mathbf{e}_{j} \Delta t, t\right)+\Delta f_{j}^{B G K}\left(x-\mathbf{e}_{j} \Delta t, t\right)+\Delta f_{j}^{P M}(x, t)
$$

for $0 \leq j \leq 8$.

where

$$
\Delta f_{j}^{P M}(\mathbf{x}, t)=n_{s}(\mathbf{x})\left[f_{j+2}(\mathbf{x}, t)-f_{j}\left(\mathbf{x}-\mathbf{e}_{j} \Delta t, t\right)\right] .
$$

$n_{s}(\mathbf{x})$ is a parameter called the solid scatter density per lattice node $\mathbf{x}$, where $n_{s}(\mathbf{x}) \in[0,1]$. This additional collision term accounts for the effect of the porous medium on the fluid and redistributes the particle momentum. The normal fluid flow model can be recovered from this model for $n_{s}=0$, and for $n_{s}=1$, a no-slip (bounce back) boundary condition is achieved that effectively makes the medium impermeable. For values of $n_{s}$ between 0 and 1 , bounce back is partial, which makes the medium effectively permeable. This model can have a different $n_{s}$ value at each node in the domain.

\subsubsection{Altered-velocity method}

Darcy's law is used to compute the volumetric flux or Darcian velocity $q$ of fluid flowing under a constant head, $h$ or pressure gradient, $\nabla p$ across a porous medium of permeability $\mathbf{k}$ :

$$
\mathbf{q}=-\frac{\mathbf{k}}{v \rho} \nabla p
$$

where $\rho$ is the fluid density and $v$ is the kinematic viscosity of the fluid. Darcy's law is applied on the representative elementary volume (REV) scale and above, and gives an averaged macroscopic flux for a porous medium. A resistance force $\mathbf{R}$ can be related to the pressure drop across a porous medium and it could be a tensor to account for 
direction-dependent properties. Equation (14) can be written in terms of a resistance field R (Freed, 1998):

$$
\nabla p=-\rho \mathbf{R q}
$$

This model treats the porous medium as a resistance field and calculates the volumetric flux for a given pressure gradient, $\nabla p$. Freed (1998) defined this approach as an averaging of steady state Navier-Stokes equations over the local sub-volume in the Stokes regime, where viscous forces are replaced by the resistive force as shown in the RHS of equation (15).

An LB-based porous media model is an extension of the basic LBM that is obtained by altering the local macroscopic velocity during the collision step. Some new notation is introduced to define the change in macroscopic velocity: $\tilde{\mathbf{u}}$ is the pre-collision velocity, $\mathbf{u}^{\prime}$ is the post collision velocity and $\overline{\mathbf{u}}$ is the mean centered velocity. An external force $\mathbf{F}$ is

$$
\mathbf{F}=\frac{\rho}{\tau}\left(\mathbf{u}^{\prime}-\tilde{\mathbf{u}}\right)
$$

which is equivalent to the term $\rho \mathbf{R} \cdot \mathbf{u}$, and

$$
\frac{\rho}{\tau}\left(\mathbf{u}^{\prime}-\tilde{\mathbf{u}}\right)=\rho \mathbf{R} \cdot \mathbf{u}
$$

The resistance field is related to the permeability as

$$
\mathbf{R}=\boldsymbol{u k} \mathbf{k}^{-1}
$$

Mean centered velocity $(\overline{\mathbf{u}})$ should be used as the correct macroscopic velocity, and is calculated as 


$$
\overline{\mathbf{u}}=\left(1-\frac{1}{2 \tau}\right) \tilde{\mathbf{u}}+\frac{1}{2 \tau} \mathbf{u}^{\prime}
$$

where

$$
\mathbf{u}^{\prime}=\mathbf{G} \cdot \widetilde{\mathbf{u}}
$$

and (Freed, 1998)

$$
\mathbf{G}=\frac{1-\left(\tau-\frac{1}{2}\right) \mathbf{R}}{1+\frac{1}{2} \mathbf{R}}
$$

The algorithm for the porous media model is (Freed, 1998)

$$
\begin{aligned}
& f_{i}\left(\mathbf{x}+\mathbf{e}_{i} \Delta t, t+\Delta t\right)=f_{i}(\mathbf{x}, t)-\frac{f_{i}(\mathbf{x}, t)-f_{i}^{*}(\rho, \mathbf{u}, T)}{\tau} \\
& f_{i}^{*}(\rho, \mathbf{u}, T)=t_{j}^{*} \rho(\mathbf{x})\left[c_{s}^{2}+\mathbf{e}_{i} \cdot \mathbf{u}+\frac{3}{2}\left(\mathbf{e}_{i} \cdot \overline{\mathbf{u}}\right)^{2}-\frac{1}{2} \overline{\mathbf{u}}^{2}\right],
\end{aligned}
$$

where $\mathbf{u}^{\prime}$ and $\overline{\mathbf{u}}$ are given by (19) and (20). The resulting macroscopic hydrodynamics will be governed by

$$
\frac{\partial \rho}{\partial t}+\nabla \cdot(\rho \mathbf{u})=0
$$

and momentum equations at the Navier-Stokes scale

$$
\frac{\partial(\rho \mathbf{u})}{\partial t}+\nabla \cdot(\rho \mathbf{u u})=-\nabla p-\rho \mathbf{R} \cdot \mathbf{u}+\nabla \cdot[\rho v(\nabla \mathbf{u}+\mathbf{u} \nabla)]
$$

where the centered mean velocity, $\overline{\mathbf{u}}$, is the macroscopic Darcy flux (q). Equation (25) recovers Brinkman's equation when the inertial term is negligible (Freed, 1998). The LHS of equation (25) represents the total acceleration of moving fluid: time-dependent acceleration and space-dependent convective acceleration. These accelerations become 
zero for creeping flow as there is no time-dependent change in flow and there is no spacedependent change in the flow field. The last term on the RHS accounts for the Brinkman correction and it becomes negligible for creeping flow in porous media (Kang et. al., 2002). The only remaining terms are the first two terms on the RHS, which are the same as equation (15). Equation (15) can easily be written as equation (14) using equation (18), hence the modified Navier-Stokes equation as shown in equation (25) is a form of Darcy's Law in laminar regime. The regular LB model (Eqs (7) and (8)) solves the Navier-Stokes equation. Hence the $f_{j}^{e q}(\mathbf{x})$ is modified to include a resistance term so that the LBM solves the modified Navier-Stokes equation represented by equation (25). Equation (25) solves the modified Navier-Stokes equation or macroscopic flow field, which is also given by equation (14), depending upon the value of $\mathbf{R}$. If the $\mathbf{R}$ value is zero, the node does not cause any resistance to flow and the equation (25) solves the Navier-Stokes equation; when $\mathbf{R}$ has a non-zero value, equation (25) solves the Darcy equation with permeability at a node given by equation (18). Thus, other than the value of $\mathbf{R}$, no explicit changes necessary to switch between the Navier-Stokes equation and Darcy equations in different regions of the simulation domain.

\subsubsection{Validation of flow model}

Permeability is a characteristic property of any porous medium. Permeability controls the flow rate for any head gradient across the domain, assuming the viscosity and density of fluid are constant. Prediction of permeability is an important aspect of hydro-geological modeling for the correct estimation of reservoir yield. 


\subsubsection{Permeability test}

Flow was simulated through a porous medium $(100 l u \times 5 l u)$, with kinematic viscosity of fluid $0.0333 \mathrm{lu}^{2} / \mathrm{ts}$ and uniform resistance field $\mathbf{R}$ set equal to $0.1 \mathrm{ts}^{-1}$. The permeability predicted using equation (18) is $0.3333 l u^{2}$. The left and right boundaries were maintained at a pressure of $0.333366 m u-l u^{-1} t^{-2}$ and $0.33333 m u-l u^{-1} t^{-2}$. The average flux $(\rho u)$ was computed over the whole domain and used in Darcy's law as shown in equation (14) to compute the permeability $(k)$, which was found to be $0.3333 l u^{2}$. After scaling by physical size the model is ready to solve real ground water problems (as shown in Alvarez (2007), Anwar and Sukop (2008), Variano et. al. 2008 (to be submitted)).

A simple test to validate this model is to examine the flux across a porous medium for a certain pressure drop. Darcy's law is valid only for the laminar regime of flow at low $R e$. For higher $R e$, the inertial term becomes important for flow, and flux can not be accurately predicted by Darcy's law.

\subsubsection{Permeability test at varying hydraulic gradients}

Test simulations are conducted on a $100 l u \times 5 l u$ domain for uniform $\mathbf{R}=0.1 t^{-1}$

and $\tau=1$ ts. Pressure boundary conditions (Zou and He, 1992) are applied at the left and right boundaries and the domain is periodic in the transverse direction. 


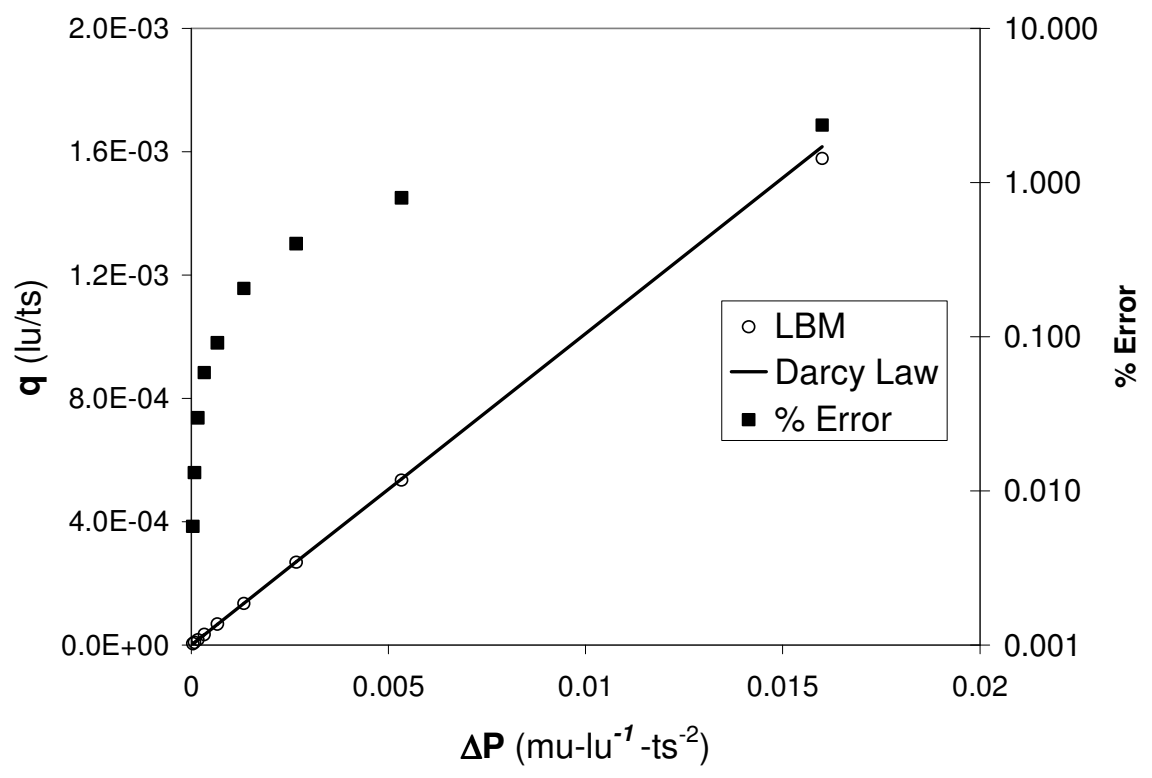

Figure 9. Flux is plotted against the pressure difference across the domain. Deviation from Darcy's Law grows significantly as pressure difference and flux increase.

This is a common test to validate the effect of resistance on the flow exerted by porous media. In this case, resistance $\mathbf{R}$ has a non-zero value at every node. Flux is plotted against pressure difference across the domain in Figure 9. As shown in Figure 9, flux linearly increases with increase in pressure gradient but starts to deviate from the value predicted by Darcy's law. This is due to the emergence of inertial effects at higher flow rate, which are not considered by Darcy's Law. Extension of Darcy's law, for example the Darcy-Forchheimer equation, account for the inertial effects and the LBM macroscopic flow models are also capable of solving for the inertial effects. See Alvarez (2007) for similar results based on explicit pore-solid Navier-Stokes modeling. 


\subsubsection{Zero and infinite resistance test}

There is another test for flow between parallel plates for which an analytical solution is available. In this case $\mathbf{R}$ has zero value over the whole domain except at the boundaries in the direction transverse to the flow direction. The analytical solution for flow under a pressure gradient between parallel plates is expressed as (Kang et. al. 2002)

$$
u(y)=\frac{a^{2} \Delta p}{2 \mu l}\left[\frac{y}{a}-\left(\frac{y}{a}\right)^{2}\right]
$$

where $a$ is the width between the parallel plates, $y$ is position in the channel, which varies between 0 and $a$, and $\mu$ is dynamic viscosity of fluid. $\Delta \mathrm{p}$ is the pressure difference across the domain of length $l$. The test simulation is conducted on a $50 l u \times 20 l u$ domain under a pressure difference of $0.003 m u-l u^{-1}-t s^{-2}$ and $\tau=1 t s$.

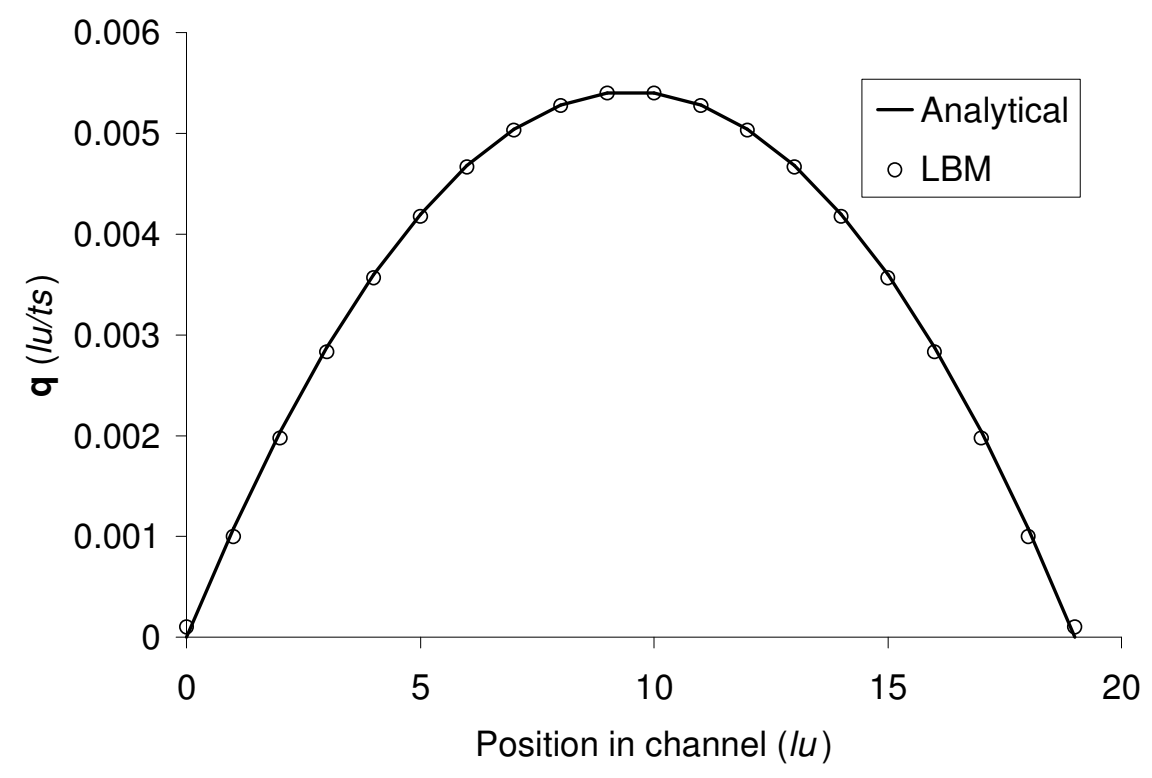

Figure 10. Poiseuille flow profile between the parallel plates using altered-velocity flow model. 
The velocity profile obtained from the LBM and compared with the analytical solution expressed by equation (26) is shown in Figure 10. The two solutions match very well and validate the ability of the LBM-based Darcy's law solver to simulate no-slip conditions on a solid wall where $\mathbf{R} \rightarrow \infty$ and Poiseuille flow (Navier-Stokes equation) at nodes where $\mathbf{R}=0$.

\subsubsection{Filled parallel plate test}

There is another interesting example similar to Figure 10 in which the space between parallel plates is filled with homogeneous porous medium with known permeability. A domain of $50 l u \times 20 l u$ is also used for this test simulation and the fluid has a kinematic viscosity of $0.166 \mathrm{lu}^{2} / \mathrm{ts}$. Pressure boundaries are maintained on the left and right boundaries with a pressure difference of $0.001 \mathrm{mu} / \mathrm{lu} \mathrm{u}^{1}-\mathrm{ts} \mathrm{s}^{2}$. Unlike Figure 10, flow becomes uniform in the porous medium and falls to zero at the wall. Figure 11 represents the flow between parallel plates filled with porous media of $\mathbf{R}=1 \mathrm{ts}^{-1}$. 


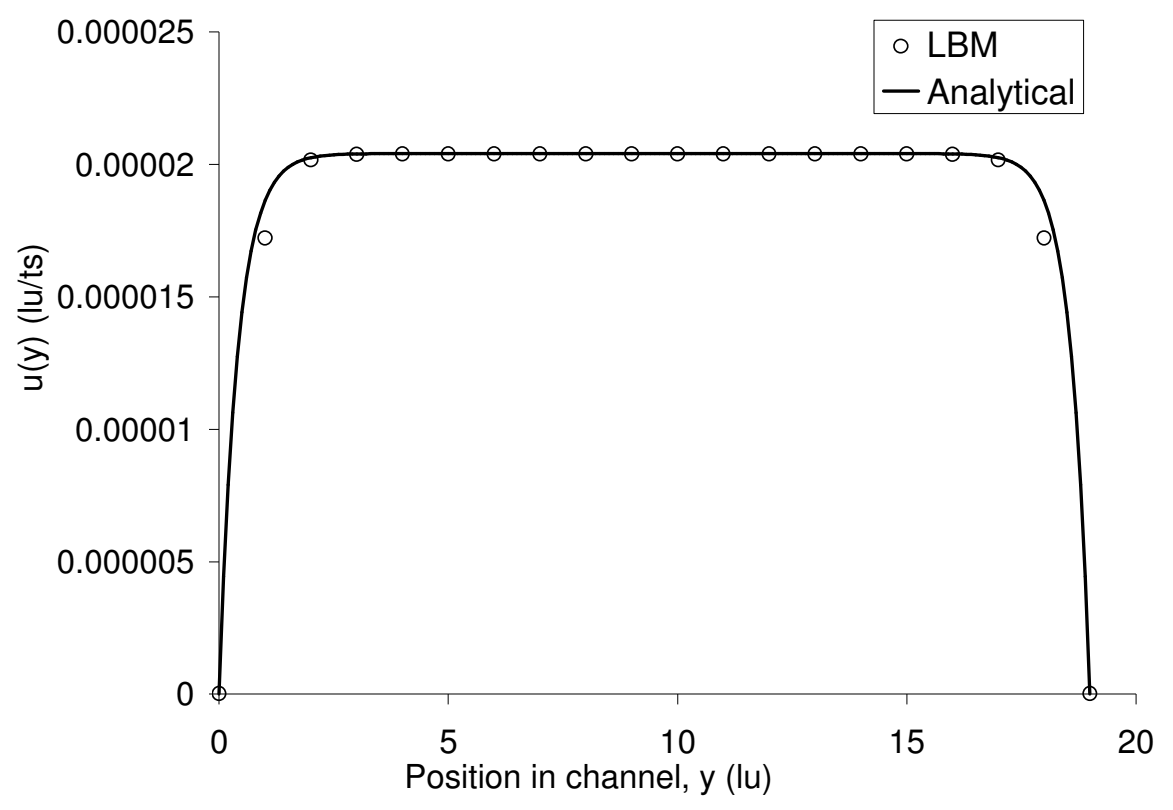

Figure 11. Flow profile between parallel plates filled with porous media of $R=1 t^{-1}$.

As shown in Figure 11, there is a uniform flow across the width of the channel depending upon the permeability of the region and velocity drops to zero (no-slip) near the solid nodes assigned at two boundaries along the flow direction.

\subsection{Anisotropic dispersion solver in the LBM}

The traditional governing equation for mass transport of a solute subjected to advection and anisotropic dispersion in porous media is a partial differential equation called the Anisotropic Advection-Dispersion Equation (AADE).

$$
\frac{\partial C}{\partial t}+\nabla \cdot \mathbf{u} C=\nabla \bullet \mathbf{D} \nabla C
$$


where $C$ is solute concentration $\left(M L^{-3}\right), \mathbf{u}=\mathbf{q} / n$ is mean pore velocity $\left(L T^{-1}\right), \mathbf{q}$ is Darcy flux $\left(L T^{l}\right), n$ is porosity, $\mathbf{D}_{i j}$ is the dispersion coefficient $\left(L^{2} T^{-1}\right)$ in the $i j$ direction, $x$ and $y$ are spatial coordinates $(L)$, and $t$ is time $(T)$.

Advection and mechanical dispersion are typically the dominant mechanisms for transport of solute in granular media. The process of mechanical dispersion is anisotropic even if the porous medium is isotropic with respect to grain size and hydraulic conductivity, because flow in the longitudinal direction is dominant and that stretches the solute plume into an elliptical shape (Freeze and Cherry, 1979). The passive scalar LBM for solute transport introduced earlier uses the simplest form of collision mechanism and has a single, lumped relaxation time for all directions that gives isotropic diffusion. This is appropriate for the simulation of diffusion in free flowing fluids. However dispersion in porous media is inherently anisotropic and an anisotropic dispersion solver is needed to develop a LBM with capabilities comparable to those of standard porous media solute transport solvers.

Zhang et al. (2002 a, b) and Ginzburg (2005) introduced LBMs with directiondependent relaxation parameters for the solute component in order to simulate anisotropic dispersion in porous media. Here, we apply the model proposed by Zhang et al (2002 a), which has four relaxation parameters in nine directions. Conservation of mass is ensured by taking a weighted summation of the particle distribution function, $f_{j}$ so that the collision step remains mass invariant (Zhang et al, 2002 a): 


$$
\rho_{B}=\sum_{j} \frac{f_{B j}}{\tau_{B j}}\left(\sum_{j} \frac{w_{j}}{\tau_{B j}}\right)^{-1}=\sum_{j} f_{B j}^{e q},
$$

where $w_{j}$ is the weighting factor in the $j$ direction and the dispersion tensor in terms of relaxation parameters $\left(\tau_{B j}\right)$ is (Zhang et al, $\left.2002 \mathrm{a}\right)$

$$
\begin{aligned}
& D_{x x}=\frac{1}{18}\left[4 \tau_{1}+\tau_{5}+\tau_{6}-3\right] \\
& D_{y y}=\frac{1}{18}\left[4 \tau_{2}+\tau_{5}+\tau_{6}-3\right] . \\
& D_{x y}=D_{y x}=\frac{1}{18}\left[\tau_{5}-\tau_{6}\right]
\end{aligned}
$$

The dispersion coefficients in equation (29) are found using the following equation (Bear 1972)

$$
D_{i j}=\alpha_{T} \sqrt{u_{x}^{2}+u_{y}^{2}} \delta_{i j}+\frac{\left(\alpha_{L}-\alpha_{T}\right) u_{i} u_{j}}{\sqrt{u_{x}^{2}+u_{y}^{2}}}
$$

where $\delta_{i j}$ is Kronecker delta, and $\alpha_{L}$ and $\alpha_{T}$ are dispersivities in the longitudinal and transverse directions respectively. $i$ and $j$ represent the Cartesian directions (x or y). Dispersivity is a medium property and changes with time and space; however dispersivity reaches a asymptotic value after some distance/time. Equation (30) is an empirical relationship to approximately quantify the dispersion tensor (Hassanizadeh, 1996). For heterogeneous domains, velocity $\left(u_{x}, u_{y}\right)$ changes at every node, so the dispersion coefficient does too.

The collision step is changed from that for a diffusion model to one that imposes a macroscopic anisotropic dispersion model, only for the solute component in the porous matrix. This will provide a LBM-based anisotropic dispersion solver comparable to those 
found in standard ground water model, and LB model also retains the potential to solve the Navier-Stokes and advection-diffusion equations in conduits.

\subsubsection{One-dimensional solute transport with anisotropic dispersion model}

This model is verified against the analytical solutions given in StAnMod

(Simunek et. al. 1999) in one and two-dimensions. A one-dimensional numerical experiment is set up in a $400 l u \times 4 l u$ domain with constant flux boundary $(0.01 l u / t s)$ at each end. As an initial condition, the domain had a slug of solute over the whole width of the domain at $10 l u$ from the flow boundary. Dispersivity equal to $1 l u$ is set at every node. The spatial breakthrough curve is measured at 10,000 ts and 30,000 ts and shown below in Figure 12. The analytical and LBM results show a good match for spatial breakthrough.

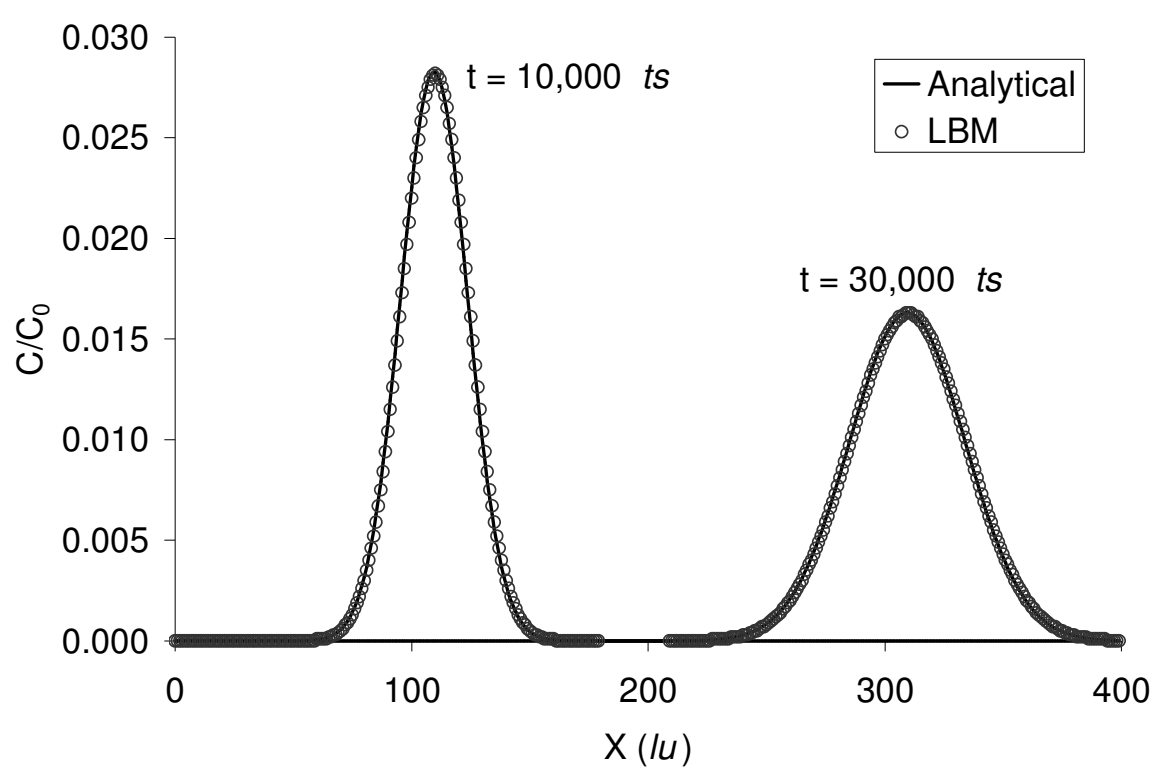

Figure 12. Spatial breakthrough curve for solute transport in one-dimensional flow field with uniform dispersivity $1 l u$. 


\subsubsection{Two-dimensional solute transport with anisotropic dispersion}

For uniform flow aligned with the x-axis in 2-dimensions, the anisotropic advection-dispersion equation can be written as (Bear, 1972)

$$
\frac{\partial C}{\partial t}+u \frac{\partial C}{\partial x}=\frac{\partial}{\partial x}\left(D_{x x} \frac{\partial C}{\partial x}\right)+\frac{\partial}{\partial y}\left(D_{y y} \frac{\partial C}{\partial y}\right)
$$

Another verification is established for a domain $(200 l u \times 20 l u)$ with anisotropic dispersivity. The one-dimensional flow field is set using constant flux $(0.01 \mathrm{lu} / \mathrm{ts})$ boundaries at the two ends. The dispersivity in the longitudinal $\left(D_{L}\right)$ and transverse $\left(D_{T}\right)$ directions at every node is set equal to $1 l u$ and $0.1 l u$ respectively. As an initial condition, the domain had a point solute concentration at coordinate $(10,0)$.

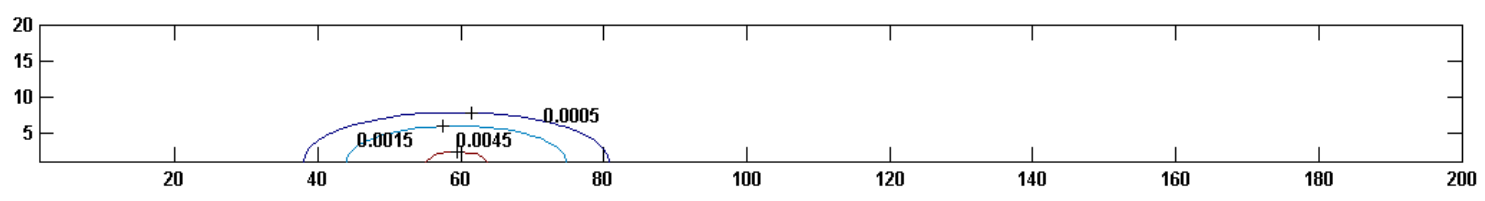

Figure 13. Solute plume for $u=0.01 l u / t s$ and $\mathrm{D}_{\mathrm{L}}=1 l u \mathrm{D}_{\mathrm{T}}=0.1 \mathrm{l} u$ in a $200 l u \times 20 l u$ domain after 5000 $t s$. Domain is periodic in vertical direction. Solute was introduced at $(10,0)$ at time $=0$.

The spatial breakthrough curve is measured at 5000 ts and compared with the analytical solution. The results shown below in Figure 14 demonstrate a good match between the analytical and LBM solutions. 


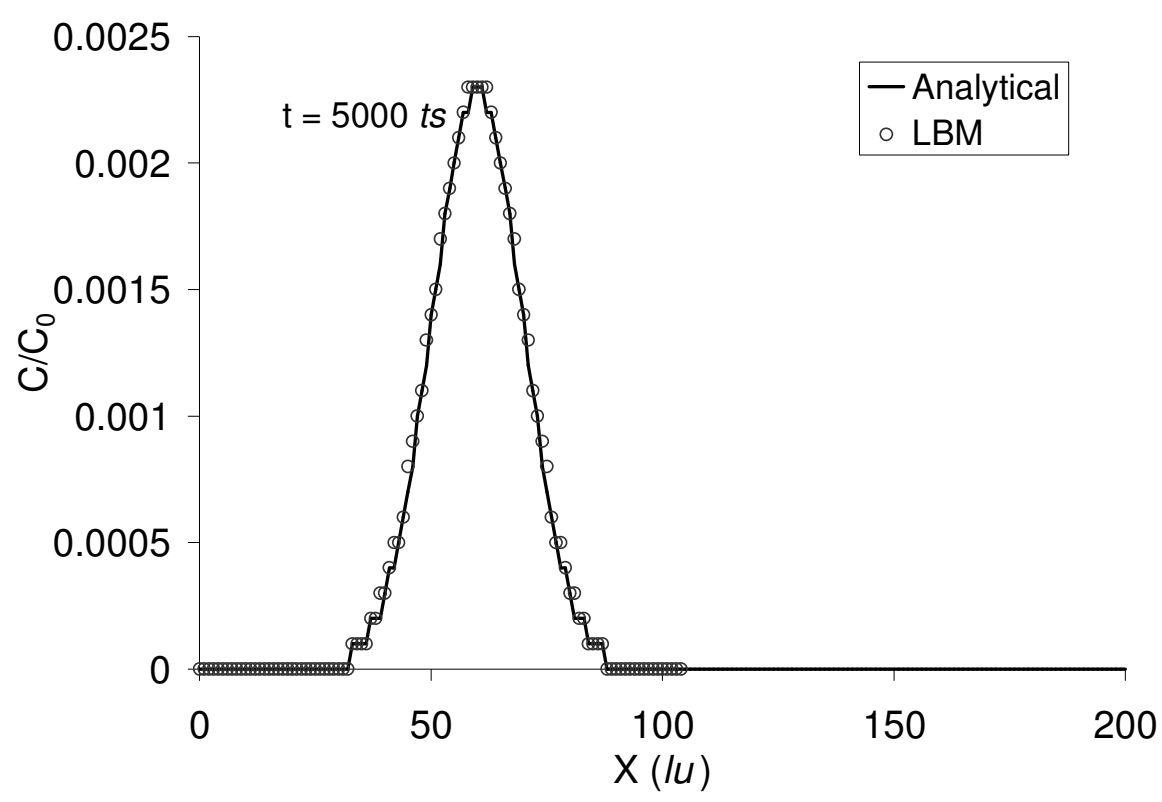

Figure 14. Spatial breakthrough curve for $D_{L}=1 l u$ and $D_{T}=0.1 l u$ in a $200 l u \times 20 l u$ domain.

\subsubsection{Two-dimensional solute transport with anisotropic dispersion for non-axis- aligned flow field}

the LBM for solute transport with anisotropic dispersion is tested in a $200 l u \times$ $100 l u$ domain with velocity boundary conditions in the longitudinal direction and periodic boundaries in the transverse direction. At every node, the fluid component has velocity in the $x\left(u_{x}=0.01 \mathrm{lu} / \mathrm{ts}\right)$ and $y\left(u_{y}=0.005 \mathrm{lu} / \mathrm{ts}\right)$ direction, which causes the resultant flow to be in a diagonal direction. The resultant velocity is $\sim 0.0112 \mathrm{lu} / \mathrm{ts}$ along an axis inclined at $\sim 27^{\circ}\left(\tan ^{-1}(1 / 2)\right)$ from the $x$-axis. This velocity is used as the rate of advective solute transport in the analytical solution. The longitudinal dispersivity is set equal to $0.5 l u$ and transverse dispersivity equal to $0.1 l u$ at every node. The dispersion tensor is calculated using equation (30). The solute is introduced at node $(10,10)$. The contour map of solute concentration at 15,000 ts is shown in Figure 15 and shows good agreement with the simulated analytical solution. 


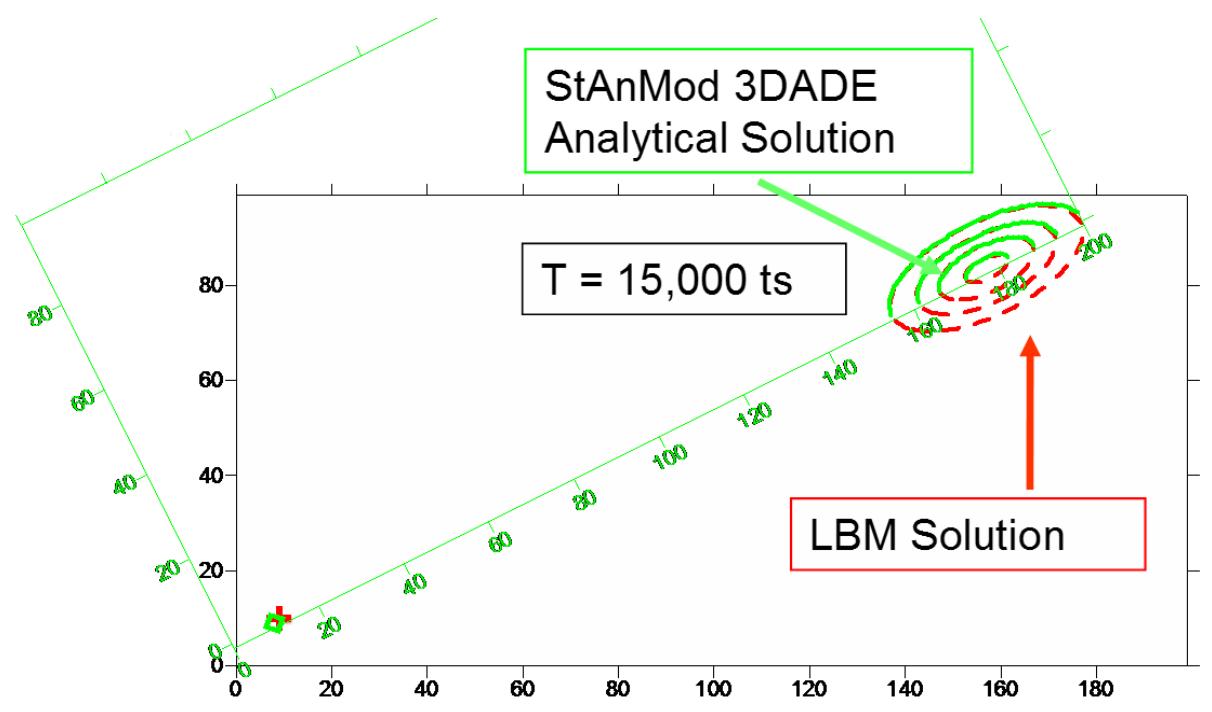

Figure 15. Contour map of solute concentration in flow field unaligned with the LBM coordinates $\left(u_{x}\right.$ $=0.01 l u / t s$ and $\left.u_{y}=0.005 l u / t s\right)$ with $D_{L}=0.5 l u$ and $D_{T}=0.1 l u$. The LBM solution is shown in red and analytical solution is shown in green for comparison.

This verifies the ability of the LBM to simulate anisotropic solute dispersion in a flow field that is not aligned with the coordinate axes of the LBM and suggests that the model will work in more complex flow fields.

\subsection{LBM on non-uniform grids}

The Boltzmann equation can be written in differential form as follows:

$$
\frac{\partial f}{\partial t}+\mathbf{e} \nabla f=\Omega
$$

where $f$ is particle distribution function, $\mathbf{e}$ is the microscopic velocity of particles, and $\Omega$ represents the collision term (Wolf-Gladrow, 2000). Equation (8) is a finite difference form of equation (32) where the collision term is the simplified Bhatnagar-Gross-Krook (BGK)-type with a single rate of relaxation. 
Since the particle distribution $f$ in equation (32)(33) is a continuous function in both space and time, the LBM can be applied on non-uniform grids. There are several models available in the literature for non-uniform meshing in the LBM. The first effort to develop lattice Boltzmann models for non-uniform grids was the merging of the lattice Boltzmann equation with finite difference or finite volume methods (Succi, 2001). The particle distribution function was interpolated to the non-uniform grid using different orders of interpolation such as piecewise-constant, linear, or parabolic interpolation over space/time. He et al. (1996) proposed an interpolation-supplemented LBM, where the particle distribution functions are interpolated to the non-uniform grids at every time step. The differential lattice Boltzmann equation is solved on the non-uniform grids using coordinate transformation.

Chen (1998) has shown that finite-volume-based LBM can recover conservation laws with high accuracy. Chen (1998) solved the discrete lattice Boltzmann equation (8) using an upwind scheme, the accuracy of the result depends on the selected numerical method. Fillipova and Hänel (1998) applied the concept of hierarchical grid refinement as widely used in conventional computational fluid dynamics methods. The computational domain is discretized into a coarse grid and a finer grid is superimposed onto the coarse grid by adaptation criteria or defined a priori. The time step is also non-uniform over the coarse and fine grids. Shu et al. (2001) used Taylor-series and the Runga-Kutta method to solve for the particle distribution function over the non-uniform grid using the known particle distribution function at the square grid. All these models have been tested for benchmark problems such as Poiseuille flow, lid-driven cavity flow, flow around a 
cylinder, etc. The LB models used in this dissertation could be computed on non-uniform meshes in time and space. This could improve results in the complicated geometries found in karst aquifers.

\section{BOUNDARY VALUE PROBLEMS IN SOLUTE TRANSPORT}

This chapter presents more elaborate tests of the LBM solute transport algorithm and its boundary conditions against established analytical solutions for a variety of boundary conditions and domain types. Two different domain types are chosen for the tests: finite and semi-infinite. Two different types of solute boundary conditions (constant concentration and constant total solute mass flux) are applied at the inlet boundary for these two domain types and zero concentration gradient conditions are maintained at the exit boundaries. These conditions correspond to four different established analytical solutions (Table 1) that differ significantly at low values of a non-dimensional number called the Peclet number. The simulated concentration breakthrough curves are compared against the analytical solutions. The results of these tests further verifies the LBM's solute transport capabilities and opens a path for its application to more complex problems as found in karstic aquifers.

\subsection{Methodology}

Lattice Boltzmann models can be thought of as having their own consistent set of dimensional units in terms of lattice units $(l u)$ and lattice time steps $(t s)$, which correspond to space $(\mathbf{x})$ and time $(t)$ respectively. To compare any physical system with any numerical simulation (e.g., LBM) it is convenient to relate them by non-dimensional numbers like the Reynolds number (for fluid flow), the Peclet number (for transport 
problems), non-dimensional relative concentration (concentration relative to input concentration, $\mathrm{C} / \mathrm{C}_{0}$ ), and the number of pore volumes for scaling pulse duration. The Peclet number $\left(P_{e}\right)$ (as defined in section 2.5) is a measure of the balance between diffusion and advection rate during solute transport as shown in equation (2). This can be used to compare simulation results with laboratory or field data. The relative concentration is defined as (van Genuchten and Wierenga, 1986)

$$
C(x, t)=\frac{C(x, t)-C_{i}}{C_{0}-C_{i}},
$$

where $C_{0}$ is the concentration of the applied solution and $C_{\mathrm{i}}$ is the initial concentration, which is set here to zero, so $C(x, t)=C(x, t) / C_{0}$.

A pore volume, $T$, is the volume of water contained in a porous medium (van Genuchten and Wierenga, 1986). For a column with cross-sectional area $A$ and volumetric water content $\theta$, the number of pore volumes is calculated by dividing the volume of water flushed through the column by the liquid capacity $\left(V_{0}=A \theta L\right)$ of the column. $\mathrm{L}$ is the length of porous medium.

$$
T=\frac{V}{V_{0}}=\frac{A \theta u t}{A \theta L}=\frac{u t}{L} .
$$

Both relative concentration and pore volume are dimensionless and can be used to present non-dimensional breakthrough curves. Thus, physical and LBM breakthrough curves can be compared directly when they are presented this way.

\subsection{Zero-diffusion transport}

Transport of inert solute is generally governed by advection and diffusion. Zerodiffusion transport means solute is transported by movement of the solvent only and does 
not undergo any diffusion. The analytical solution for zero-diffusion breakthrough curves in gravity-driven Poiseuille flow in a slit is derived below. Though diffusion is never absent in solute transport, it can be infinitesimally small in fast flows such as those that can occur in karst aquifers. With its analytical solution, this zero-diffusion problem provides an excellent test of the LBM's ability to solve such high $P e$ problems. This kind of situation can appear in a system of fast moving carrier fluid where the mechanical and molecular spreading of solute are low relative to the flow.

Figure 16 shows the fully developed parabolic velocity profile for laminar flow in a slit. Because negligible diffusion is assumed, the solute front will have the same shape as the velocity profile. Using the equivalence between velocity profile and solute front, relative concentration $\left(\mathrm{C} / \mathrm{C}_{0}\right)$ at any cross-section through the slit can be computed and the analytical breakthrough curve as a function of time can be computed.

As shown in Figure 16, $a$ is half the width of the slit, and $\mathrm{P}_{2}(0, a)$ represents a point lying on the parabolic solute profile at the slit cross-section where solute is introduced in to a stable Poiseuille flow. $\mathrm{L}$ is the distance from the solute inlet where breakthrough is measured. $P_{1}(L, w)$ is the point lying on the parabolic solute/velocity profile at the cross-section where the breakthrough curve is measured, and $P_{0}\left(x_{\max }, 0\right)$ is the point lying on the vertex of the parabola. The relative concentration $C / C_{0}$ at $\mathbf{x}=\mathrm{L}$ will be determined by the half-width $w$ of the solute parabola relative to the half-width of the slit $a$. 


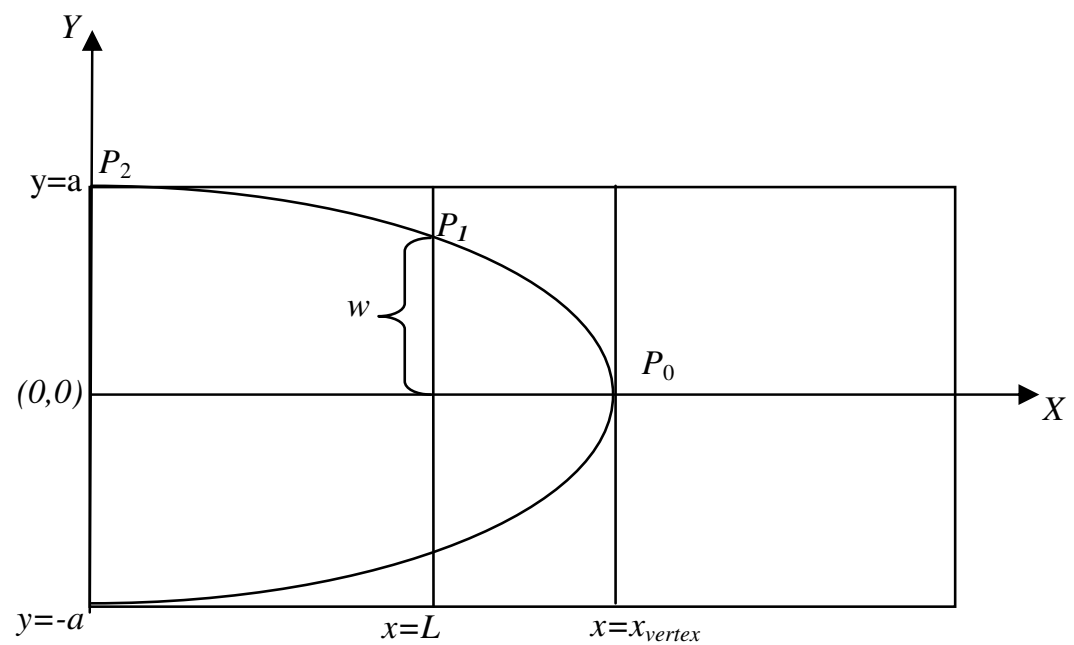

Figure 16. Parabolic velocity profile in laminar flow.

Equation (35) is a general equation for the parabola shown in Figure 16, where $k$ is some constant and $x_{\text {vertex }}$ is the distance of the vertex of the parabola from the origin at the inlet:

$$
\begin{aligned}
& x-x_{\text {vertex }}=k y^{2} \\
& \frac{x-x_{\text {vertex }}}{y^{2}}=k .
\end{aligned}
$$

The $x$ coordinate of the vertex at time $t$ is

$$
x_{\text {vertex }}=u_{0} t
$$

where $u_{0}$ is maximum velocity, which can be determined from the Poiseuille law for a gravity-driven flow as (Tritton, 1988)

$$
u_{0}=\frac{g a^{2}}{2 v},
$$

where $g$ is gravity and $v$ is kinematic viscosity. Inserting the points $\mathrm{P}_{2}(0, a)$ and $\mathrm{P}_{1}(L$, $w$ ) into equation (35) and solving for $w$ yields 


$$
\begin{gathered}
\frac{0-x_{\text {vertex }}}{a^{2}}=k=\frac{L-x_{\text {vertex }}}{w} \text { or } \\
w=a \sqrt{\left(\frac{x_{\text {vertex }}-L}{x_{\text {vertex }}}\right) .}
\end{gathered}
$$

Finally, the breakthrough curve for pure advection in a slit under Poiseuille flow is given by

$$
\frac{C}{C_{0}}=\frac{w}{a}=\sqrt{1-\frac{L}{x_{\text {vertex }}}}=\sqrt{1-\frac{2 L v}{g a^{2} t}} .
$$

For pipe flow, the concentration at any section will be equal to area of a circular disc having a radius equal to half the width of the parabolic section ( $w$ in Figure 16). Hence, the relative concentration for pipe flow at any cross-section is equal to $\frac{\pi w^{2}}{\pi a^{2}}$ and $\frac{C}{C_{0}}=\frac{\pi w^{2}}{\pi a^{2}}=1-\frac{L}{x_{\text {vertex }}}$ (Taylor, 1954). Again $x_{\text {vertex }}$ is the distance of the parabolic front from inlet at any time $t, x_{v e r t e x}=u_{0} t$ where now $u_{0}$ is (Tritton, 1988) $u_{0}=\frac{g a^{2}}{4 v}$. Finally, the breakthrough curve for pure advective flow in a pipe under gravity-driven Poiseuille flow is (Kutilek and Nielsen, 1994)

$$
\frac{C}{C_{0}}=1-\frac{4 L v}{g a^{2} t}
$$

\subsection{Diffusion and Taylor dispersion}

Diffusion is an inevitable physical phenomenon observed when solute is introduced into fluid. It is a spontaneous process induced by thermal motion of solute and fluid molecules. Owing to diffusion, solute moves from zones of higher concentration towards lower concentration, which tends to make the distribution of solute concentration 
uniform in a domain over time. To a first approximation as stated by Fick's law, the diffusive flux of solute is directly proportional to the concentration gradient of solute, and can be written as

$$
J_{D}=-D_{m} \frac{\partial C}{\partial x}
$$

where $D_{m}$ is the molecular diffusion coefficient, $C$ is the volume averaged concentration, and $x$ is the spatial coordinate.

Taylor (1954) introduced the concept of solute dispersion to explain the symmetrical spreading of solute in experiments where a salt is injected into a stream of solvent flowing through a tube. Dispersion is the spreading of solute due to the combined action of fluid velocity variations and molecular diffusion. Taylor explained that if advection is slow and fluid takes a long time to travel through the tube, then solute could diffuse appreciably across and along the direction of flow. If flow is fast and solvent travels through the pipe quickly, then solute will not have enough time to diffuse significantly. These end member conditions give bounds for the applicability of Taylor's dispersion equation. These bounds should be satisfied for the prediction of effective diffusion (i.e., dispersion) based on molecular diffusion, fluid velocity, and radius of a pipe or width of a slit.

Taylor Dispersion is the apparent mixing of solute dissolved in a fluid that flows through a slit or tube in accordance with Poiseuille's equation. It results from a combination of velocity variations and diffusion. When the Peclet number is high enough, dispersion under these conditions is mainly a result of velocity variations that 
occur due to no-slip wall boundaries, which lead to Poiseuille flow and a velocity gradient transverse to the direction of flow. Dispersion caused by such variations in velocity is called mechanical dispersion (Fried and Combarnous, 1971; Bear, 1972; Freeze and Cherry, 1979) and occurs at all scales; for example, it also occurs due to macroscopic medium heterogeneity in porous media such as meter plus scale layering of geological units. Dispersive transport is generally described using the assumption that it follows the same Fickian form as Equation (38):

$$
J_{h}=-D_{h} \frac{\partial C}{\partial x}
$$

$D_{h}$ is the mechanical dispersion coefficient (Bear, 1972) or the apparent diffusion coefficient (Aris, 1956). The basic idea is that in a shear (transversely varying) flow, a cloud of solute will be longitudinally stretched more in some regions of the flow field than in others. The resulting transverse irregularities of the cloud are then smoothed by diffusion in the transverse direction. The net result is that the cloud appears to be diffusing in the longitudinal direction at a rate that is much faster than what would be predicted by molecular diffusion alone. This effect does not occur in a uniform flow field in free-flowing fluid, though it does in macroscopically uniform flow in a porous medium as discussed previously. Taylor (1954) and Aris (1956) gave the following equation for the effective diffusion $D$ in a pipe:

$$
D=D_{m}+\frac{u^{2} a^{2}}{48 D_{m}} .
$$

This expression for effective diffusion remains valid for flow in a pipe when the following criteria are met (Taylor, 1954):

$$
\frac{4 L}{a}>>\frac{u a}{D_{m}}>>\sqrt{48} .
$$


Here $a$ is the radius of the pipe, $D_{m}$ is molecular diffusion, $u$ is average velocity, and $L$ is the length of tube over which appreciable changes of concentration occur. Similar bounds for effective diffusion for flow in a slit are available (Aris, 1956):

$$
\frac{4 L}{a}>>\frac{u a}{D_{m}}>>\sqrt{210 / 4}
$$

where $2 a$ is slit width.

These equations are important in that they allow a priori prediction of the dispersion coefficient for macroscopically 1-D transport in laminar flow in fracture and conduit systems and hence provide a unique case for verification of the LBM solute transport model under karst like conditions.

\subsection{Concentration injection and detection modes}

The concentration of solute can be expressed as resident (i.e., volume-averaged) concentration $C_{\mathrm{r}}\left(\mathrm{ML}^{-3}\right)$ in terms of the mass of solute per unit volume of pore fluid. In contrast, the flux concentration $C_{\mathrm{f}}\left(\frac{M}{L^{2} T} / \frac{L^{3}}{L^{2} T}=M L^{-3}\right)$ is defined as the flux of solute mass per volumetric flux of pore fluid exiting a plane normal to the direction of advective transport (Kreft and Zuber, 1978). There is a difference between these concentrations when there is a concentration gradient and hence a dispersive or diffusive flux. Such concentration gradients will be present at the advancing front and can also occur at the inflow boundary when the diffusion or dispersion is high relative to the velocity (i.e., low $P e$ ). These two types of concentration modes correspond to two different kinds of 
injection and detection modes. The transport governing equation remains unchanged for different injection modes, but $C$ is best viewed as $C_{\mathrm{r}}$ or $C_{\mathrm{f}}$ depending upon the type of injection and detection modes used.

The total mass flux at any cross-section is equal to mass flux due to advection and mass flux due to diffusion/dispersion:

$$
u C_{f}=u C_{r}-D \frac{d C_{r}}{d x}
$$

$u C_{r}$ represents advective flux and $-D \frac{\partial C_{r}}{\partial x}$ represents diffusive/dispersive flux. Solving equation (43) for $C_{f}$ gives the relationship between the flux and resident concentration

$$
C_{f}=C_{r}-\frac{D}{u} \frac{d C_{r}}{d x}
$$

Equation (44) is necessary only when $D \neq 0$ and $u \neq 0$. When $D=0$, which is the case of purely advective transport, or when $\frac{d C_{r}}{d x}=0$ (uniform concentration), $C_{f}=C_{\mathrm{r}}$. If $\mathrm{u}=$ 0 , there is pure diffusion.

The constant mass flux boundary or third-type boundary condition is implemented in the LBM code as follows. A concentration $C_{0}$ is prescribed for this boundary condition. The distribution functions at the boundary are computed the same way as for the constant concentration boundary condition (Sukop and Thorne, 2006) except that the concentration used to compute them is not $C_{0}$ but, instead, a $C_{r}$ computed from the condition 


$$
\left(-D \frac{\partial C_{r}}{\partial x}+u C_{r}\right)=u C_{0},
$$

which can be approximated in terms of concentrations at the boundary $(C)$ and first interior nodes $\left(C_{1}\right)$ as

$$
\left(-D \frac{C_{1}-C}{1}+u C\right)=u C_{0} \Rightarrow C=\frac{u C_{0}+D C_{1}}{D+u} .
$$

This is equivalent to specifying a constant flux of solute mass at the boundary and differs from simply specifying a constant concentration $C_{0}$ at the boundary as in the Drichlet or first-type boundary.

\subsection{Boundary conditions}

The governing equation for advection and diffusion/dispersion in one-dimension is:

$$
\frac{\partial C}{\partial t}+u \frac{\partial C}{\partial x}=D \frac{\partial^{2} C}{\partial x^{2}}
$$

$u$ is the average pore water velocity in the flow direction $x$. Equation (47) can be applied to finite or semi-infinite domains. In this study, two different types of inlet boundary conditions in finite and semi-infinite domains are considered: first-type (constant concentration) and third-type (constant flux boundary) inlet boundary conditions. A zero concentration-gradient boundary is used at the exit boundary.

There are two different methods by which solute could be introduced into a domain at its inlet. Either there is some constant concentration (first-type) at the inlet that does not undergo any dispersion or diffusion at $x=0$, but at $x=0+(\mathrm{x}>0)$ it undergoes all the physical processes like advection diffusion and dispersion, or the solute is 
introduced into the domain while undergoing advection as well as dispersion at the inlet. This is a constant flux boundary (third-type) similar to Cauchy's mixed boundary condition.

The LBM can simulate constant concentration and constant flux boundary conditions for semi-infinite and finite domains. First-type boundary conditions are simple constant concentration boundaries. Third-type boundary conditions represent a situation where constant mass flux is maintained. These include an advective flux and a diffusive/dispersive flux when there is a concentration gradient at the inlet. This boundary often represents real situations more closely than a first type boundary condition because it is natural to expect some diffusion due to concentration gradients at the inlet section when mass is first introduced. It is usually the chemical flux rather than the concentration that is held constant when a solution of a particular concentration is input into a domain at a constant rate. The boundary condition can be stated as

$$
\left(-D \frac{\partial C_{r}}{\partial x}+u C_{r}\right)=\text { constant }
$$

Assuming that the resident concentration and its gradient will be zero at infinite distance $(x \rightarrow \infty)$ from the inlet $(x=0)$; this leads to the common boundary condition

$$
\lim _{x \rightarrow \infty} \frac{\partial C_{r}(x, t)}{\partial x}=0 .
$$

This boundary condition does not influence concentrations within the domain for a semiinfinite system. 
These zero-concentration-gradient boundary conditions are also applied at the exit boundaries of finite domains of length $L$, because concentration is assumed to be continuous across the exit for a finite domain and there is no dispersion beyond the exit (van Genuchten and Wierenga, 1986):

$$
\left.\frac{\partial C_{r}(L, t)}{\partial x}\right|_{x=L}=0 .
$$

These zero-concentration-gradient boundaries allow advective flux of solute but prohibit diffusive or dispersive fluxes. When a zero-concentration-gradient boundary condition is applied to Equation (44), the resident concentration and flux average concentration become equal at the boundary.

An initial condition must be provided to solve the governing equation (47). The initial condition represents the distribution of concentration in the domain at $t=0$ or at the beginning of simulation. Depending upon the physical situation, different initial conditions can be designed, e.g. uniform, point, non-point, or solute-free domain. In the problem considered below, the medium is assumed free of solute at time $t=0$, which can be expressed as follows:

$$
C(x>0,0)=0 .
$$

The above expression shows that the medium is solute free except at the boundary at time $t=0$, because the boundary condition $C(0, t>0)=1$ is also implemented at time $t=0$.

\subsection{Analytical solutions}

Table 1 shows four analytical solutions for the one-dimensional advectiondispersion equation (ADE) that correspond to first- and third-type inlet boundary 
conditions for finite and semi-infinite domains. The LB model is verified against these four analytical solutions for diffusion as well as dispersion problems. The four solutions differ significantly at small Peclet number reflecting the different boundary conditions (van Genuchten and Wierenga, 1986). 


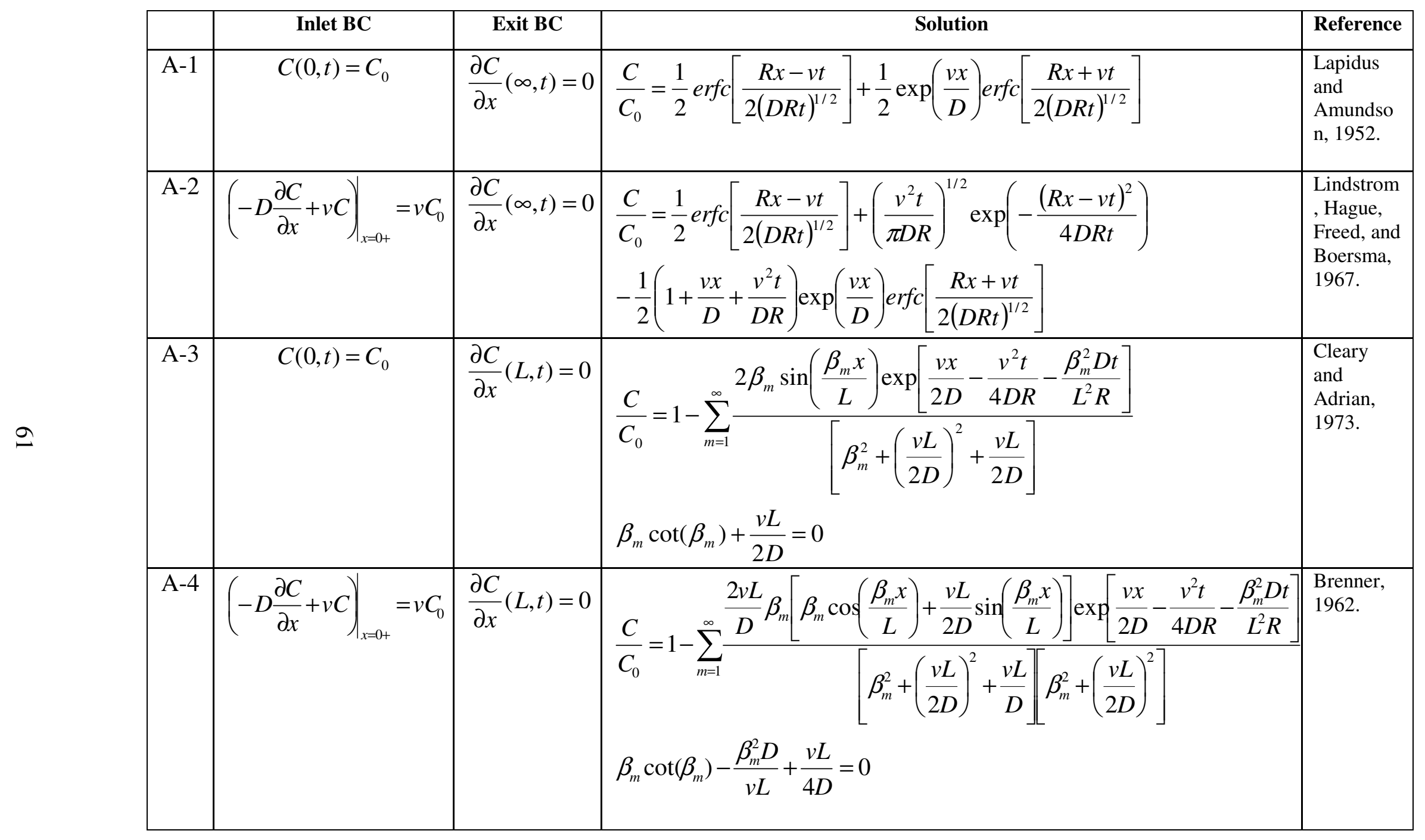

Table 1. Analytical solution of advection-dispersion equation for different boundary condition (van Genuchten and Wierenga, 1986). 


\subsection{Results and Discussion}

This section presents validation of LBM simulations of solute transport against one- and two-dimensional analytical solutions for the entire range of Peclet numbers. This study reiterates the importance of boundary conditions on resultant breakthrough curves and demonstrates the ability of the LBM to simulate such boundary value problems. The flow in these simulations is always periodic in the longitudinal direction; however concentration boundaries are applied at the inlet and exit sections for the solute component. A No-slip condition for both fluid and solute is implemented on the solid nodes (parallel walls of the slit) using a standard bounce-back approach.

First, the breakthrough curve for zero-diffusion transport in a two-dimensional parallel-wall slit is computed using the LBM and compared with the analytical solution. Then diffusion at a moving solute front is simulated for the different combination of boundary conditions and domain length. Finally, Taylor dispersion problems are simulated for the same combinations and compared with the appropriate analytical solutions.

For LB simulation of the fracture, conduit and karst systems, the $P e$ number is used to calculate the LBM parameters a priori. In the LBM simulations, the maximum velocity $u_{0} \approx 0.1 \mathrm{lu}^{-1} \mathrm{~s}^{-1}$ is often fixed to remain consistent with the low Mach number (ratio between the speed of an object and the speed of sound) assumption involved in the derivation of the Navier-Stokes equations from the LBM. The kinematic viscosity $v$ is computed from the relaxation parameter $\tau$ for the solvent component using equation (9). 
Similarly a relaxation time $\tau_{B}$ for the solute component is related to the diffusion coefficient as shown in equation (11). If gravity (body force) is used as a driving force for flow, then, after setting the maximum velocity $u_{0}$ and choosing a viscosity a priori for the simulations, calculation of the required body force for flow in a slit of width $2 a$ follows from Poiseuille's law as (Tritton, 1988):

$$
g=\frac{2 u_{0} v}{a^{2}} .
$$

The average velocity $u$ in a slit is related to the maximum velocity $u_{0}$ as follows:

$$
u=\frac{2}{3} u_{0} .
$$

The desired Peclet number $(P e)$, molecular diffusion coefficient, and average velocity are used to find the distance from the inlet at which the breakthrough curve will be measured in the one-dimensional domain:

$$
L=\frac{P e D_{m}}{u} .
$$

Note that this $P e$ is scaled by the length of the transport domain rather than by the pore length as is usual in many contexts.

\subsubsection{Zero-diffusion transport}

Breakthrough curves computed for transport in a slit or tube in the absence of diffusion using Equations (36) or (37) are fundamentally different from those computed with the advection-dispersion model. Due to laminar flow conditions and the absence of diffusion/dispersion, the solute front does not spread over the width of the channel, but follows the streamlines and creates a parabolic front as shown in Figure 17. 


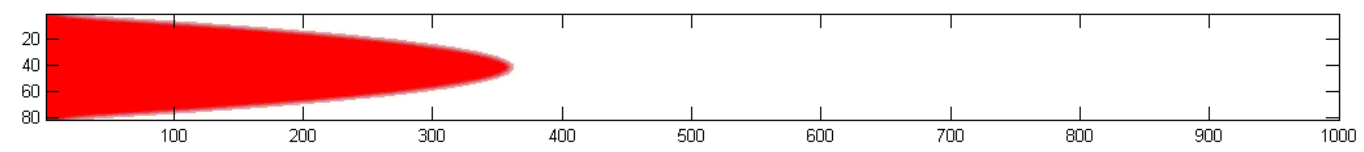

Figure 17. Solute front for zero diffusion with uniform flow in a parallel slit $(1000 l u \times 80 l u)$.

Under these conditions, there is a sharp rise in the relative concentration when the solute front reaches the cross-section where concentration is measured (Figure 18). Then the breakthrough curve levels asymptotically and the relative concentration approaches 1 at infinite pore volumes as expected from (36). LBM simulation of transport with low diffusion under Poiseuille flow shows a very good match with the analytical solution for zero diffusion transport between a pair of parallel plates. This is a limiting case of solute transport at very high Peclet number, which the LBM could successfully simulate.

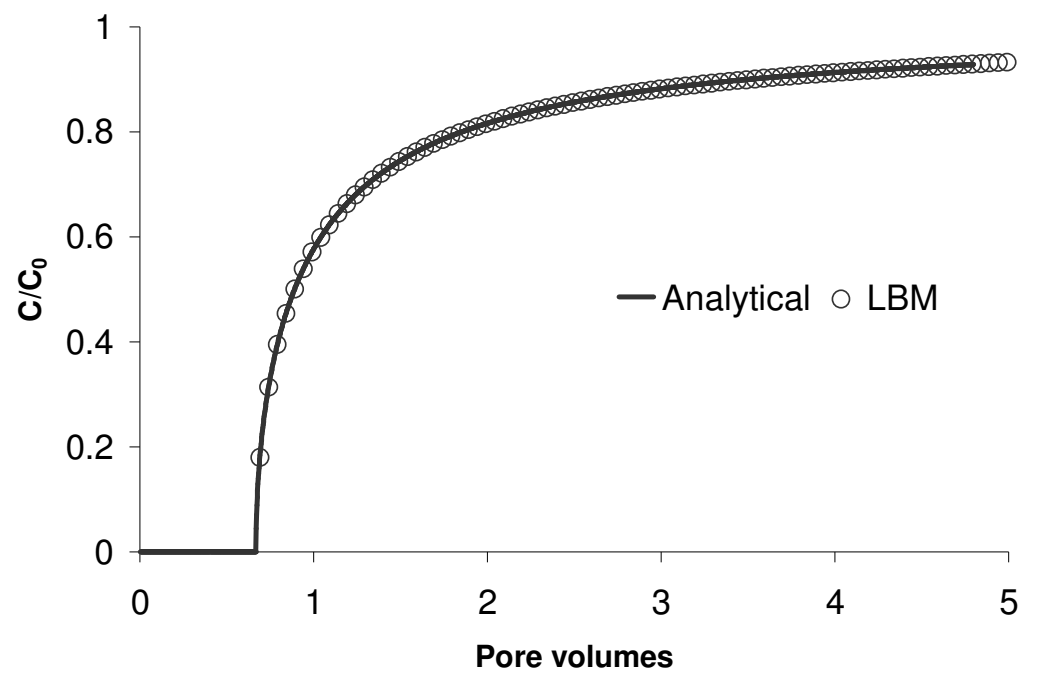

Figure 18. Breakthrough curve for zero diffusion in a slit at distance $L=100 l u$ from inlet. The slit width $2 a$ is $80 l u$, the average velocity is $0.02 l u-t s^{-1}$ the kinematic viscosity is $0.1666 l u^{2} t s^{-1}$ and the simulation's diffusion coefficient is $0.000333 u^{2} t s^{-1}$, leading to a Peclet number $P e=6667(P e \rightarrow \infty)$. 


\subsubsection{One-dimensional Advection-Diffusion Problem}

This section presents a comparison of four LBM solutions, which employ all combinations of the first- and third-type inlet boundary conditions for finite and semiinfinite domains, with the appropriate analytical solutions from Table 1. For smaller Peclet number $(<20)$, the inlet boundary condition has a strong influence on breakthrough curves measured in finite and semi-infinite domains.

The LBM simulation is conducted in a $1000 l u \times 25 l u$ domain with uniform flow condition that makes the problem effectively one-dimensional. The solute is carried as a uniform front subjected to diffusion. The effluent breakthrough curve for resident concentration is measured at $25 \mathrm{lu}$ (out of $1000 \mathrm{lu}$ ) from inlet boundary to make the domain effectively semi-infinite. Two different simulations were made in this domain for first- and third-type inlet boundary conditions in the semi-infinite domain corresponding to solutions A1 and A2 of Table 1. The longitudinal velocity $u$ is $0.0066 l u t s^{-1}$, the diffusion coefficient $D_{m}$ is $0.1666 l u^{2} t s^{-1}$, and the kinematic viscosity $v$ is $0.1666 l u^{2} t s^{-1}$. The LBM solutions are shown as open symbols and analytical solutions are represented as solid lines. The Peclet number is 1 and the analytical solutions A1 and A2 do not match each other as shown in Figure 19. Another pair of simulations was run for same flow and transport parameters $(P e=1)$ in a finite domain $(25 l u \times 25 l u)$ and resident concentration breakthrough was measured after every $250 \mathrm{ts}$ at $25 \mathrm{lu}$ from the inlet section. This gave another pair of breakthrough curves, A3 and A4, as shown in Figure 19. Again, the breakthrough curves showed a good match with their corresponding analytical solutions. 


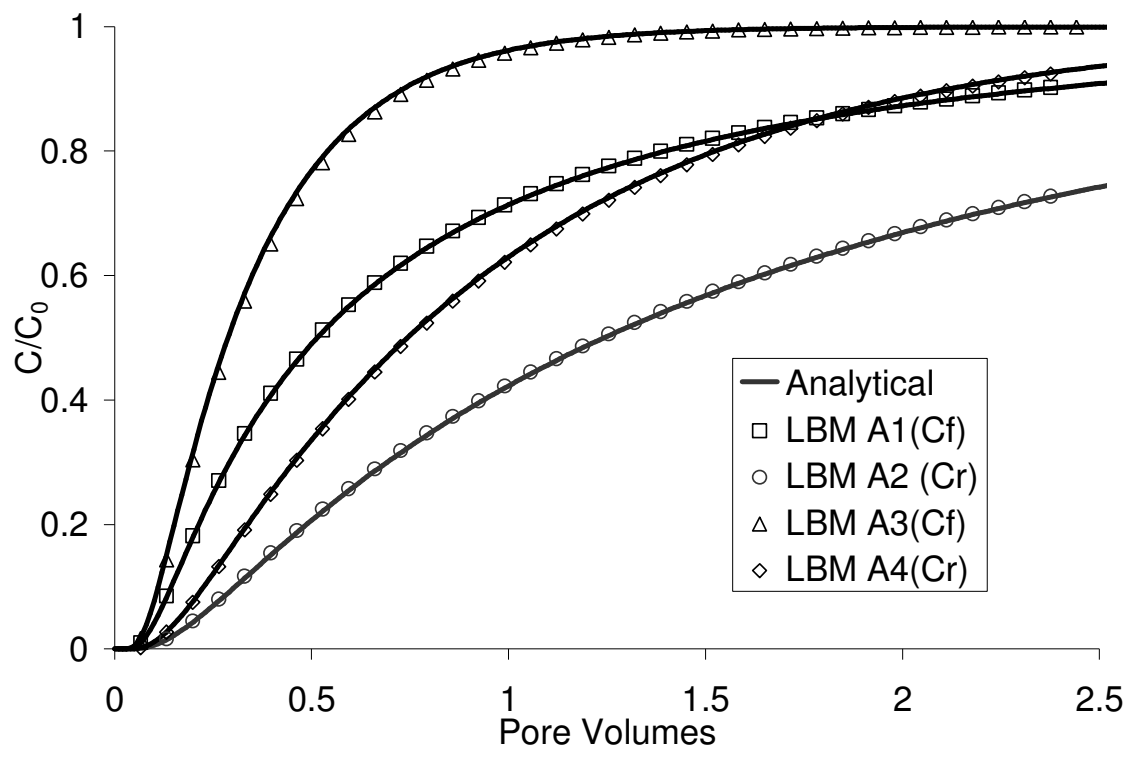

Figure 19. Breakthrough curves for four different set of inlet and outlet boundary conditions at $P e=$ 1. $C_{f}$ is flux averaged concentration and $C_{r}$ is resident concentration. A1, A2, A3, and A4 are defined in Table 1.

\subsubsection{Taylor-dispersion problem}

The above results were obtained for effectively one-dimensional finite and semiinfinite domains with first-type and third-type inlet boundaries and zero-concentrationgradient exit boundaries. In order to simulate the Taylor problem, the flow is pressure driven by a density gradient set across the domain. The flow moves between the slit's parallel walls, which impose a Poiseuille velocity profile. The length of domain is 1000 $l u$ and width is $20 l u$ and flow and transport parameters are the same as in the onedimensional simulations to achieve $P e=1$. The effluent concentration is measured $100 l u$ from the inlet once every $250 t s$ to produce the breakthrough curve. Figure 20 shows results of the LBM simulations compared against the analytical solutions shown in Table 1. The LBM solutions (open symbols) show good agreement with the analytical solutions (solid line) for first- and third-type inlet boundaries. 


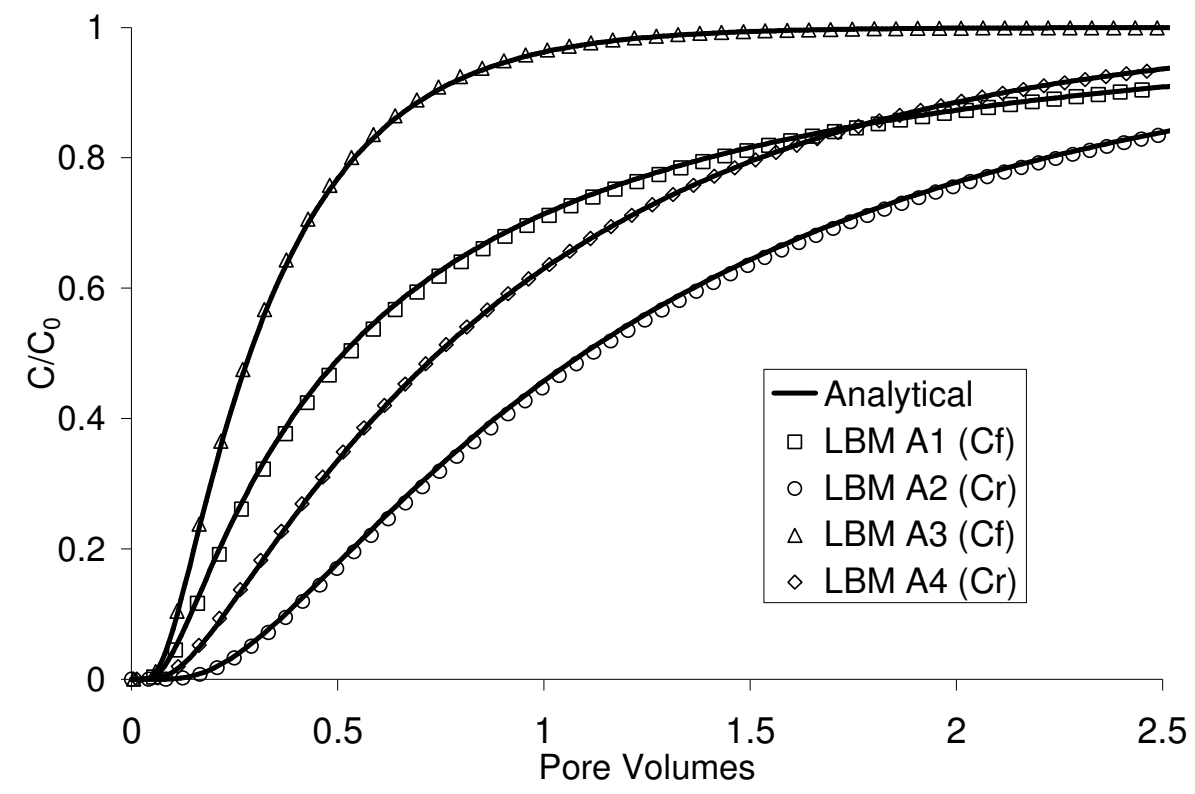

Figure 20. Breakthrough curve at $P e=1$ for transport in parallel slit for four different combination of inlet boundary conditions and domain type.

These results demonstrate that the LBM can simulate Taylor dispersion for varying inlet boundary conditions and domain lengths considered by the four onedimensional analytical solutions. For higher Peclet number, the breakthrough curve will have a significantly different shape as shown in Figure 21. In this simulation, the domain is $10000 l u \times 32 l u$, the molecular diffusion coefficient is $0.005 l u^{2} t s^{-1}$, and the effluent concentration is measured at 10,000 $l u$ from the inlet section. The average flow velocity $u$ $=0.02 \mathrm{lu} \mathrm{ts}^{-1}$. Thus $P e=120$. The predicted dispersion coefficient following equation (40) is $0.349 l u^{2} t s^{-1}$, and the fitted dispersion coefficient is $0.376 l u^{2} t s^{-1}$. 


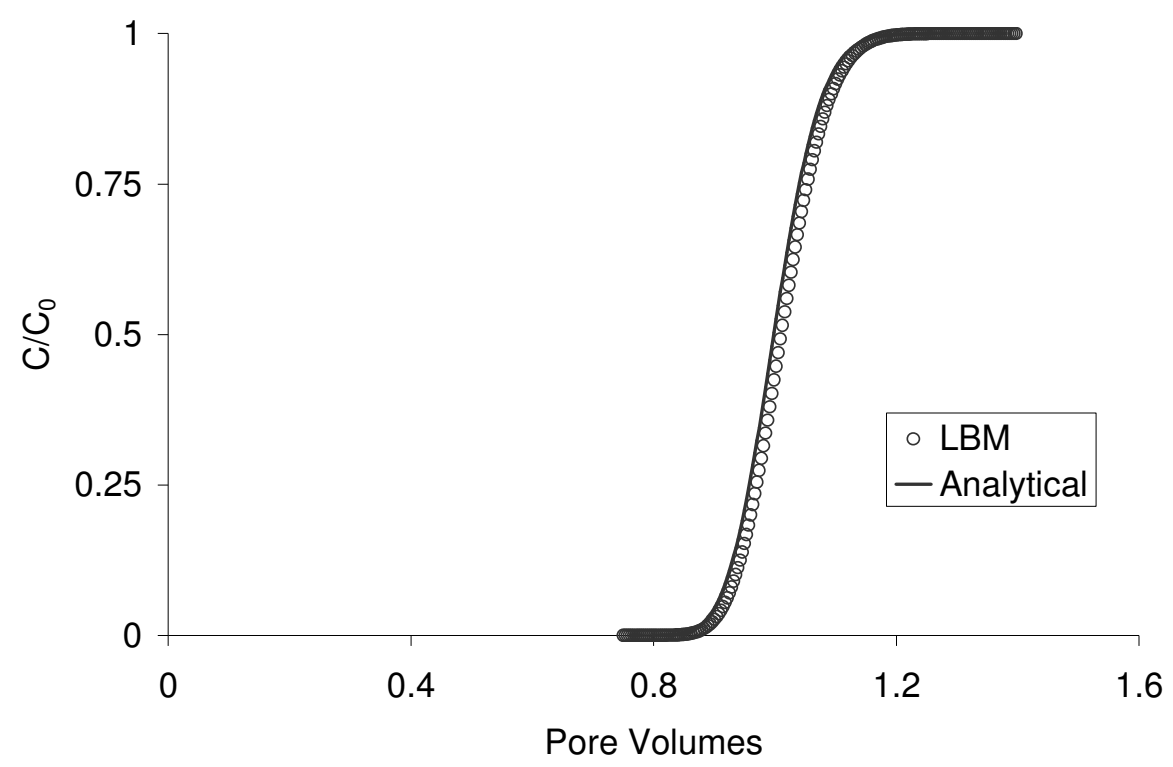

Figure 21. Breakthrough curve for flow in a slit at $P e=120$

This has proven the LBM's ability to simulate solute transport for different inlet boundary conditions in finite and semi-infinite domains. Table 1 shows four different sets of analytical solutions for effluent breakthrough curves. The sensitivity of effluent breakthrough curves to different inlet boundary conditions and domain type is observed only at smaller Peclet number $(\mathrm{Pe}<20)$. This was chosen as a robust test for the LBM to verify its ability to simulate solute transport controlled by inlet boundary condition for solute and the type of domain. The LBM was able to simulate the apparent dissimilarity in breakthrough curves for different inlet types and domain types at smaller Peclet number. This shows that solute transport is governed by inlet boundary conditions for solute at small Peclet numbers, which can be considered as one extreme of the solute transport regime. Another extreme of solute transport regime is considered to be a transport process at practically infinite Peclet numbers. There are two different ways to 
achieve infinite Peclet number: by making the carrier fluid velocity very high or by making the diffusive transport extremely small or negligible. The LBM puts a limitation on having too high a velocity, so the diffusion coefficient is pushed to near its lower limit to achieve near-zero diffusive transport. In a slit, solute follows a parabolic profile identical to the velocity profile when there is zero diffusion across the front. The effluent breakthrough curve exhibits a sudden rising limb when the front hits the observation section and then grows asymptotically towards complete breakthrough. The simulated effluent breakthrough curve for zero-diffusion transport showed a good fit to the analytical solution. The results of this chapter verify the ability of the LBM to solve solute transport in all possible ranges of Peclet number for different boundary conditions. Hence, the method is suitable for application to solving physical problems in large-scale karst aquifers. 


\section{SOLUTE TRANSPORT IN KARST AQUIFERS}

Modeling flow and transport in karst aquifers is a frontier challenge for ground water science. In phreatic karst, the challenges arise from three sources: first, the conduits that characterize karst aquifers are exceedingly hard to delineate; second, standard Darcy's law-based approaches can not address the inertially-dominated flows and resulting eddy mixing that occur in karst conduits; and third, critical transport between conduits and surrounding aquifer based on simple capacitance-based 'matrix diffusion' models is not adequate for simulation of transport in many circumstances. The ability to delineate conduits and quantify their geometry is improving due to advances in geophysical techniques. In addition, karst aquifers and their conduits may be amenable to geostatistical quantification and good progress has been made in explicit simulation of their genesis and evolution (e.g., Birk et al. 2005). This discussion focuses on the second and third challenges and introduces relatively new modeling techniques that address inertial flows, eddy mixing, and matrix diffusion. The discussion also illustrates potential applications of the techniques to karst aquifers by demonstrating capabilities for simple problems. Sukop et al. (2008) illustrate some additional applications of the new techniques to karst aquifers. Other challenges presented by transient hydraulics (Zhou, 2007; Anwar and Sukop, 2008; and Chapter 6), partial saturation, and chemical reaction (Ginzburg, 2005; Kang. et. al., 2002) might be successfully treated by the new methods in the future. 
Like fractured rock aquifers, karst aquifers are generally viewed as double- or triple porosity systems, where a porous matrix of carbonate rock with pores on the order of millimeters or less, contains macropores (roughly centimeter-scale), and may be cut by conduits ranging in size from tens of centimeters to tens of meters (White, 2002). Standard Darcy's Law-based ground water models such as MODFLOW are reliable tools for modeling slow moving (laminar regime) flow in porous media. Efforts to apply such models in karst aquifers are numerous and meet varying degrees of success. In some instances, an equivalent porous medium (EPM) model of a karst aquifer may be adequate. For example, Scanlon et al. (2003) used MODFLOW to simulate groundwater hydraulics in the Barton Springs portion of the karstic Edwards Aquifer in Texas. They applied MODFLOW with spatially distributed hydraulic conductivity on approximately a 20-km scale and showed good agreement between measured and simulated discharge at Barton springs. This has been referred to as a 'smeared conduit' model by Green et al. (2006). EPM models are expected to perform better at large scale where the effects of many conduits can be integrated (Huntoon, 1995).

A more elaborate approach is to incorporate conduits explicitly ('embedded discrete-feature' models of Green et al. (2006)). Finite element-based models (e.g., FEFLOW) are better able to incorporate discrete features such as conduits in karst aquifers. The accuracy of this method depends upon grid resolution, and algorithms for grid refinement and grid adaptation are available. Even though it should be feasible to solve the Navier-Stokes equations for inertial flow in complex discrete features such as conduits with finite-element models, this does not seem to be widely available to the 
ground water community at this time. For example FEFLOW (Diersch, 2002) uses the Darcy, Hagen-Poiseuille, or Manning-Strickler laws to simulate flow in discrete elements. The first two are strictly applicable for laminar flow and the third is based on straight sections of uniformly sloping conduit with simple cross-section and a roughness parameter.

In the terminology of Green et al. (2006), the dual-conductivity model (DCM) approach is an alternative to single continuum approaches. This method is closely related to the fissured rock/double porosity model first developed for fractured rock by Barenblatt et al. (1960). In these models, a network of conduits can be coupled to a continuum representation of the matrix rock or, if the conduit network is dense, it can also be modeled as a separate continuum. Linkage between conduit and matrix is achieved through linear exchange coefficients. Sun et al. (2005) presented a dualconductivity model called MODFLOW-DCM. The flow regime in the conduits is either Darcy (laminar) or Darcy-Weisbach (turbulent) (Sun et al. 2005).

The CAVE (Carbonate Aquifer Void Evolution) model (Clemens et al. 1997) is a model where flow in a conduit network is calculated using Kirchhoff's law together with the Darcy-Weisbach equation to relate discharge with head difference. The ColebrookWhite equation is used for estimation of the friction factor. The CAVE model also predicts the dissolution enlargement of pores or conduits. Birk et al. (2005) integrated the equilibrium and non-equilibrium (two-region) Advection-Dispersion Equation (ADE) with CAVE and compared field spring discharge and tracer breakthrough data with 
model predictions. The two-region model fit observed breakthrough better than the advection-dispersion model, but both models failed to capture the long tailing.

Shoemaker et al. (2008) added a module called Conduit Flow Process Mode 2 (CFPM2) to MODFLOW to simulate inertial flow in karst aquifers. The ground water flow equation is solved and the laminar hydraulic conductivity is replaced by turbulent hydraulic conductivity when the head difference between cells (computed by MODFLOW) exceeds a critical head difference. Laminar and turbulent hydraulic conductivity are linked by an empirical factor, which is a function of the square root of the ratio between critical head difference and actual head difference. This develops a nonlinear relation between discharge and hydraulic gradient under turbulent conditions. Turbulent flow was thought to be widespread in preferential flow layers of the Biscayne aquifer. This type of flow modeling approach in conduit/matrix systems may estimate the discharge rate to some acceptable agreement, but the local characteristics of inertial flow like eddies can not be accounted for. Solute transport is strongly influenced by such eddies on some time scales. Solute is trapped in vortices and slowly diffused back out to the surrounding flow system.

Two-region models have been applied in the past to predict tracer breakthrough in karst aquifers. QTRACER (Field, 1999) is an inverse modeling program used to estimate hydraulic and transport parameters from tracer breakthrough curves. Hydraulic properties and transport parameters are estimated from the shape of the breakthrough curve using the method of moments. Aquifer geometric properties are estimated by evaluating 
discharge with respect to residence time. Solute transport capabilities of this model are based on the two-region approach. Field and Pinsky (2000) applied the two-region model to simulate skewness of breakthrough curves obtained from karst aquifers. The tworegion model was found to fit the strong tailing effect better than the advection-dispersion model. Inability to estimate some model parameters a priori can make model parameter sets non-unique. Massei et al. (2006) compared QTRACER and a linear graphical method to simulate the tracer breakthrough obtained from a karstic aquifer. Birk et al. (2005) compared the moment analysis method, two analytical models (one two-region based), and a numerical model to interpret tracer breakthrough curves in carbonate aquifers. The two-region model was found to be better at fitting long tails than all other models. Geyer et al. (2007) used the two-region model in CXTFIT (Toride et. al., 1999) to calibrate the tracer data obtained from a large-scale multi-tracer test in a karst aquifer. The conservative tracer data were evaluated to estimate model parameters subsequently used for reactive tracer transport. The authors concluded that this method removed ambiguity in parameter set identification, though the model failed to predict tailing behavior. The model was found to be insensitive to dispersion and mass transfer coefficients. Bai et al. (1999) extended the 1-D two-region model to a semi-analytical, two-dimensional capacitance model for solute transport. This model was found to be useful for modeling anisotropic dispersion in heterogeneous porous media with two-region effects.

The Lattice Boltzmann Method has been under development by the physics community for the last several decades but applications to ground water science are in their infancy. Their potential for simulation of flow and transport in karst aquifers has 
been recognized for some time. For example, Watson et al. (2003) applied a LBM model to simulate the flow field obtained from a laboratory-scale dye tracer test. The model successfully simulated distinct recirculation zones observed in the laboratory karst analogue system. In part, the slow transference of LBMs to ground water applications is

due to the variety of processes and scales that a viable ground water flow and transport model—especially one that simulates inertial flows and eddy mixing-must incorporate and address. LBMs for these purposes continue to improve rapidly and it is expected that fully-functional models comparable to FEFLOW and MODFLOW/MT3D may be available within five years or less. A key advantage of this next generation of the LBM models is that they will retain the ability to solve inertial flows and eddy mixing needed for karst aquifers and do it at field scales.

\subsection{Example applications to karst flow and transport processes}

The results of LB simulations relevant to processes expected in karst aquifers are summarized in this section. The LB model is applied to simulate two-region type breakthrough, transport in heterogeneous porous media, and transport in a heterogeneous porous media traversed by a conduit with inertial flow.

\subsection{Two-region solute transport model}

Quantification of flow and transport parameters is essential for accurate prediction of solute breakthrough in karst aquifers. Several continuum (or equivalent porous medium approach) and discrete models are available to model flow in fractured and karst aquifers. Flow patterns in karst aquifers follow preferential pathways and laboratory experiments show that the breakthrough curve from such a medium generally has three 
characteristic features: a sudden rising limb, a central plateau, and a long tail (van Genuchten and Wierenga, 1986). The sudden rising limb is due to the conduit region and the long-tail is due to slow diffusion from the matrix region.

Coats and Smith (1964) proposed a mobile-immobile (dual-domain or tworegion) model to simulate transport with preferential pathways, which was later applied to laboratory column experiment data (van Genuchten and Wierenga, 1986) and field data (Brown and Ford 1971; Atkinson et al. 1973; Mull et al. 1988; Toride and Leji 1996; Field and Pinsky 2000; Maloszweski et al. 2002; Massei et al. 2006; Geyer et al. 2007; Göppert and Goldshceider 2008), and incorporated into standard solute transport codes such as the popular MT3D (Zheng, 2006). These multi-region or mobile-immobile water models are predicated on the notion of solute in mobile fluid in a fracture or conduit interacting with an immobile fluid phase in the surrounding porous matrix according to a first-order kinetic rate constant and a concentration difference. Such 'matrix diffusion' models are intuitively appealing, analytically tractable, and adequate for certain purposes, but suffer from the limitation that the matrix region is not explicitly considered but is simply a capacitance.

Advection and dispersion are the dominant mechanisms of transport in the mobile zone, whereas water in the immobile zone is linked to water in the mobile zone by diffusive transport only. The non-dimensional form of governing equations for such a two-region non-reactive transport model is (Field and Pinsky, 2000) 


$$
\beta \frac{\partial C_{m}}{\partial T}+\omega\left(C_{m}-C_{i m}\right)+\frac{\partial C_{m}}{\partial X}=\frac{1}{P e} \frac{\partial^{2} C_{m}}{\partial X^{2}}
$$

and

$$
(1-\beta) \frac{\partial C_{i m}}{\partial T}=\omega\left(C_{m}-C_{i m}\right)
$$

with non-dimensional parameters

$$
X=\frac{x}{l}, T=\frac{q t}{l}, \beta=\frac{\theta_{m}}{\theta}, P e=\frac{q l}{D}, \text { and } \omega=\frac{\lambda l}{q},
$$

where $C_{m}$ and $C_{i m}$ are solute concentration $\left(M L^{-3}\right)$ in the mobile and immobile zones respectively, $D\left(L^{2} T^{-1}\right)$ is the dispersion coefficient in the mobile region, and $\lambda\left(T^{-1}\right)$ is a first-order rate constant that controls the exchange of solute between the mobile and immobile zones. Darcy flux is represented as, $q=v \theta=v_{\mathrm{m}} \theta_{\mathrm{m}}$, where $\theta$ is the volumetric water content and $\theta_{\mathrm{m}}$ is volumetric mobile water content. $\theta$ can vary between 0 and 1 and $\theta_{\mathrm{m}}$ varies between 0 and $\theta . T$ is pore volume. There are essentially three parameters in the dimensionless two-region model: Peclet number, fractional mobile region $(\beta)$ for conservative solute, and $\omega$, which controls the mass exchange between the mobile and immobile regions. Significant efforts have been made to independently estimate these parameters, but this has remained elusive. In reality and in the LBM, matrix invasion by solute will depend upon the thickness of the matrix and the diffusion coefficient of solute therein.

In this section, LBM breakthrough simulations from a simple conduit/matrix system, representing an analytically-tractable prototype of what might be found in a karst 
aquifer, are fitted with two-region and standard advection-dispersion models. The solute transport simulation is set up in a $50 l u$ long and $25 l u$ wide domain with periodic flow boundaries on all sides as shown in Figure 22.

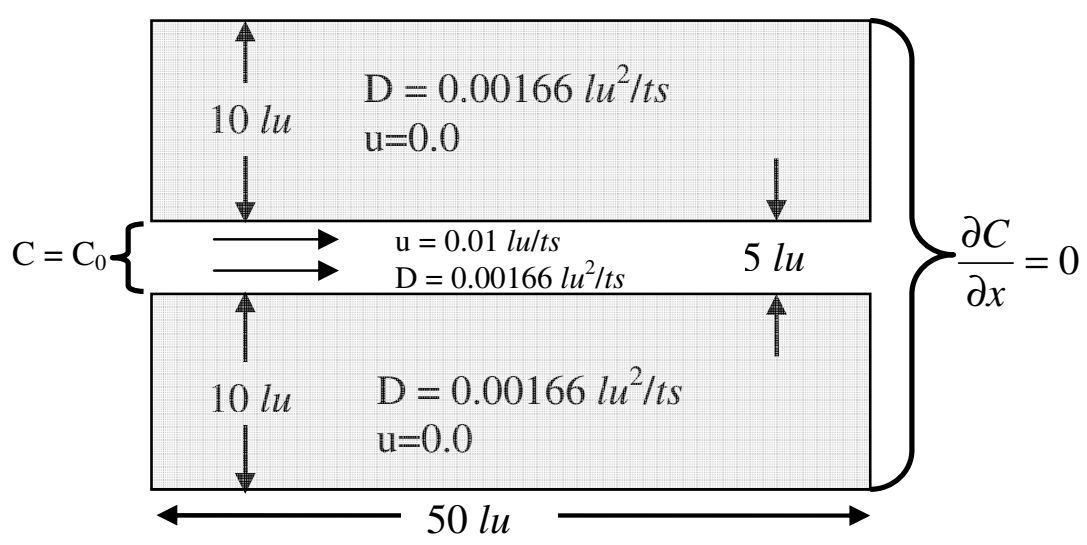

Figure 22. Schematic diagram of two-region model.

The domain has a mobile (fracture) region of thickness $5 l u$ in the center with an adjoining immobile (matrix) region. The LBM had to be constrained to match the tworegion assumptions by turning off the LBM flow model and explicitly setting zero and non-zero fluxes in the immobile and mobile regions respectively. In the two-region model, the immobile region (matrix) is effectively infinitely thin so that solute could instantly fill and continue to diffuse from the fracture (mobile) into the matrix (immobile) without regard for a reduced diffusive gradient in the matrix as the concentration in the matrix grows. 
Advection and diffusion drive solute movement in the mobile region. A constant concentration boundary is set only at the entrance of mobile region and a zeroconcentration-gradient boundary is set over the whole width of the domain on the opposite boundary. A uniform flux of $u=0.01 \mathrm{lu} / \mathrm{ts}$ is set in the mobile region.

Figure 23 shows effluent breakthrough curves from LBM simulations that have typical two-region characteristics. The two simulation results for the same Peclet number and $\omega$ but different mobile fractions $\beta(=0.2$ and 0.4$)$ are shown in Figure 23.

Breakthrough curves are fitted to the two-region model with two fitting parameters: dispersion coefficient and mass exchange rate between the mobile and immobile regions. The known flux and $\beta$ are kept fixed during the fitting. The fixed flux is equal to $\beta$ (0.2 or $0.4)$ times the actual flux $(0.01 \mathrm{lu} / \mathrm{ts})$ in the mobile region because it represents a macroscopic flux from the entire domain. The dimensionless rate constant $\omega$ is probably related to the diffusion coefficient $D$ for thin matrix zones and or short input pulses, but its physical meaning is less clear when the matrix zone is thick or the pulse is long. 


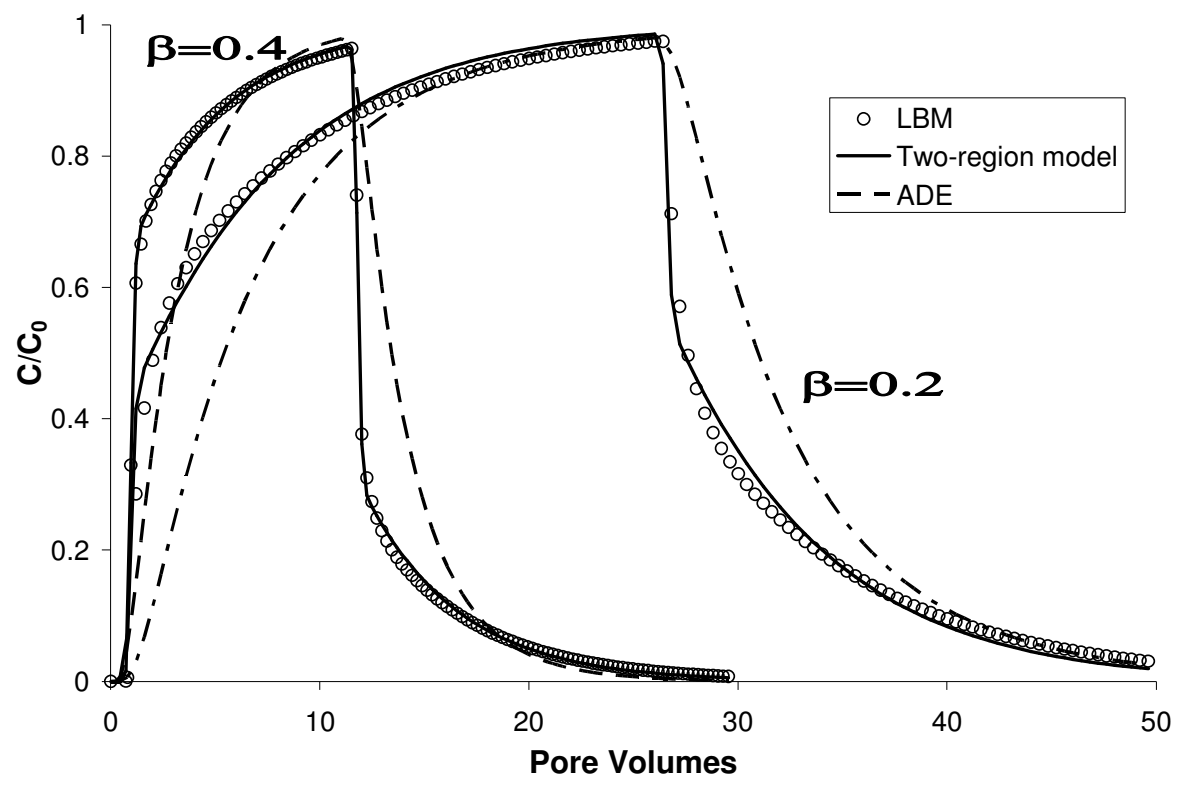

Figure 23. LB simulation fitted with two-region model and advection-dispersion (ADE) model for $\beta=0.2$ and $\beta=0.4$.

The fitted diffusion coefficient is of same order as provided in the simulation and showed low correlation $(-0.151)$ with the mass exchange rate $(\omega)$ indicating independence of the fitting parameters and uniqueness of the fits. The coefficient of determination $\left(R^{2}\right)$ is above $99.9 \%$ for each case. The breakthrough curve shows a poor fit with the standard advection-dispersion (ADE) model, having $R^{2}=85 \%$ for $\beta=0.2$ and 0.4. These results are obtained using a constrained approach where velocity fields are explicitly set in the matrix and conduit regions. 


\begin{tabular}{|l|l|l|l|l|}
\hline Parameters & $u(l u / t s)$ & $\omega$ & $D_{m}\left(l u^{2} / t s\right)$ & $R$-square \\
\hline LBM & 0.004 & Not applicable & 0.00166 & not applicable \\
\hline Two-region & 0.004 & 0.411 & 0.00212 & 99.96 \\
\hline ADE fit & 0.004 & Not applicable & 0.0739 & 85.21 \\
\hline
\end{tabular}

Table 2. Model parameters for $\beta=0.4$.

Martin and Screaton (2001) asserted that karst aquifers can not be considered to have purely diffusive or purely conduit flow but rather a mixture of two types of flow. They believed flow in matrix region is laminar whereas flow in conduit is turbulent and solute transport is directly influenced by the flow conditions in matrix and conduits. Younger carbonate aquifers such as Tertiary carbonate platform of Florida, Yucatán, and many ocean islands have high matrix porosity and permeability (Martin and Screaton, 2001). They cited several examples from the literature where significant exchange between conduit and matrix is observed. Hence, better understanding of exchange between matrix and conduit regions is necessary for water resource management and aquifer remediation approaches in carbonate aquifers.

An example of matrix-conduit interaction in a system of such relatively high matrix permeability is presented below, where velocity fields are solved in the conduit with standard Navier-Stokes LBM, and in the matrix using the LBM-based, macroscopic Darcy flow solver. The domain is similar to the one shown in Figure 22: $40 \mathrm{lu}$ wide and $20 l u$ long with a $10 l u$ wide conduit in the center. The flow is pressure driven with a 
pressure difference of $3.333 \times 10^{-5} \mathrm{mu} / \mathrm{lu} \mathrm{u}^{1}-t \mathrm{~s}^{2}$ between the left and right boundaries. The relaxation parameters for fluid and solute components are $0.51 \mathrm{ts}$. The solute breakthrough curve shown in Figure 24 is measured at the end of the domain, $20 l u$ from the inlet boundary. A constant concentration boundary across the whole width $(40 l u)$ is applied at the inlet boundary (left side) and a zero-concentration-gradient boundary is applied at the exit boundary (right side). This is in contrast to the preceding two-region LBM model where the concentration boundary is applied only at the fracture opening. Similarly, the driving force (pressure boundary condition) is also applied over the whole (left and right boundaries) to cause flow in the matrix as well as the fracture region. Nodes in the matrix region have $\mathbf{R}=0.1 \mathrm{ts}^{-1}$ and nodes in fracture region have $\mathbf{R}=0$. The permeability of each region in lattice units $(l u)$ can be calculated using equation (18). For solute transport, the diffusion or dispersion equation is solved in the conduit and matrix regions respectively. The dispersivity in the matrix region is set equal to $0.81 l u$. 


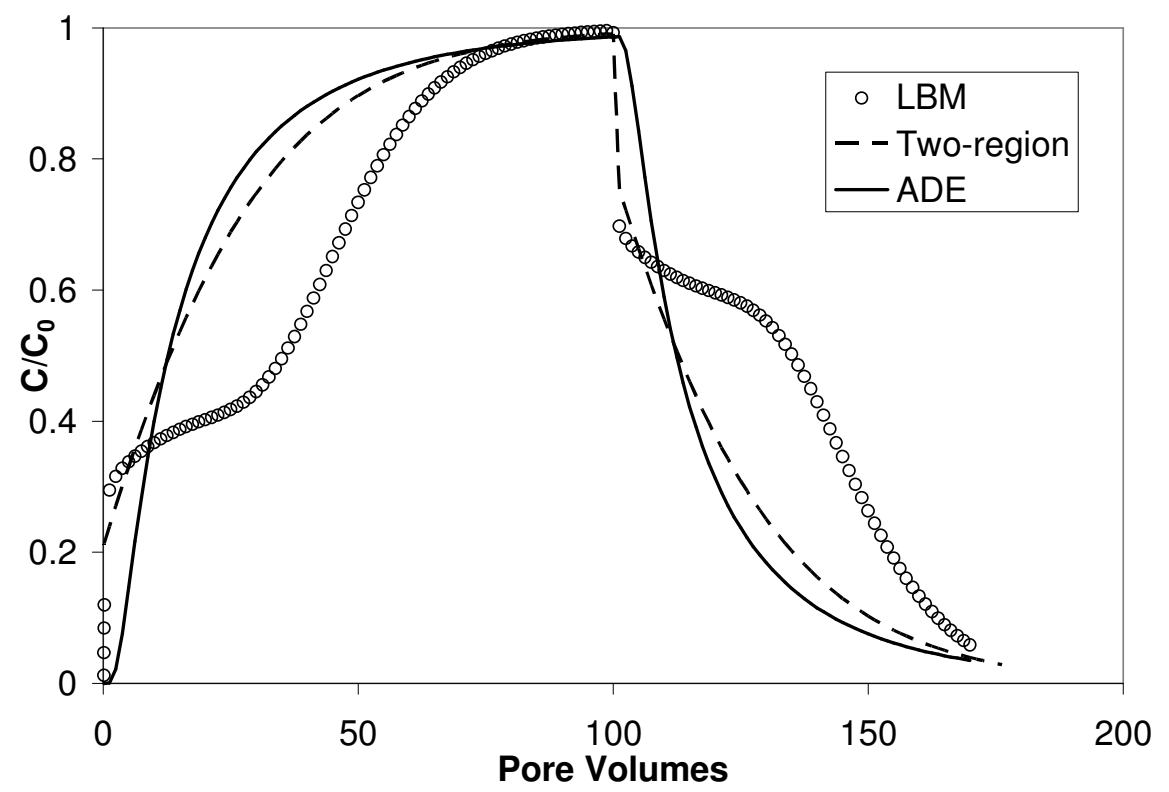

Figure 24. Solute breakthrough curve measured at the end of $20 \times 40 l u$ domain with conduit (10 lu) in the center. The breakthrough curve (open circles) obtained from the LBM is fitted with advectiondispersion equation (ADE) (solid line) and two-region (broken line) models.

The breakthrough curves in Figure 23 and Figure 24 are substantially different due to contributions from explicit simulation of the matrix region. The rise in concentration after the plateau at $\mathrm{C} / \mathrm{C}_{0}=30 \%-40 \%$ is due to direct contribution to the effluent from the matrix at the discharge face, which is not possible in the two-region paradigms; a similar trend is shown by the falling limb. The qualitative nature of the breakthrough curve from the LBM as shown in Figure 23 is similar to breakthrough curves from karst aquifers observed in field or laboratory settings (Figure 1, Figure 2 and Figure 3). It is shown in Figure 24 that neither analytical model was able to capture the complexity of the breakthrough, though the two-region model was better at fitting the rapid rise and fall. Hence, the LBM could be a potential approach to simulate breakthrough curves in karst aquifers. 


\subsection{Transport in heterogeneous porous media}

Porous media normally exhibit heterogeneous permeability (Bear, 1972; Cortis and Berkowitz, 2004; Berkowitz et. al., 2006). Combining the LBMs for heterogeneous porous media and anisotropic dispersion allows solute transport simulation of effluent breakthrough curves from such heterogeneous porous media. We illustrate this in a domain that is $81 l u \times 81 l u$ with resistance $(\mathbf{R})$ that varies from 0 to $0.05 t s^{-1}$ as shown by gray scale in Figure 25, which represents the permeability field following equation (18). Each node in this image is assumed to represent 10's to 100's of meters of scale depending upon the desired non-dimensional Darcy number, $k / L^{2}$, where $\mathrm{L}$ is a characteristic length.

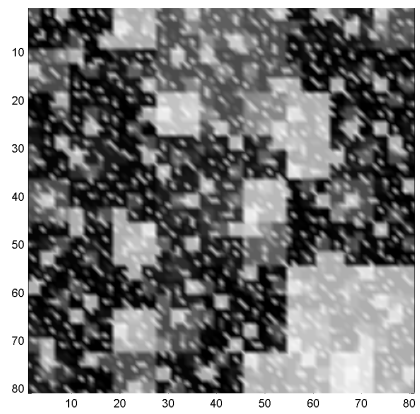

(a)

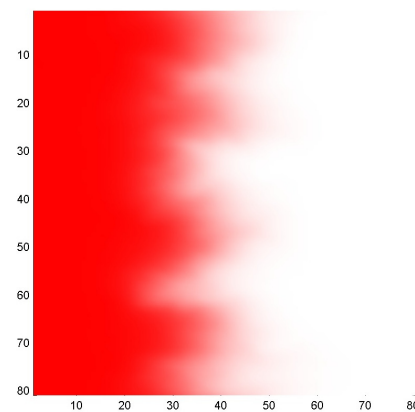

(b)

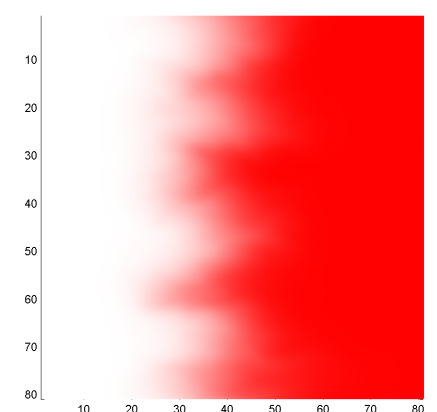

(c)

Figure 25. (a) Heterogeneous permeability field in grey scale. Lighter grey represents higher permeability. Red color is tracer and white is fluid (b) Snapshots of solute front before breakthrough and (c) during flushing of domain shown in (a). The units are in $l u$.

A pressure boundary condition (Zou and He, 1997) is applied on the left $(P=$ $\left.0.333666 \mathrm{mu} / \mathrm{lu}-\mathrm{ts} \mathrm{s}^{2}\right)$ and right boundaries $\left(P=0.33333 \mathrm{mu} / \mathrm{lu}-\mathrm{ts} \mathrm{s}^{2}\right)$, and forces flow from left to right. Solute enters the domain from the left boundary after 6,000 $t s$ and the solute pulse lasts for $280,000 t s$. The relaxation parameter $(\tau)$ for the fluid is $0.501 t s$ and $\tau_{B}=$ 
$0.51 \mathrm{ts}$ for the solute component. A zero-concentration-gradient boundary is applied to the solute component at the right boundary. Top and bottom boundaries are periodic for both the fluid and solute components. Longitudinal and transverse dispersivities are set equal to $1.1 \mathrm{lu}$ and $0.41 \mathrm{lu}$ respectively at every node. The average flow velocity is $0.000474 \mathrm{lu} / \mathrm{ts}$.

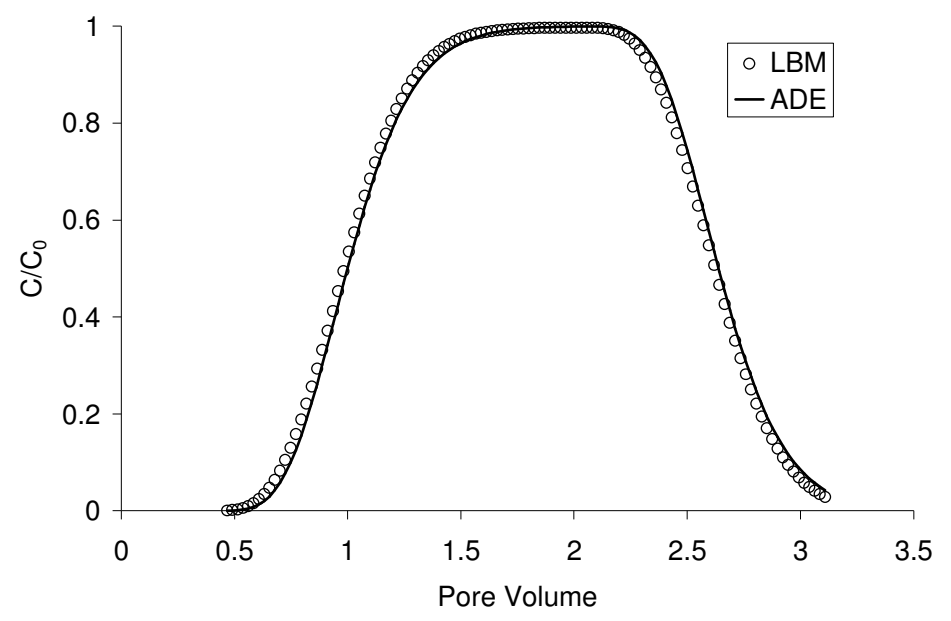

Figure 26. Effluent breakthrough for heterogeneous domain as shown in Figure 25.

A snapshot of the solute front is shown at 67,200 $t$ s after the solute enters the domain and 75,200 ts after flushing of the domain begins (Figure 25). The advected and diffused front is non-uniform due to the varying permeability field, which causes regions of fast and slow moving solute. The high permeability region (brighter color in Figure 25 a) allows quick invasion by the solute front whereas it takes much longer to cross the low permeability region (darker color in Figure 25 a). Effluent breakthrough is measured at the exit section of the domain and is shown in Figure 26. The effluent breakthrough curve is fitted to the advection-dispersion model with the flux measured as the average over the whole domain in the LBM simulation. The solute is allowed to undergo both advection 
and dispersion in porous media (matrix) contrary to traditional models which consider solute as completely immobile in matriz region; only first-order mass exchange is assumed between matrix and conduit zone.

\subsection{Solute transport for inertial flow in a conduit}

Flow in a planar channel or pipe is function of third order of radius of pipe and this is important in karst aquifers as conduit diameter ranges from few cm's to meters. Hence, the flow can get rapid and exhibit inertial features like eddies and voritices. The damping effects normally imposed on the fluid flow by small pores (say less than $1 \mathrm{~cm}$ ) that characterize many porous media usually eliminate the inertial (eddy and turbulent) aspects of flow, but, in karst aquifers, porous media are routinely traversed by largediameter conduits and this allows flow to become inertial. The widths of karst conduits (characteristic length) could be tens of meters or more, and sometimes flow within them is comparable to a free surface, turbulent river at very high flow rate. Flow in fractures becomes turbulent when the Reynolds number exceeds 500 (White, 2002), although eddies can form at much lower Reynolds number and exert a strong influence on solute transport. Flows that contain eddies are referred as 'inertial'. When flow reaches the inertial and turbulent regimes the relationship between head loss and discharge becomes non-linear and solute transport becomes more difficult to simulate due to eddy mixing, entrapment in eddies, and slow diffusion out of the eddies.

Figure $27 a$ shows an $81 l u \times 81 l u$ heterogeneous porous background with a conduit. Background permeability varies between 0.0 to $0.0068 l u^{2}$. Background 
permeability varies as shown in grey scale. The model solves Navier-Stokes-based flow (equation (4))in the conduit region, while in the porous region, it solves Darcy flux. For solute transport, anisotropic dispersion $\left(D_{L}=0.81 \mathrm{lu}\right.$ and $\left.D_{T}=0.27 \mathrm{lu}\right)$ is solved in the porous region and the advection-diffusion equation is solved in the conduit. The relaxation parameters $\tau$ for fluid and solute are $0.501 t s$ and $0.51 t s$ respectively. The flow is gravity driven at $g=1 \times 10^{-5} l u / t s^{2}$ and the maximum velocity in the conduit is 0.051 $l u / t s$. For solute, zero concentration gradient conditions are set on the top, bottom, and right boundaries. Figure $27 b$ and $c$ shows eddy mixing of solute in the conduit due to highly inertial flow. These snapshots are at $3000 \mathrm{ts}$ after solute entered the domain from the left boundary and 10,000 ts after flushing of the domain begins. The pulse duration for solute is $495,000 \mathrm{ts}$. The time step $(t s)$ can be readily converted into non-dimensional pore volumes using volume average flux $(0.003471 \mathrm{lu} / \mathrm{ts})$ and domain length $(81 \mathrm{lu})$.

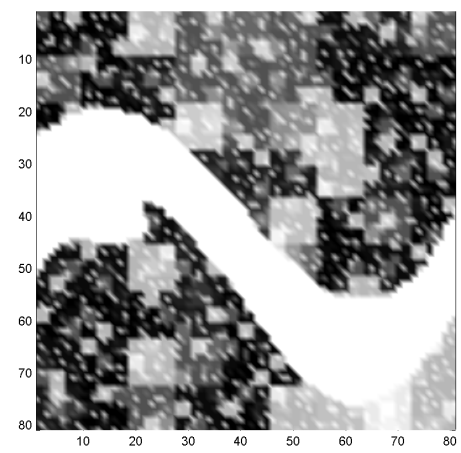

(a)

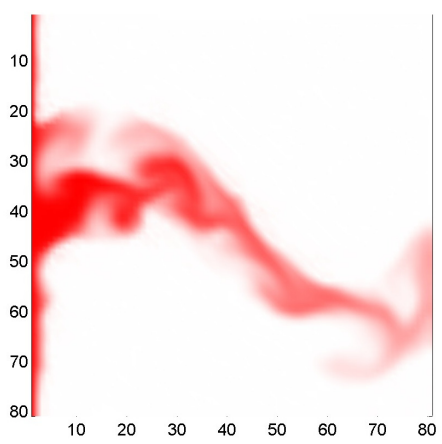

(b)

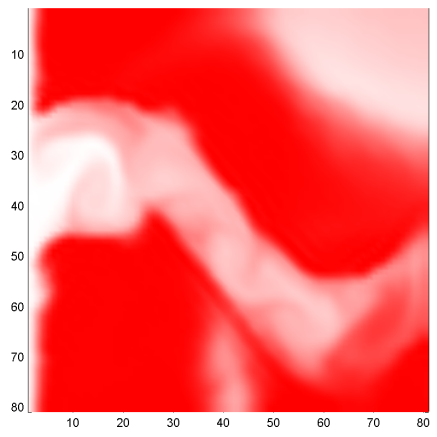

(c)

Figure 27. (a) Heterogeneous porous background with conduit. (b) Simulated snapshot of solute in domain soon after initial solute injection and (c) during flushing of domain. The units are in $l u$.

Effluent breakthrough is measured at the end of the domain and normalized with respect to inlet concentration. Figure 28 shows breakthrough at the end of the domain simulated using the LBM. The sudden rise is due to the rapid arrival of effluent from the 
conduit and a plateau is formed as solute takes much longer to travel through the background porous media. Heterogeneity in the porous medium causes early breakthrough in some regions and after that, concentration rises slowly. Behavior during flushing is similar to during loading of the domain. Flushing causes a sudden drop in the breakthrough curve as the conduit rapidly flushes out. Long tailing is observed as the solute is slowly flushed from the porous region. This type of effluent breakthrough curve is generally observed in karst-type aquifers with two distinct flow region: conduit and matrix.

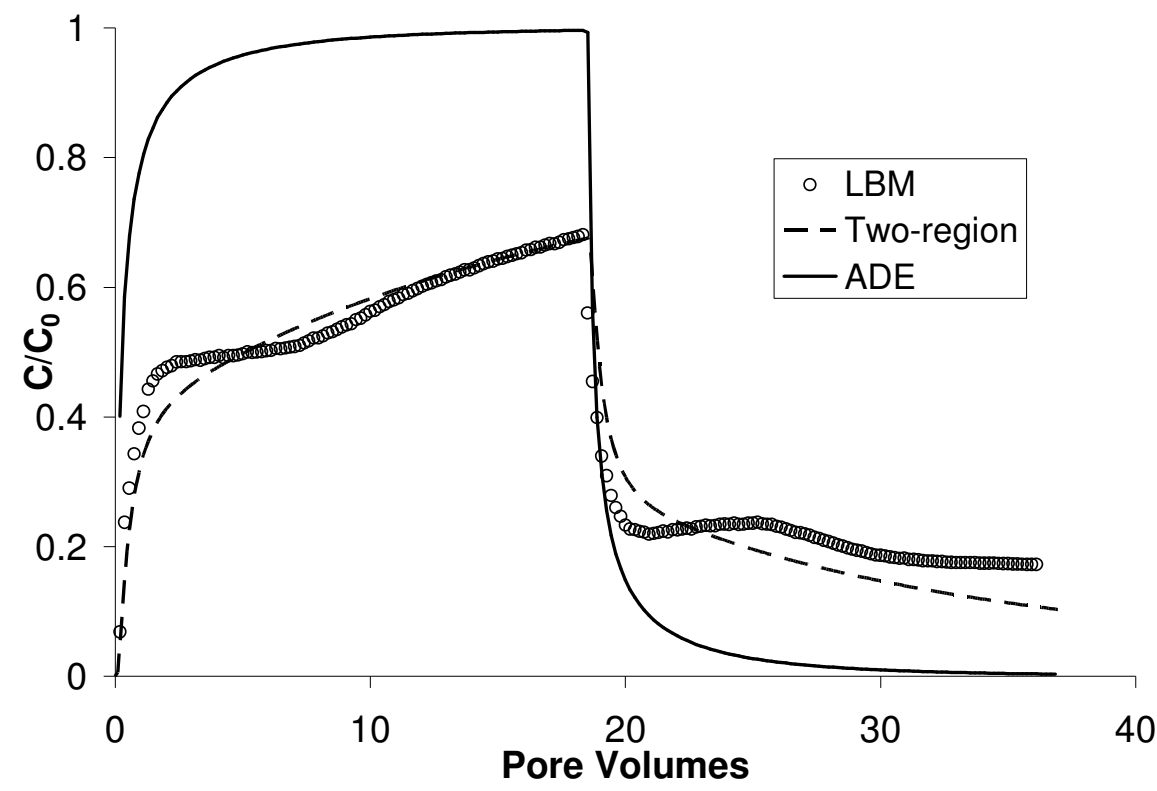

Figure 28. Effluent breakthrough curve simulated with the LBM and fitted two-region and advection-dispersion equation (ADE) for inertial flow conditions $(\operatorname{Re}=900)$ in domain of Figure 27.

In Figure 28 the effluent breakthrough curve is fitted with the advectiondispersion equation and two-region models shown as solid and broken lines respectively. Limitations of the advection-dispersion equation for fitting such results are apparent. The two-region model could more successfully fit the peak and tailing of the breakthrough 
curve, at least for short pulse duration as shown here, which is smaller than required for complete breakthrough from the porous medium; a longer injection period would have lead to a more complex curve like that shown in Figure 24. Conversely, the pulse duration is very long relative to the time scale of transport and eddy mixing in the conduit; these would have prominent influences on the breakthrough only for much shorter pulses. 


\section{TRANSIENT GROUNDWATER FLOW SIMULATION IN CONFINED AQUIFERS}

Investigation of aquifer response to a pumping well is a common hydrological task and forms the basis of techniques for measuring the aquifer parameters, transmissivity $(T)$ and storativity $(S)$. In the simplest case, the well is assumed to penetrate through the full thickness of a homogeneous and isotropic aquifer and flow to the well is horizontal, so that equipotential (equal head) surfaces are vertical. Pumping a well causes a decrease in head/potential in the neighboring region as shown in Figure 35 and develops cylindrical equipotential surfaces concentric on the well. Flow lines are radially inwards towards the pumping well. Head measurements from observation wells at different radii from the pumping well will be unique (Bear, 1972). This is naturally a cylindrical coordinate problem, but numerically it is solved in the Cartesian coordinate system.

In the groundwater flow equation, an injection (source) or pumping (sink) well can be considered as a point source/sink and be represented by a node. The change in head $(s)$, at any distance $r$, from the pumping well, compared to the original water level is drawdown and drawdown plotted against time is a drawdown curve. Any change in the head causes changes in storage for both confined and unconfined aquifers. The discharge, $Q$ coming from the well must be equal to the aquifer yield, which is equal to product of storage coefficient $S$ and rate at which head declines, integrated over the effective surface area of aquifer. 
The storativity or storage coefficient $(S)$ has a strong influence on the drawdown curve. It indicates a combined effect of the elasticity of the rock and the compressibility of the water. In the LB model, the storage coefficient is equal to the relaxation time $\tau$, hence $S$ should always be greater than 0.5 . This does not correspond to storage coefficients observed in the real world, which are typically $10^{-1}$ to $10^{-7}$ for confined aquifers. Thus, a non-dimensional number $\xi$ (as explained below) must always be used to scale hydraulic parameters and time.

Transient change in ground water potential, as a response to localized change in head due to pumping or recharge is an interesting problem in groundwater reservoir management. The transmissivity of an aquifer represents the volumetric flow rate $Q$ per unit width of aquifer $W$ under a unit hydraulic gradient $\nabla h$. Volumetric flux is computed using the transmissivity ( $T=K b$ ) of aquifer of saturated thickness $b$ and hydraulic conductivity $K$, as

$$
Q=T W \nabla h
$$

Ground water head distribution follows the transient ground water flow equation as shown below

$$
\frac{\partial h}{\partial t}=\frac{T}{S} \nabla^{2} h
$$

where $h$ is the head (hydraulic potential) of water at any time $t$ in an aquifer of transmissivity $T$ and storage coefficient $S$. The storage coefficient, $S$ indicates the volume of yield per unit change in head per unit surface area of aquifer. This is a nondimensional parameter. Since LB simulations are not conducted in physical units we need to use non-dimensional numbers to enforce similarity between simulations and physical 
problems. Because the transient ground water equation as shown in equation (59) is analogous to the diffusion equation, we can use the idea of diffusion length to relate physical time with lattice time steps ( $t s)$.

Diffusion length, $L_{D}$ is defined as the distance traveled by means of diffusion alone for time $t$ by a particle in solution with diffusion coefficient $D_{e}$

$$
\xi=\frac{D_{e} t}{L_{D}{ }^{2}},
$$

where $\xi$ is a non-dimensional number. For ground water problems, $D_{e}$ is the hydraulic diffusivity $(T / S)$.

Darcy's law is applicable only for the Stokes flow regime on the representative elementary volume (REV) scale and above, and gives an averaged macroscopic flux for a porous medium. Following application of the conservation of mass (or volume for incompressible flow) to a porous medium above the REV, Darcy's law is the constitutive equation used to derive the transient groundwater flow equation (59), which is completely analogous to the well-known heat equation (e.g., Narasimhan (1999)) and can be written with (equation (59)) and without (equation (61)) a source/sink term.

\subsection{Transient groundwater flow equations}

In the absence of source/sink terms, groundwater head distribution follows the transient ground water flow equation as shown above in equation (59). The transient groundwater flow equation for a domain with a pumping well (i.e., a source or sink) in a confined aquifer is (Wang and Anderson, 1982): 


$$
\frac{\partial h}{\partial t}=\frac{T}{S} \nabla^{2} h+\frac{q}{S}
$$

These two equations have very important differences. In equation (59), only the ratio of hydraulic diffusivity $(T / S)$ is important and the two hydraulic parameters can not be separated. The presence of a source/sink term in equation (61) allows distinct contributions from the hydraulic parameters, $S$ and $T$.

In equations (59) and (61), the coefficient on the gradient term on the RHS represents the hydraulic diffusivity of the aquifer, analogous to the coefficient of molecular diffusion in the diffusion equation and the thermal diffusivity in the heat equation. As noted by Ginzburg (2005), the LB-based diffusion model represents another strategy for solving the transient ground water flow equation. Recently Camas Serván (2007) solved the Henry problem (Henry, 1964) using an LB-based diffusion model. The diffusion coefficient controls the mixing of tracer with background fluid. Similarly, hydraulic diffusivity $(D=T / S)$ for an aquifer controls the change in head across the aquifer over time. The analogy between the diffusion equation and the transient flow equation can be used to solve head distribution over the aquifer using the lattice Boltzmann model for the diffusion problem by setting $u_{A}=0$ in equation (10) and equating the head with the density of the solute component, as head and density both are scalar properties.

\subsection{Boundary value problem in a confined aquifer without source/sink}

Wang and Anderson (1982) present a transient groundwater flow problem in which an aquifer responds to a sudden change in one of the boundary conditions. The 
aquifer is assumed one-dimensional and is confined by impermeable layers on top and bottom as shown in Figure 29. In this case, there is no source/sink and the transient ground water flow equation (59) 1-D becomes in

$$
\frac{\partial h}{\partial t}=\frac{T}{S} \frac{\partial^{2} h}{\partial x^{2}}
$$

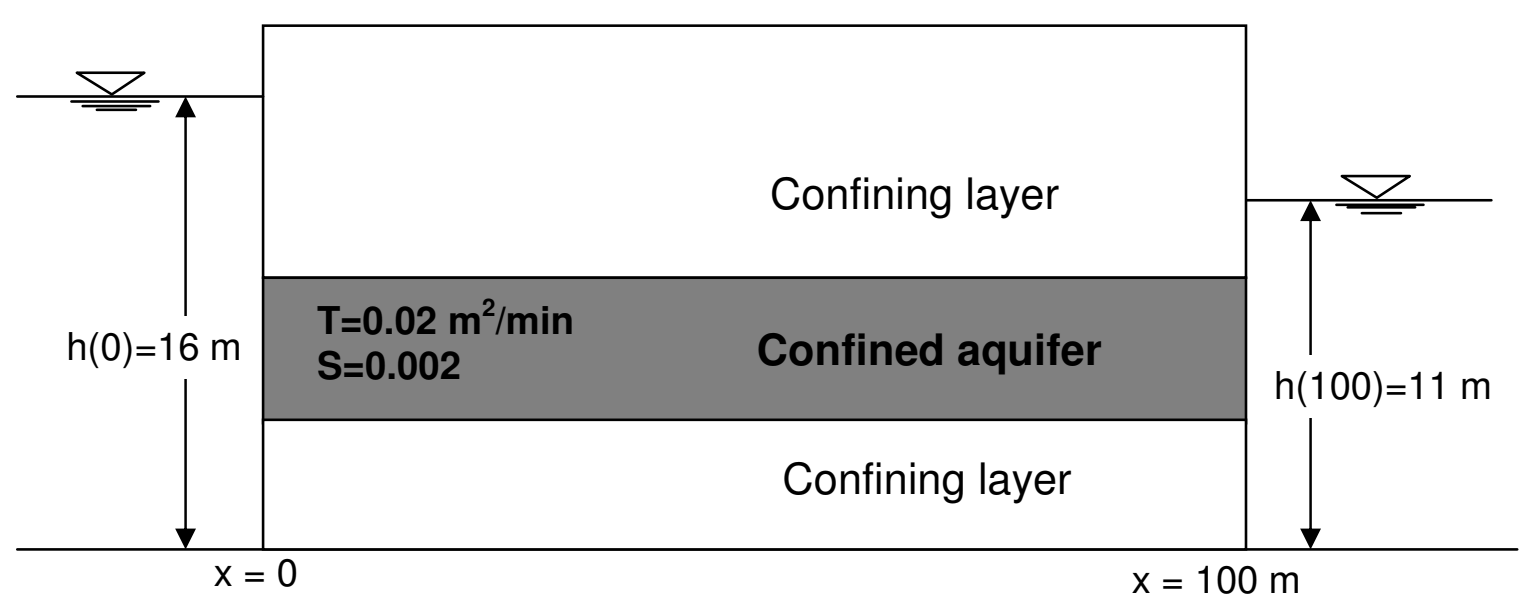

Figure 29. Schematic representation of confined aquifer with boundary conditions.

The aquifer is connected by reservoirs at $x=0$ and $x=100 \mathrm{~m}$. Initially the aquifer has uniform head equal to $16 \mathrm{~m}$. The aquifer parameters are $T=0.02 \mathrm{~m}^{2} / \mathrm{min}$ and $S=$ 0.002; at $t=0+$ the reservoir boundary on the right instantly falls to $h=11 \mathrm{~m}$. The aquifer responds to this sudden change in head by ultimately reaching a steady-state, linear head distribution between the reservoirs. Since the transient ground water equation in equation (59) and (62) is analogous to the diffusion equation, the idea of diffusion length as shown in equation (60) can be used to relate the physical time with the lattice time step (ts). $\xi$ is a non-dimensional number, which will be identical in physical and lattice units. 


\subsection{LB-based diffusion model}

First the transient reservoir problem shown in Figure 29 is solved with the LBbased diffusion model. LB and physical parameters are related using a non-dimensional number $\xi$. To achieve the same $\xi$ value, we use the LB model of domain size $100 l u \times 10$ $l u$ and the model relaxation time parameter is $\tau_{B}=0.8 \mathrm{ts}$, which leads to $\mathrm{D}=0.1 \mathrm{lu^{2 }} / \mathrm{ts}$ according to equation (11). The diffusivity in the LB model is reduced by 100 times and the time step is increased by 100 times compared to the physical values to keep $\xi$ the same as in the physical problem at each simulated time (Table 3).

\begin{tabular}{|l|l|l|l|l|l|l|l|l|l|}
\hline $\mathrm{T}_{\text {physical }}$ & $\mathrm{S}_{\text {physical }}$ & $\mathrm{D}_{\text {physical }}$ & $\mathrm{t}_{\text {physical }}$ & $\mathrm{L}_{\text {Dphysical }}$ & $\xi$ & $\mathrm{L}_{\text {DLBM }}$ & $\mathrm{D}_{\text {LBM }}$ & $\tau_{\mathrm{B}}$ & $\mathrm{t}_{\text {LBM }}$ \\
\hline $\mathrm{m}^{2} / \min$ & & $M^{2} / \min$ & $\min$ & $m$ & & $l u$ & $l u^{2} / t s$ & & $t s$ \\
\hline 0.02 & 0.002 & 10 & 10 & 100 & 0.01 & 100 & 0.1 & 0.8 & 1000 \\
\hline 0.02 & 0.002 & 10 & 100 & 100 & 0.1 & 100 & 0.1 & 0.8 & 10000 \\
\hline 0.02 & 0.002 & 10 & 400 & 100 & 0.4 & 100 & 0.1 & 0.8 & 40000 \\
\hline
\end{tabular}

Table 3. Parameters in physical and lattice units as used in LB-based diffusion model for reservoir problem of Figure 29.

The results are plotted in Figure 30 for $1000 \mathrm{ts}(10 \mathrm{~min}), 10000 \mathrm{ts}(100 \mathrm{~min})$ and $40000 \mathrm{ts}$ (400 $\mathrm{min}$ ). This demonstrates that the LB-based diffusion model can solve the transient ground water head distribution.

Without a source/sink, the only parameter we can change in this model is hydraulic diffusivity via the relaxation time $\tau_{B}$. Thus, it is not possible to simulate groundwater problems governed by equation (61) and characterized by the potential for 
independent variation of $S$ and $T$ with this model in its current form; source/sink terms would have to be added to the LB model.

\subsection{Altered-velocity flow model}

It is also possible to solve transient groundwater flow problems using the LBbased altered-velocity flow model described in section 3.3.2. We simulate the same boundary value problem as shown above in Figure 29 using the altered-velocity flow model here. First we need to resolve the relationships between the hydraulic parameters $T$ and $S$ and the LB parameters. Transmissivity depends on the hydraulic conductivity, which is a function of permeability and fluid viscosity. Permeability is linked with the LB parameter $\mathbf{R}$ and the kinematic viscosity following equation (18); hence transmissivity (T) will be associated with $\mathbf{R}$, and dependence on $\tau$, which controls kinematic viscosity, is also expected. The storage coefficient is a hydraulic parameter that controls the transient behavior of a confined aquifer, and $\tau$ is an LB parameter that controls the time evolution of particle dynamics. Hence, $S$ is expected to be closely linked with $\tau$. Based on testing of LB simulations against the analytical Theis solution described below for different values of $\mathbf{R}$ and $\tau$, transmissivity $T$ is associated with resistance field $\mathbf{R}$ as

$$
T=\frac{\tau \mathbf{R}^{-1}}{3}
$$

and the storage coefficient $S$ was observed to be equal to the relaxation time $\tau$.

The parameters used in the altered-velocity model of the transient ground water flow problem of Figure 29 without source/sink are shown in Table 4. 


\begin{tabular}{|l|l|l|l|l|l|l|l|l|}
\hline$t_{\text {physical }}$ & $\tau$ & $\mathbf{R}$ & $T_{L B M}$ & $S_{L B M}$ & $D_{L B M}$ & $\xi$ & $L_{D}$ & $t_{L B M}$ \\
\hline $\min$ & $t s$ & $t s^{-1}$ & $l u^{2} / t s$ & -- & $l u^{2} / t s$ & -- & $l u$ & $t s$ \\
\hline 10 & 0.96 & 1 & 0.32 & 0.96 & $1 / 3$ & 0.01 & 100 & 300 \\
\hline 100 & 0.96 & 1 & 0.32 & 0.96 & $1 / 3$ & 0.1 & 100 & 3000 \\
\hline 400 & 0.96 & 1 & 0.32 & 0.96 & $1 / 3$ & 0.4 & 100 & 12000 \\
\hline
\end{tabular}

Table 4. Parameters used in altered-velocity model.

The following procedure was adopted to obtain agreement between $\xi$ in physical and LBM units. First, resistance and relaxation time parameters were arbitrarily selected as $\mathbf{R}=1 \mathrm{ts}^{-1}$ and $\tau=S=0.96$, and the length scale was fixed to $1 \mathrm{~m}=1 \mathrm{lu}$. The transmissivity $(T)$ of the aquifer was then $0.32 l u^{2} / t s$ and the storage coefficient was equal to 0.96 ; hence, the hydraulic diffusivity $(T / S)$ is $1 / 3 l u^{2} / t s$. Diffusion length as expressed in equation (60) is used to scale time steps between physical units and lattice units as shown in Table 4. 


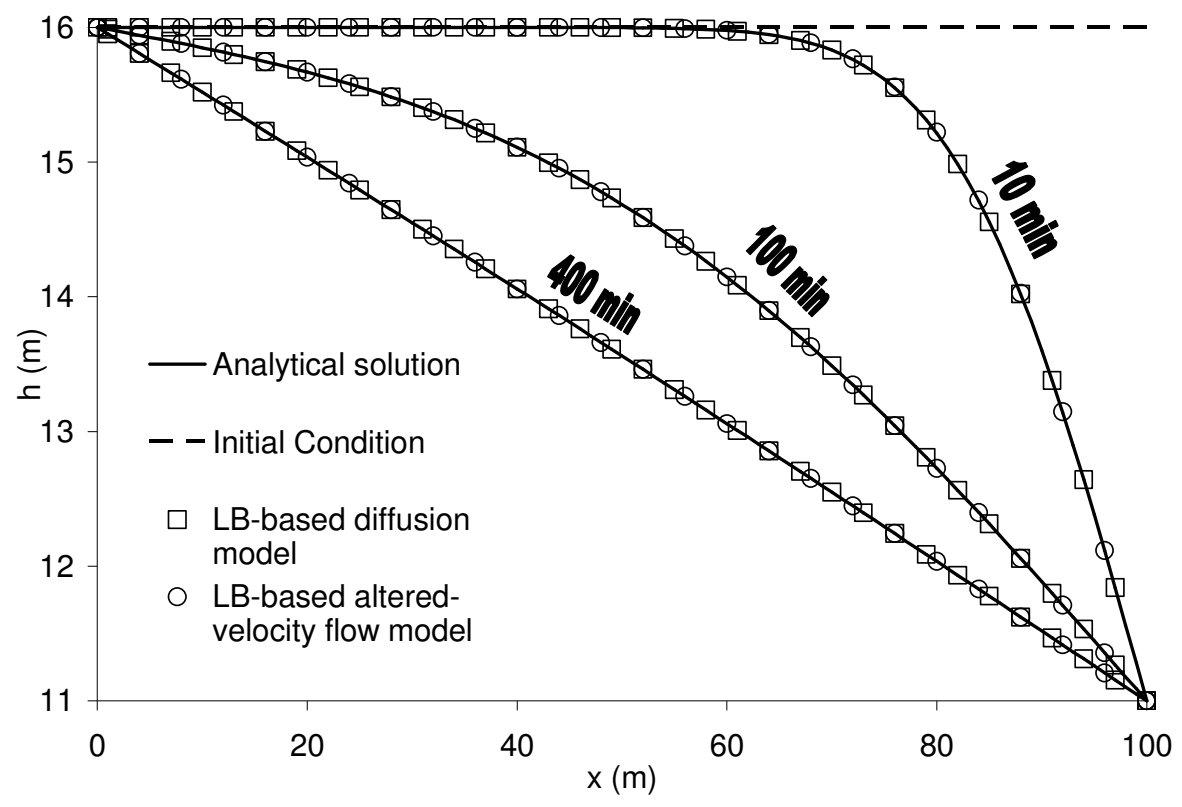

Figure 30. Transient change in head as a response to a sudden change in head at right boundary for two different solutions techniques vs. analytical results.

The LB solution shows an excellent match with the analytical solution (Figure 30). This demonstrates the ability of the LB-based altered-velocity flow model to simulate an initial value problem from the groundwater discipline.

\subsection{Source/Sink in groundwater flow model}

In the LBM, source/sink nodes can be implemented by adding and removing the desired mass flux $(\rho \times q)$ at the source and sink nodes respectively. To assess the accuracy of this approach, one-and two-dimensional simulations are conducted. To demonstrate conservation of mass, the mass flux across any appropriate section for one-and twodimensional cases can be computed. 
A $50 l u \times 10 l u$ domain (Figure 31 ), bounded at left and right boundaries, is used to simulate one-dimensional flow with source and sink nodes. The initial fluid density is uniform $\left(1 m u / l u^{2}\right)$ all over the domain. A line source and sink are set at $3 l u$ from the left and right boundaries respectively. As shown in Figure 31, the black line is the boundary at each end, the dark line near the left boundary indicates the source region and white line near the right boundary indicates the sink region.

A constant discharge of $0.0051 \mathrm{mu} / \mathrm{ts}$ (a small value is chosen to avoid compressibility effect) is set to be added and removed at each node at the line source and sink. The computed flux across any section is found to be $0.0051 \mathrm{mu} / \mathrm{ts}$.
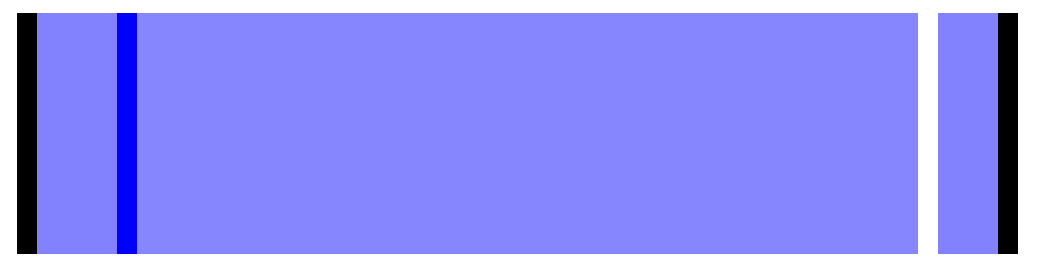

Figure 31. $50 l u \times 10 l u$ domain to simulate 1-D flow with line source and sink nodes. Black lines at two ends represent solid boundaries (wall). The dark blue vertical line near the left end represents line of source nodes (density higher than neighboring nodes) and the white vertical line near the right end represents line of sink (density lower than neighboring nodes). A pressure gradient is created between source and sink due to imposed density difference.

This verifies that conservation of mass is honored in one-dimensional simulation of source and sinks nodes in a homogeneous porous medium using the LBM.

To test the source/sink node in a 2-D flow field, $51 l u \times 51 l u$ domain with source $(26,17)$ and sink node $(26,34)$ is chosen as shown in Figure 31 . The domain has uniform $\mathbf{R}$ equal to $0.1 \mathrm{ts}^{-1}$. 


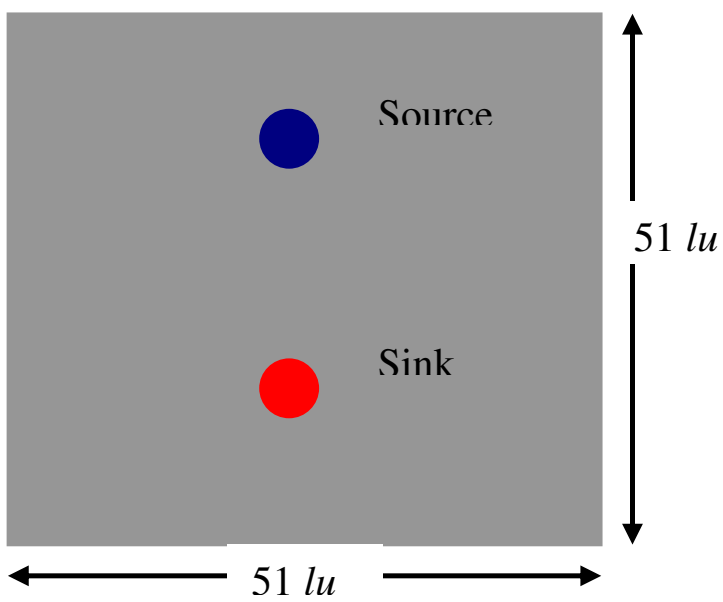

Figure 32.51 $l u \times 51 l u$ domain with source and sink nodes with porous media in background.

The pressure difference between source and sink drives the flow in two dimensions from source to sink. The flow field is solved for two different kinds of boundary conditions; one is no-flow and another is constant pressure on all four boundaries. The initial density over the whole domain is set equal to $1 \mathrm{mu} / \mathrm{lu}^{2}$. 


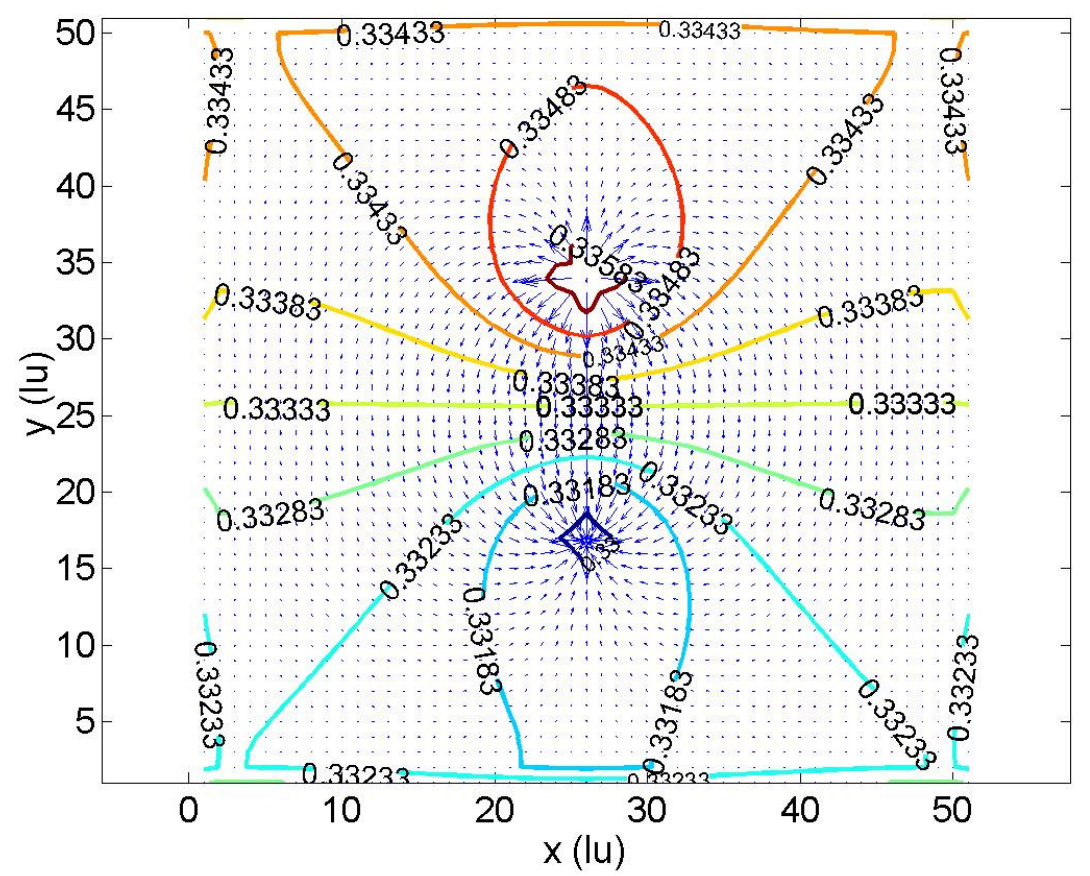

Figure 33. Quiver plot (velocity vector) with pressure contour lines for domain with walls on all four sides.

Since the flow occurs orthogonal to the lines of constant pressure, total flux is equal to the sum of flux measured across all the nodes falling on lines of constant pressure. The flux is measured at a section drawn parallel to the $x$-axis in the middle of domain. The measured flux is found to be equal to $0.068 \mathrm{mu} / \mathrm{ts}$ against the flux equal to $0.068 \mathrm{mu} / \mathrm{ts}$ set at the source/sink nodes.

Figure 34 shows a velocity vectors or flow field (quiver plot) for a 2-D flow field with constant pressure conditions on all four boundaries. Solid lines are pressure contours. Pressure differences between the source and the boundary closer to the source node will cause a flow directed towards the boundary, whereas flow will be from the 
boundary to the sink near the sink node. Hence, the total mass flow is not direct from source to sink; instead only half of the mass is directed from the source towards the sink, due to the symmetric arrangement of the source/sink nodes in the domain.

The flux is measured at a cross-section parallel to $x$-axis in the middle of domain. The measured flux is found to be equal to $0.034 \mathrm{mu} / \mathrm{ts}$ against the flux equal to 0.068 $m u / t s$ set at source/sink nodes. The flux computed along the cross-section is half the flux set at the source/sink.

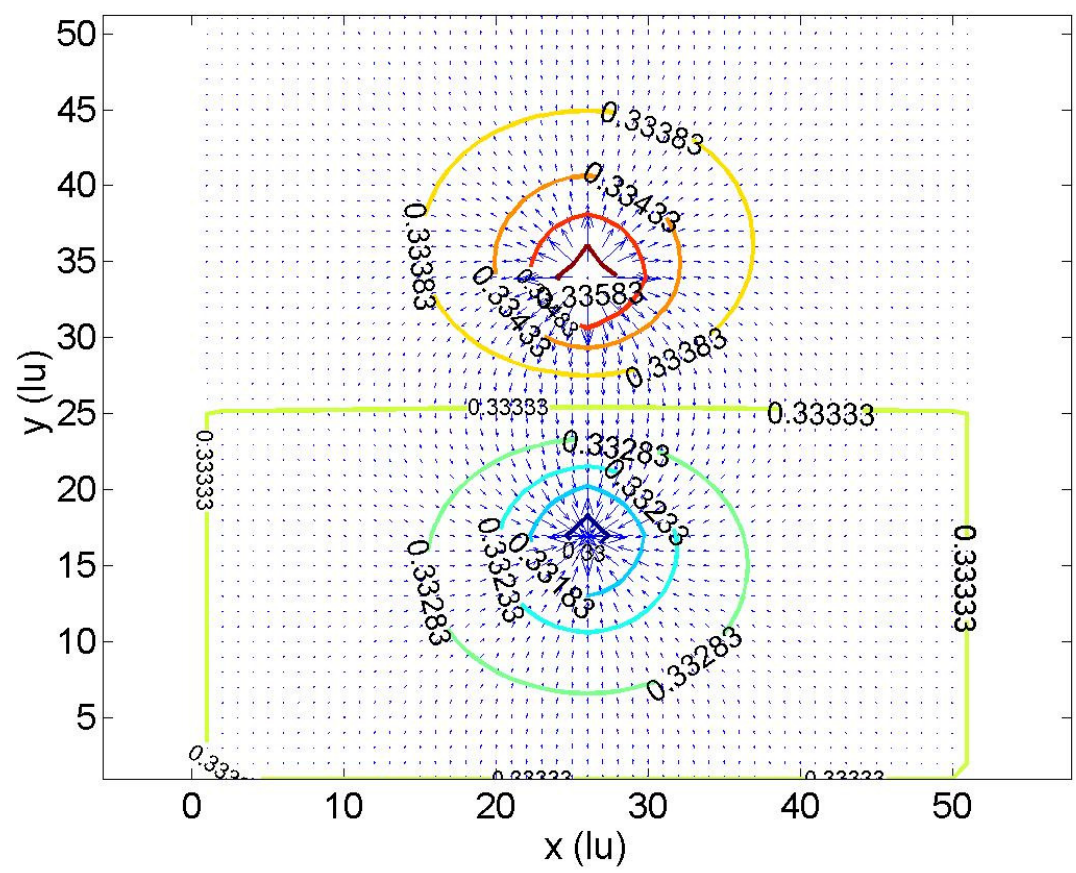

Figure 34. Quiver plot (velocity vector) with pressure contour lines for domain with fixed pressure boundary on all four sides. 


\subsection{Transient ground water flow problem with source/sink}

Due to injection or pumping, there is a change (rise or drop) in head/water-table in the vicinity of the well. This change in head $(s)$, at any distance ' $r$ ' from the pumping well, compared to the original potentiometric surface or water table is called the cone of depression as shown in Figure 35. Any change in head causes changes in storage for both confined and unconfined aquifers. The well is assumed to penetrate through the full thickness of aquifer and flow is horizontal, so that equipotential lines are vertical. Hence, the observation from observation wells at different radii from a pumping or injection well will be unique (Bear, 1972).

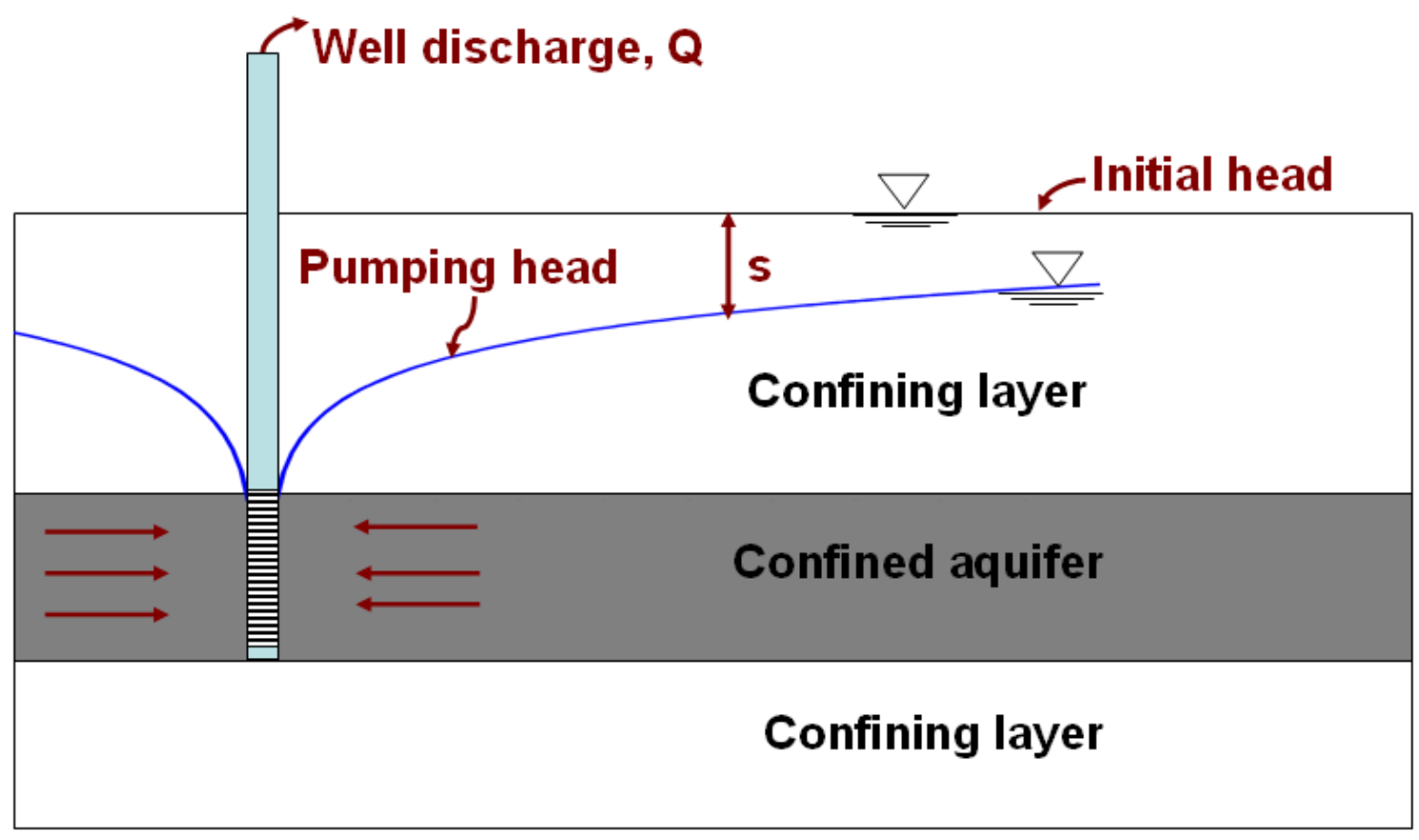

Figure 35. Schematic representation of drawdown in a confined aquifer for a constant pumping rate (Q).

The volumetric rate of flux is fixed at the sink node and the drawdown curve is observed in the neighboring region. The discharge, $Q$ coming from the well must be 
equal to the aquifer yield, which is equal to product of storage coefficient $(S)$ and rate at which head declines, integrated over the effective surface area of aquifer (Osiensky et. al. 2000)

$$
d Q=-S r d \theta d r \frac{\partial h}{\partial t}
$$

The aquifer is assumed homogeneous in angular direction $(\theta)$, hence flow $(Q)$ will depend only on $r$ :

$$
Q(t)=-2 \pi S \int_{r_{w}}^{\infty} r \frac{\partial h(r, t)}{\partial t} d r
$$

The differential form of the transient ground water flow equation in cylindrical coordinates is

$$
\frac{1}{r} \frac{\partial}{\partial r}\left(r \frac{\partial h}{\partial r}\right)=\frac{S}{T} \frac{\partial h}{\partial t}
$$

Theis (1935) derived an analytical solution for equation (66) for the following boundary and initial conditions

$$
\begin{aligned}
& h(r, 0)=h_{0} \\
& h(\infty, t)=h_{0} \\
& \lim _{r \rightarrow 0}\left(r \frac{\partial h}{\partial t}\right)=\frac{Q}{2 \pi T} \quad \text { for } t>0
\end{aligned}
$$

$h_{0}$ is initial head. The Theis solution for drawdown $(s)$ is

$$
s=\frac{Q}{4 \pi T} \int_{u}^{\infty} \frac{e^{-u}}{u} d u
$$

where $u=\frac{r^{2} S}{4 T t}$ and $u$ is a dummy integration variable, which determines the radius of the cone of depression. Transmissivity $T$ controls the overall shape and extent of the cone, and storage coefficient $S$ controls the volume $V$ of the cone of depression in a confined aquifer, which can be written as (Osiensky et. al., 2000) 


$$
V=\frac{Q}{S} t
$$

The drawdown curve is different for confined and unconfined aquifers because in an unconfined aquifer the saturated thickness changes with pumping. The saturated thickness remains constant for confined aquifers. Hence, confined aquifers are simpler to study and model compared to unconfined aquifers.

The confined aquifer is simulated as a $500 l u \times 500 l u$ domain with pressure boundaries on all four sides and a point sink node of strength equal to $0.051 \mathrm{mu} / \mathrm{ts}$ at the geometric center $(250,250)$ of the domain. Initially, there is uniform density of $1 \mathrm{mu} / \mathrm{lu}^{2}$ over the whole domain; this corresponds to an initial drawdown of zero. The drawdown $(s)$ is observed at $r=4 l u$ from the sink node. In drawdown simulations, it is important to observe the effect of boundaries on the transient solution and necessary to consider the propagation of drawdown out to the boundaries (Anderson and Woessner, 1992). In the Theis solution, drawdown is due to pumping alone; the boundaries are at infinite distance and the constant pressure boundary condition in the model should not influence the transient change in head. Hence, the drawdown is observed until the effect of pumping reaches the boundary, which gives the result of an effectively infinite domain.

The resistance tensor $\mathbf{R}$ is set equal to $1 \mathrm{ts}^{-1}$, hence the transmissivity is $1 / 3 \mathrm{lu}^{2} / \mathrm{ts}$ and storage coefficient $(S)$ is to be equal to the relaxation parameter $(\tau=1 t s)$ of the fluid. The simulated drawdown is compared against the Theis solution (Theis, 1935). The result shows an excellent match to the drawdown curve as shown in Figure 36. The open circles 
are LB results of drawdown after every $180 \mathrm{ts}$ and the solid line represents the analytical solution. The fitted storage coefficient is 1 and the transmissivity is $1 / 3 \mathrm{lu}^{2} / \mathrm{ts}$.

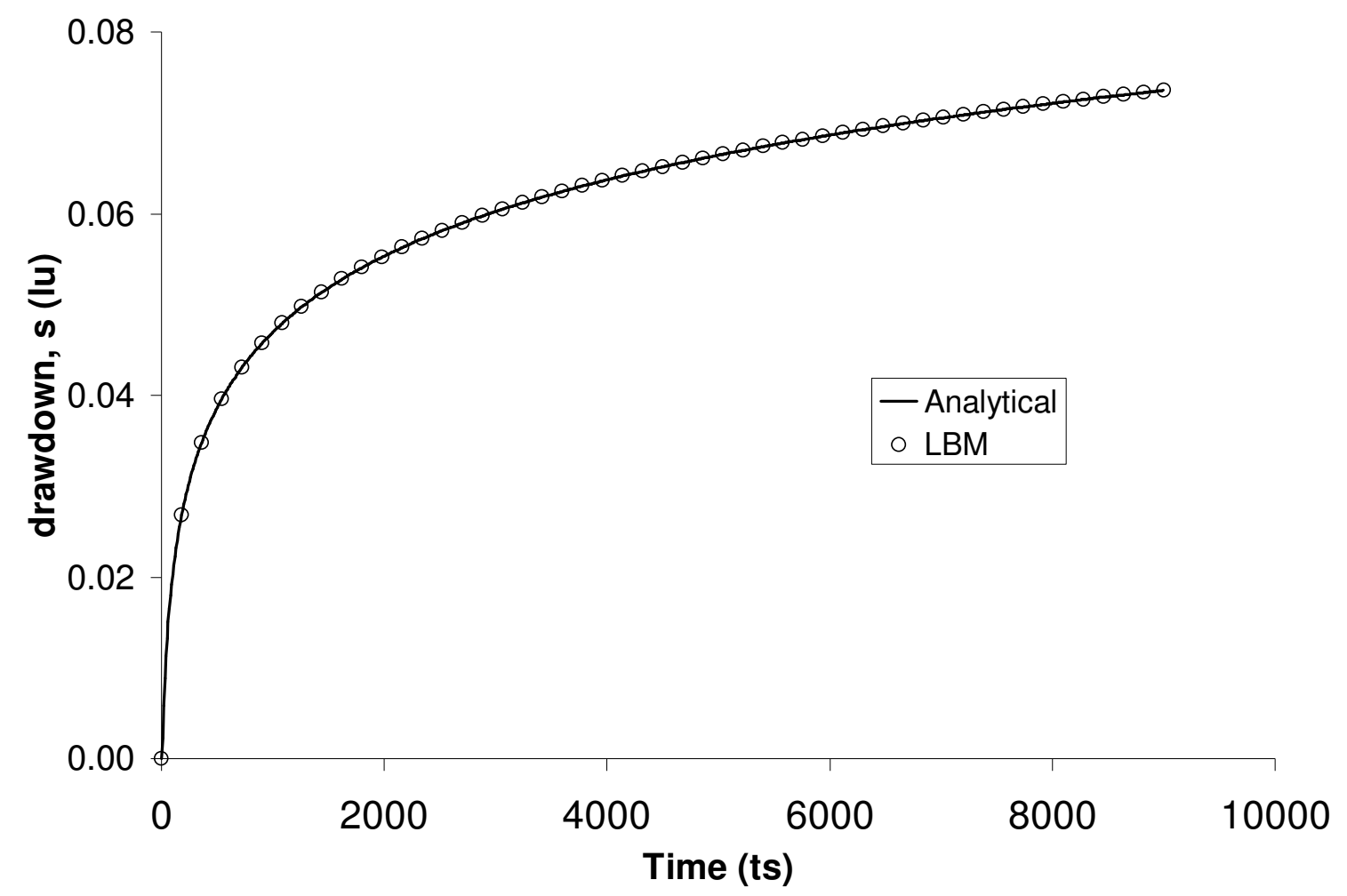

Figure 36. Drawdown curve at observation well in a confined aquifer compared against the Theis solution.

Results for different storage coefficients are shown in Figure 37. The drawdown curve is drawn for a node at $4 l u$ from the pumping well for different values of storage coefficient $(S)$, while keeping the hydraulic diffusivity $(D)$ constant. $\mathbf{R}$ is equal to $1, D$ equals $1 / 3 l u^{2} / t s$, and the pumping rate is equal to $0.051 \mathrm{mu} / \mathrm{ts}$. The LB results are plotted after every $150 \mathrm{ts}$ as shown by open symbols and the analytical solutions are shown by solid lines. There is some departure from the analytical solution as $S$ or $\tau$ gets closer to 0.5. In this model, the hydraulic parameters $T$ and $S$ are treated separately, unlike in the 
LB-based diffusion model without a source/sink, where these parameters are lumped into one as hydraulic diffusivity.

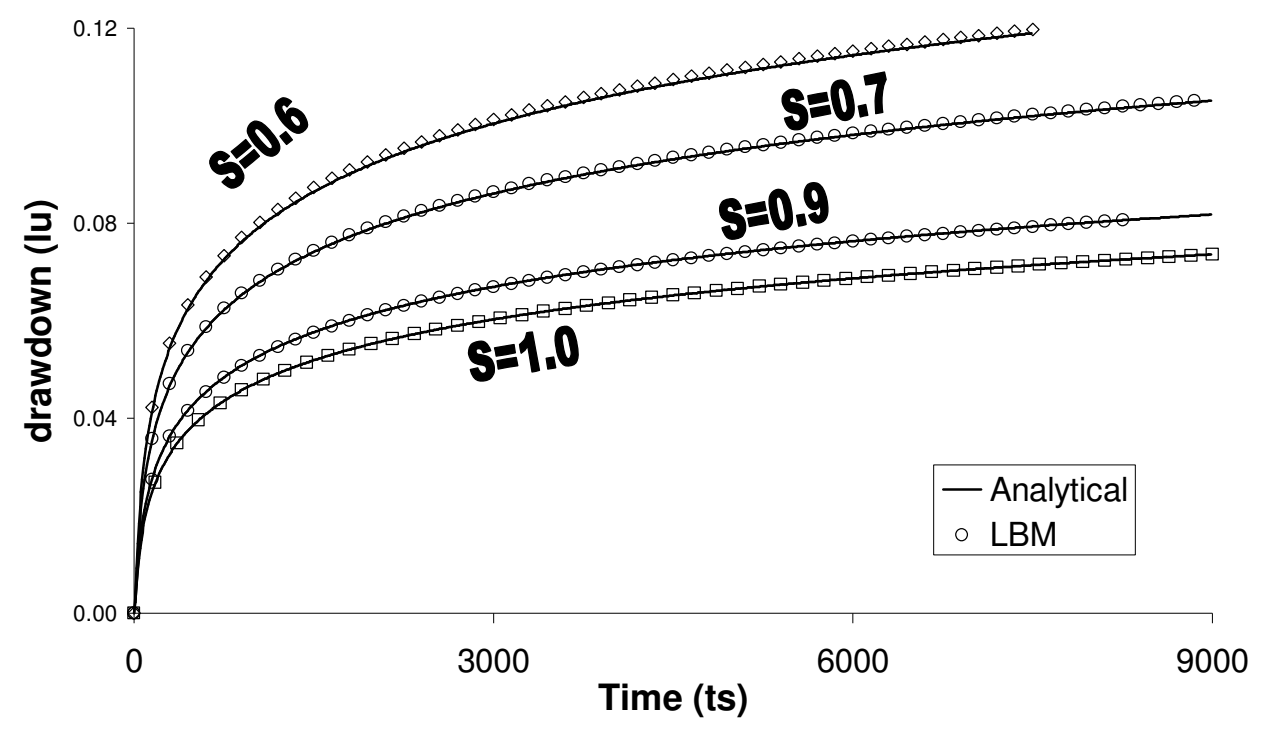

Figure 37. Theis curve for constant hydraulic diffusivity $(D)$ and different storage coefficient $(S)$.

\subsection{Validation of LB model with field data for drawdown data in a confined aquifer}

The altered-velocity LBM for transient ground water flow modeling is verified against field-scale pumping well data (Lohman, 1972) for drawdown $(s)$ in a confined aquifer. These drawdown data are obtained from a well pumping at a constant rate equal to $96,000 \mathrm{ft}^{3} / \mathrm{d}$. The drawdown is measured at $r=400 \mathrm{ft}$ and $200 \mathrm{ft}$ from the pumping well. Hydraulic parameters $T$ and $S$ are found using the curve fitting method. 
The hydraulic diffusivity $D=T / S$ in metric units is equal to $6.84 \times 10^{6} \mathrm{~m}^{2} / \mathrm{d}$. $L$ is a characteristic length chosen equal to $r=400 \mathrm{ft}$ or $122 \mathrm{~m}$. In the LBM, $\tau=1$ t $t$ and $\mathbf{R}=$ $1 / 3 t s^{-1}$; hence $T=1 l u^{2} / t s$ and $S=1$; consequently, hydraulic diffusivity, $D=1$. To scale discharge rate, a non-dimensional parameter is used as follows: $Q / T L=(2929.688$ $\left.m^{3} / d\right) /\left(1367.188 m^{2} / d \times 120 m\right)=0.0178$, which must be the same in the physical and LBM simulations. Assuming, $1 l u=30.5 \mathrm{~m}$; hence $r_{L B M}=4 l u$. Then $Q_{L B M}=$ $(Q / T L)\left(L_{L B M}\right) T_{L B M}=0.01784 \times 4=0.07136 l u^{2} / t s$. So, a sink node is set in the center of domain having a pumping rate equal to $0.07136 \mathrm{mu} / \mathrm{ts}$. The drawdown in $l u$ can be converted into SI units using $1 \mathrm{lu}=30.5 \mathrm{~m}$. Figure 38 shows a comparison between LB simulations and field data for drawdown curves measured at $122 \mathrm{~m}$ and $244 \mathrm{~m}$ from the pumping well. It shows a good match between the field and model data.

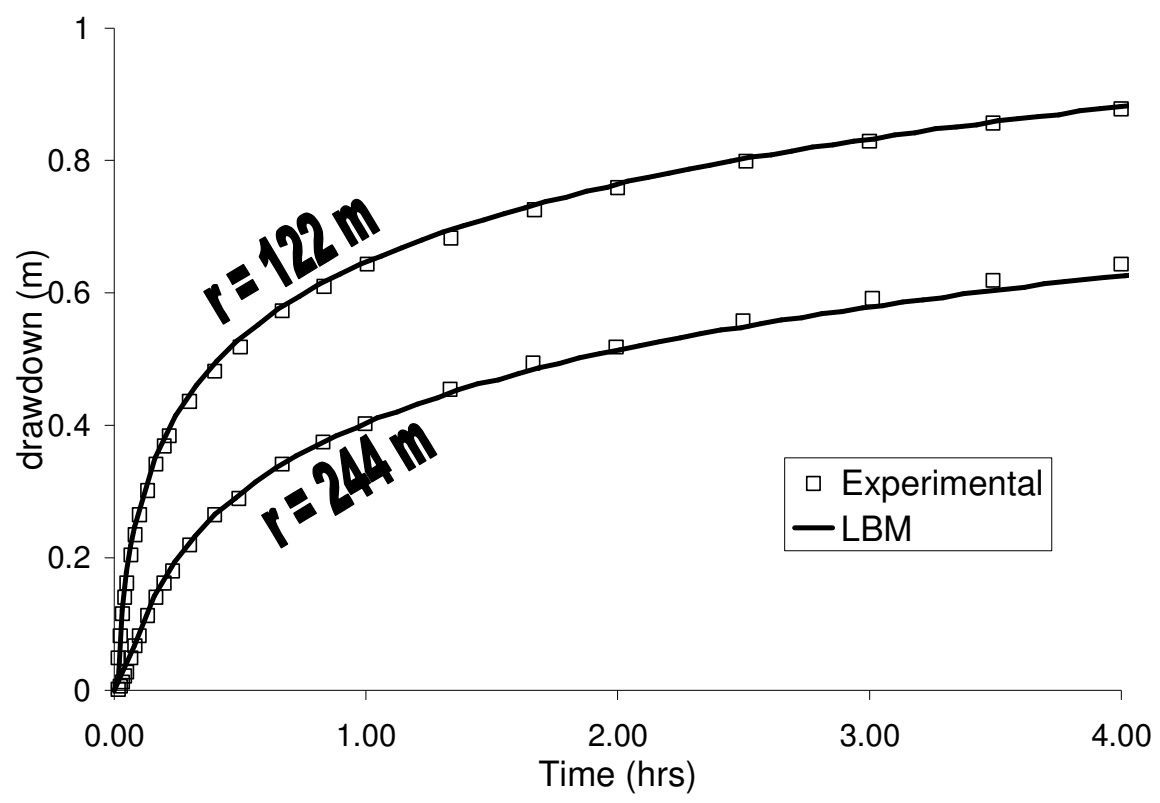

Figure 38. Validation of LB model against the field data for drawdown curve. 
This validation of LB models against field data shows the potential for application of this method for more complicated flow in heterogeneous media such as karst aquifers. Field data for the recovery curve after a pumping test in Miami (well G-3839) and West Palm Beach (well PB-1545) were collected by Reese and Wacker (2007). Wells G-3839 and PB-1545 are open to the Biscayne aquifer. The drawdown curves for the two pumping wells, PB-1545 and G-3839, are shown in Figure 39. The inertial effect on the drawdown curve is apparent in these two curves in the form of water level overshoots and oscillations.

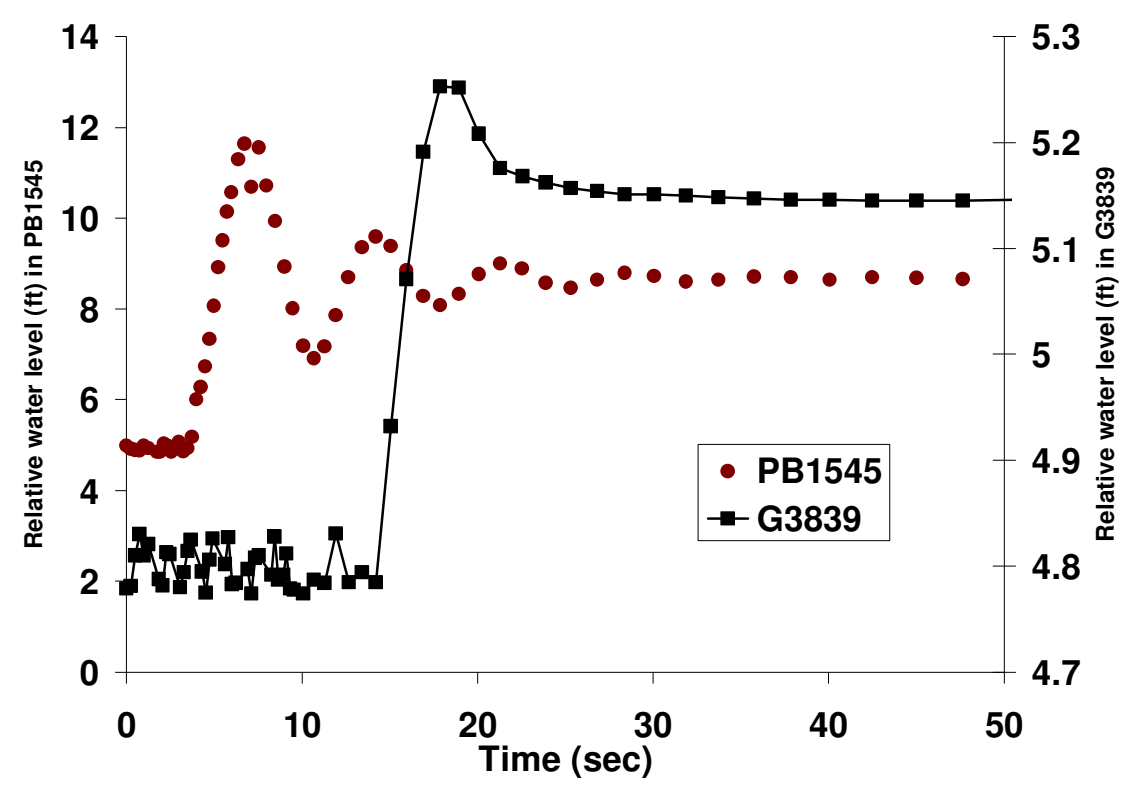

Figure 39. Field recovery data for an aquifer test in Biscayne aquifer. Well PB 1545 is located in West Palm Beach and well G3839 is located in Miami (Reese and Wacker, 2007).

This and similar datasets are suitable for testing for LB models, the simulation of inertial effects during recovery in a karst aquifer. A qualitative comparison for such oscillating drawdown is made using altered-velocity lattice Boltzmann method as follows. 


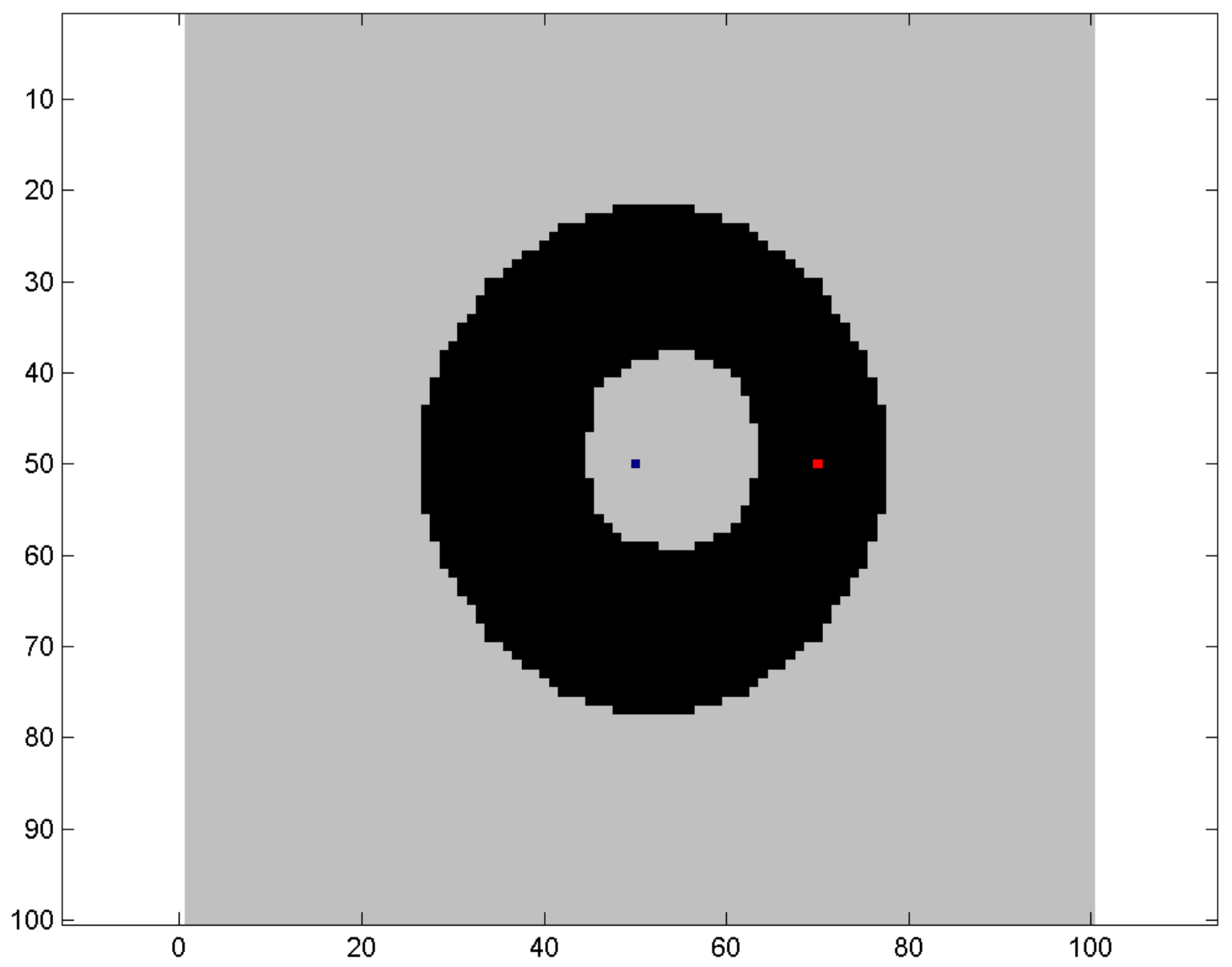

Figure 40. Prototype karst aquifer with circular sinkhole (black) surrounded by porous media (grey). Pumping well is shown in blue and observation well is shown in red.

Figure 40 shows a prototype karst aquifer $(100 l u \times 100 l u)$ with a circular sinkhole (black) and surrounding homogeneous porous media (grey). The pumping well is represented by a blue node $(50,50)$ and observation well is represented by a red node $(70,50)$. Initially, the aquifer is set at uniform head equal to $1 l u$. The pumping well is simulated by enforcing a sink node at the centre of domain having a fixed pumping rate equal to $0.051 \mathrm{lu}^{2} / t s$. Boundaries are periodic and the kinematic viscosity of fluid is $0.1666 l u^{2} / t s$. The simulated drawdown is measured at $20 l u$ from the pumping well. 


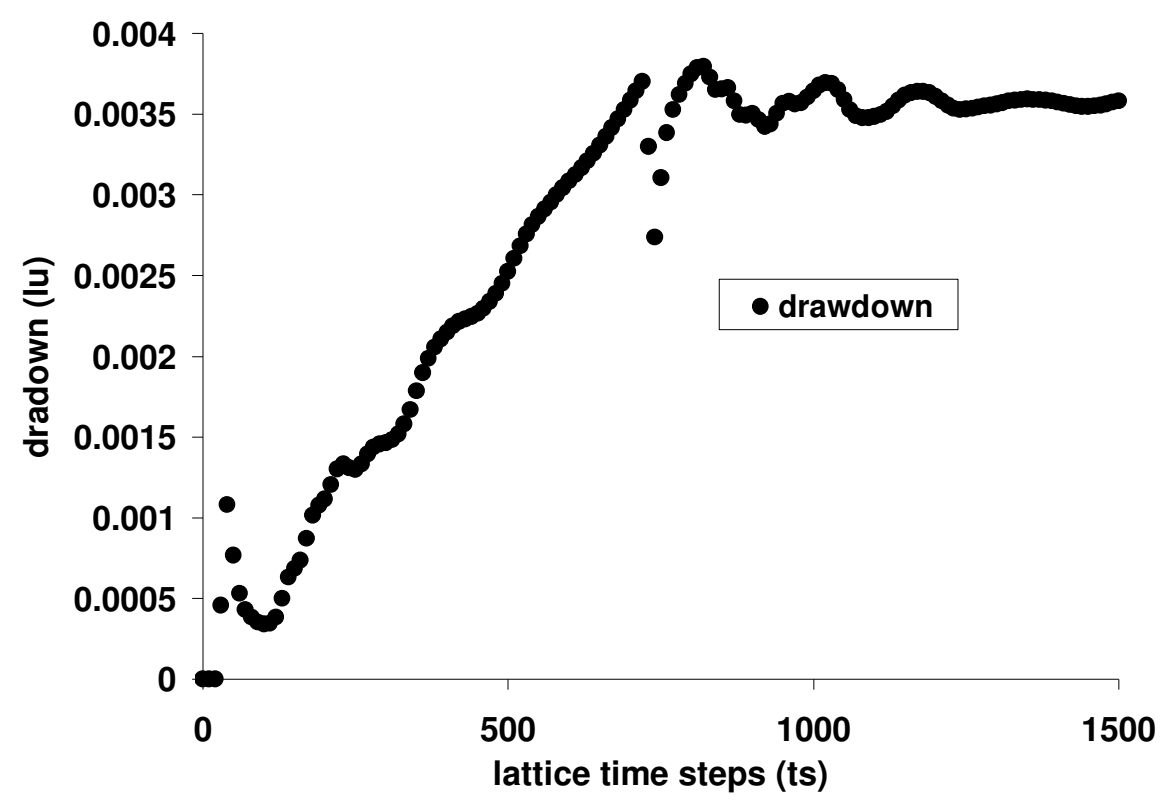

Figure 41. Simulated time-series drawdown in prototype karst aquifer (Figure 40) using alteredvelocity LBM.

The drawdown at observation well is measured by subtracting initial head $(1 l u)$ from head at any time step. The simulated drawdown in the prototype karst aquifer (as shown in Figure 40) is shown in Figure 41. The drawdown exhibits oscillation as observed in karst aquifers in Florida. The prototype karst aquifer is pumped for $700 t s$ at a constant rate and then pumping is stopped. The inertial effect of flow in the karst aquifer causes oscillation in drawdown that dies down to a stable drawdown after $1500 \mathrm{ts}$ after pumping is stopped.

\section{SUMMARY AND CONCLUSIONS}

Solute transport is a complex process in karst aquifers and depends on geological and hydrological characteristic of the domain. Various analytical and numerical models are available for prediction of solute transport. The advection-dispersion equation is the 
most commonly used model for the prediction of breakthrough in porous media and it has been found to have strong dependence on the inlet boundary and geometrical conditions. Four different analytical solutions are available for first- and third-type inlet boundary conditions in finite and semi-infinite domains, which show the effect of inlet boundary conditions on the effluent breakthrough curves. The Peclet number is a dimensionless number widely used to characterize solute transport problems and develop a similitude between two different systems of units. For smaller Peclet number $(P e<20)$ breakthrough curves are found to be different for similar transport parameters due to different inlet boundary conditions or type of domain. The LBM is applied to simulate these analytical solutions in one-and two-dimensions. Resident $\left(C_{r}\right)$ and flux concentrations $\left(C_{f}\right)$ are computed at the effluent section for finite and semi-infinite domains. The LBM simulations match accurately with the analytical solutions.

The LBM is further applied to solve a zero-diffusion problem $(P e \rightarrow \infty)$ under Poiseuille flow, which is another extreme of possible solute transport regimes that could be encountered in karst aquifers. The LBM showed a good match with the analytical solution for zero-diffusion transport in a slit together with the low $P e$ results this verifies the ability of the LBM to simulate boundary value problems in solute transport for the entire range of Peclet numbers.

The advection-dispersion equation has been modified to fit "anomalous transport" observed due to heterogeneity, dead-end pores, fractured porous media or porous media cut by conduits as characteristic of karst aquifers. The two-region model was developed 
from the advection-dispersion equation and is commonly applied to predict long-tailing and the sudden rise in breakthrough curves observed in many situations. A sink term is added in the advection-dispersion equation to link the mobile and immobile regions in porous media and solute in the mobile and immobile regions is linked by an exchange coefficient. This model is very restrictive in approach and limited in mimicking the actual physical process. The LBM is applied in a constrained way to satisfy the inherent assumptions in two-region model and successfully simulate breakthrough curves for two different fractional mobile regions $(\beta)$. The fitted value of flux is close to the expected value and the coefficient of mass exchange showed low correlation with the diffusion coefficient, indicating independence of these parameters. Transport simulation in a more realistic conduit/matrix geometry gave a breakthrough curve with the classic features of rapid initial rise, plateau, and gradual rise towards the maximum concentration. In this circumstance, the two-region model provided an incrementally better fit than the advection-dispersion model, but the limitations of these models for such complex curves were apparent and similar limitations are expected in karst aquifers.

A LBM-based heterogeneous porous media flow model was linked with a LBM anisotropic dispersion solver to simulate solute transport in large-scale heterogeneous porous media. This is necessary for regional-scale karst aquifer simulation. LBM simulation of solute transport in a non-axis-aligned flow field in a homogeneous domain fit the analytical solution of the two-dimensional advection-dispersion equation, building confidence in the model. The same model simulated solute transport in a heterogeneous porous medium. The effluent breakthrough curve could be fitted with the one- 
dimensional advection-dispersion model. Finally, breakthrough from a karst-like system consisting of a conduit with inertial regime flow in a heterogeneous aquifer is compared with the advection-dispersion model and the two-region model. Flow in the conduit region had a high Reynolds number $(R e=900)$, causing eddy mixing of the solute. The two-region analytical solution fit the effluent breakthrough curve reasonably well, but the curve could not be fitted with the advection-dispersion model. The relative success of the two-region model in this case is attributed to the time scale being smaller than required for complete breakthrough from the porous medium; a longer injection period would have lead to a more complex curve. Conversely, short pulse durations relative to the time scale for transport through the porous medium make transport and eddy mixing in the conduit the dominant influence on breakthrough.

Two different kinds of LB-based models are used to solve transient groundwater flow problems. Application of the LBM to this problem is new and critical to a complete, non-steady karst hydraulics model. The analogy between the diffusion coefficient in the diffusion equation and hydraulic diffusivity in the groundwater flow equation is used to implement an LB-based diffusion model to solve the transient groundwater flow equation. The LB-based diffusion model, as currently configured without source/sink capability is found to have limited applicability due to the inherent lumping of two hydraulic parameters ( $T$ and $S$ ) into one parameter hydraulic diffusivity $(D)$. The LBbased altered-velocity flow model is more flexible and able to solve transient problems with or without source/sink terms in its current form. The hydraulic parameters are treated separately in this model. $T$ is linked with the resistance field $(\mathbf{R})$ and relaxation 
parameter $\tau$. The storage coefficient is found to be equal to $\tau$. A non-dimensional number

$(\xi)$ is used to scale time and hydraulic diffusivity between LB units and physical units.

The results from LB-based models showed good agreement with available analytical solutions.

\section{FUTURE WORK}

Many interesting possibilities are open to explore using the LBM because it is a comparatively new scheme and has not been widely applied to environmental hydraulics. In brief, the following are some major topics that deserve further effort:

1. Implementation of a robust multi-relaxation time (MRT)-based LB model to simulate high Peclet number and high Reynolds number systems of flow and transport in karst aquifers.

2. Test higher anisotropy ratios for permeability and dispersivity in LB models.

3. Include a reaction model linked with the flow model to simulate dissolution of karst aquifers.

4. Test LB aquifer hydraulics model to simulate and ultimately fit aquifer and slug tests conducted in the inertial flow regime in karstic aquifers.

This research is believed to be the first attempt to establish the LBM as an alternative model to simultaneously solve fluid flow and solute transport in karst aquifers. Three LB methods are linked to solve inertial flow and consequent eddy mixing in karst aquifers. The LB model results compare well to the analytical solution for the zerodiffusion problem in the laminar flow regime. When the low-diffusion problem is applied 
to high Reynolds number flow however, error in transport estimation increases. This indicates a need for the development of better transport models for high flow/small diffusion problems. There are probably more robust LB methods available (Ginzburg, 2005), which are better capable of solving low-diffusion transport at high flow rates. Application of such models will open a much broader range of flow and transport regimes as required in karst aquifers.

The anisotropy ratio for hydraulic conductivity or dispersivity is 10 or 100 in real physical situations. The LB model applied in this research is not stable for higher dispersivity coefficient anisotropy ratios. Ginzburg (2005) and Camas Serván (2007) have proposed LB models for higher dispersivity anisotropy ratios. Also, rigorous testing is required to explore the stability limits of the macroscopic flow model for different ratios of anisotropic hydraulic conductivity.

Since carbonate aquifers undergo dissolution during flow and the rate of dissolution also depends upon the rate of flow, a reaction model should be linked with the existing flow and transport LB model to simulate the growth of karst under different hydraulic conditions.

The models proposed for solving hydraulic potential in karst aquifers require testing for drawdown estimation in aquifers with high likelihood of significant inertial flow. 


\section{LIST OF REFERENCES}

Alvarez, P.F. 2007. Lattice Boltzmann modeling of fluid flow to determine the permeability of a karst aquifer. M.S. Thesis, Florida International University. pp. 96

Anderson, M. P. and W.W.Woessner. 1992. Applied ground water modeling: simulation of flow and advective transport. Academic Press. pp. 237.

Anwar, S., A. Cortis., and M. C. Sukop. 2008. Lattice Boltzmann simulation of solute transport in heterogeneous porous media with conduits to estimate macroscopic continuous time random walk model parameters. Vol. 8 (1-4): 213 - 221. DOI: 10.1504/PCFD.2008.018092

Anwar, S. and M. C. Sukop. 2008. Regional scale transient groundwater flow modeling using Lattice Boltzmann methods. Progress in Computational Fluid Dynamics. (in press)

Atkinson, T.C., D.I. Smith, J.J. Lavis, and R.J. Whitaker. 1973. Experiments in tracing underground waters in limestones. Journal of Hydrology Vol. 19 (4): 323-349.

Aris. R. 1956. On the dispersion of a solute in a fluid flowing through a tube. Proc. Roy. Soc. Vol. 235A:67-77.

Bai, M., Z. Shu., J. Cao., M. Zaman., J. C. Rogiers. 1999. A semi-analytical solution for a two-dimensional capacitance model in solute transport. Jour. Petro. Sci Engg. Vol. 22: 272-295.

Barenblatt, G. I., I. P. Zheltov., and I. N. Kochina. 1960. Basic Concepts in the theory of seepage of homogeneous liquids in fissured rocks (strata) Journal of Applied Mathematics and Mechanics. Vol. 24 (5): 1286-1303.

Baudet, C., J.P. Haulin, P.Lallemand., and D. d'Humieres. 1989. Lttice-gas automata: A model for the simulation of dispersion phenomena. Phys Fluids A. Vol. 1(3):507512.

Bear, J. 1972. Dynamics of fluids in porous media. American Elsevier, New York.

Berkowitz, B., Cortis, A., Dentz, A., and Scher, H. 2006. Modeling Non-Fickian Transport in Geological Formations. Rev. Geophys. Vol. 44, doi:10.1029/2005RG000178.

Bhatnagar, P., E.P. Gross., and M.K. Krook. 1954. A model for collision processes in gases: I. small amplitude processes in charged and neutral one-component system. Phys. Rev. E. Vol. 94 (3):511-525. 
Birk, S., T. Geyer., R. Liedl., and M. Sauter. 2005. Process-based interpretation of tracer tests in carbonate aquifers. Ground Water. Vol. 43 (3): 381-388.

Brenner, H. 1962. The diffusion model of longitudinal mixing in beds of finite length. Numerical values. Chem. Eng. Sci. Vol. 17:229-243.

Brigham, W.E. 1974. Mixing equations in short laboratory cores. Soc. Pet Eng. J. Vol. 14:91-99.

Brown, M.C. and D.C. Ford. 1971. Quantitative tracer methods for investigations of karst hydrology systems, with special reference to the Maligne Basin area, Canada. Transactions of the Cave Research Group of Great Britain. Vol. 13 (1): 37-51.

Camas Serván, B. 2007. Saltwater Intrusion Simulation in Heterogeneous Aquifer Using Lattice Boltzmann Method, M.S. Thesis, Louisiana State University, 77 pp.

Chen, H. 1998. Volumetric formulation of the lattice Boltzmann method for fluid dynamics: Basic concept. Phys Rev E. Vol. 56: 3955-3963.

Chen, S. and G. Doolen. 1998. Lattice Boltzmann method for fluid flows. Annu. Rev. Fluid. Mech. Vol. 30: 329-364.

Chen, S., G. Doolen, and K. Eggert. 1994. Lattice-Boltzmann Fluid Dynamics - A Versatile Tool for Multiphase and Other Complicated Flows. Los Alamos Science Vol. 22: 98-111.

Clemens, T., D. Hueckinghaus, M. Sauter, R. Liedl, and G. Teutsch. 1997. Modelling the genesis of karst aquifer systems using a coupled reactive network model. Hardrock Hydrosystems. Vol. 241:3-10.

Cleary, R.W. and D.D. Adrian. 1973. Analytical solution of the convective dispersive equation for cation adsorption in soils. Soil Sci. Am. Proc. Vol. 37:197-199

Coats, K.H. and B.D. Smith. 1964. Dead end pore volume and dispersion in porous media. Soc Pet Eng Jour. Vol. 4:73-84.

Commission on Geosciences, Environment and Resources (CGER). 1996. Rock Fractures and Fluid Flow: Contemporary Understanding and Applications. National Academy Press.

Cortis, A. and Berkowitz, B. 2004 Anomalous Transport in "Classical" Soil and Sand Columns. Soil. Sci. Soc. Am. J. Vol. 68: 1539-1548.

Dardis, O. and J. McCloskey., 1998 a. Lattice Boltzmann scheme with real numbered solid density for the simulation of flow in porous media. Phys Rev E. Vol. 57 (4): 4834-4837. 
Dardis, O. and J. McCloskey., 1998 b. Permeability porosity relationships from numerical simulations of fluid flow. Geophys. Res. Let. Vol. 25 (9): 1471-1474.

Diersch, H. J. G. 2002. Discrete feature modeling of flow, mass and heat transport processes by using FEFLOW. White Papers 1. Chapter 9, WASY Ltd. 147-190.

DiFrenna, V. J. 2005. Effect of scaling on hydraulic conductivity in a karst aquifer. M.S. Thesis. Florida International University.

Drew, D. 1995. Glossary of karstic terminology. In: COST Action 65 Report Hydrogeological aspects of groundwater protection in karstic areas. European Commission. 446p.

Field, M. S. 1993. Karst hydrology and chemical contamination. J Environ. Syst. Vol. 22 (1):1-26.

Field, M. S. 1999. The QTRACER Program for Tracer-Breakthrough Curve Analysis for Karst and Fractured-Rock Aquifers. Environmental Protection Agency.

Field, M.S. and P.F. Pinsky. 2000. A two-region nonequilibrium model for solute transport in solution conduits in karstic aquifers. Jour Cont Hydro. Vol. 44 (3-4): 329-351.

Fillipova, O. and D. Hänel. 1998. Grid refinement for Lattice-BGK models. Jour Comp Phy. Vol. 147: 219-228.

Flekkøy, E. G. 1993. Lattice Bhatnagar-Gross-Krook models for miscible fluids. Phy Rev E. Vol. 47 (6): 4247-4257.

Fried J. J. and M. A. Combarnous. 1971. Dispersion in porous media. Adv. Hydrosci. Vol. 7: 169

Freed, D. M. 1998 Lattice-Boltzmann methods for macroscopic porous media modeling. Int. J. Mod. Phys. C. Vol. 9: 1491-1503.

Freeze, R. A. and J. A. Cherry. 1979. Groundwater. Prentice Hall. pp.394.

Gao, Y., and M. M. Sharma. 1994. A LGA model for fluid flow in heterogeneous porous media. Transport Porous Med. Vol. 17:1-17.

Geyer, T., S. Birk, T. Licha, R. Liedl and M. Sauter. 2007. Multitracer test approach to characterize reactive transport in karst aquifers. Ground water. Vol. 45 (1): 36-45.

Ginzburg, I. 2005 Equilibrium-type and link-type lattice Boltzmann models for generic advection and anisotropic-dispersion equation. Adv. Water. Resour. Vol. 28 (11): 1171-1195 
Goldscheider, N. 2008. A new quantitative interpretation of the long-tail and plateau-like breakthrough curves from tracer tests in the artesian karst aquifer of Stuttgart, Germany. Hydrogeology Journal. doi: 10.1007/s10040-008-0307-0

Goldstein, F. R. S. 1953. On the mathematics of exchange processes in fixed columns. I. Mathematical solutions and asymptotic expansions. Proc. Roy. Soc. London. Vol. 219:151-185.

Göppert, N., and N. Goldscheider. 2008. Solute and Colloid Transport in Karst Conduits under Low-and High-Flow Conditions. Ground Water Vol. 46 (1): 61-68.

Grubert, D. 1997. Using FHP-BGK-Model to get effective dispersion constants for spatially periodic model geometries. Int J Mod Phys C. Vol. 8(4):817-25.

Green R., T. S. L. Painter., A. Sun., S. R. H. Worthington. 2006. Groundwater contamination in karst terranes. Water, Air, and Soil Pollution. Vol. 6:157-170.

Hassanizadeh, S. M. 1996. On the transient non-Fickian dispersion theory. Transport Porous Media. Vol. 23: 107-124.

He, Y. L. S. Luo and M. Dembo. 1996. Some progress in Lattice Boltzmann method. Part I. Nonuniform mesh grids. J. Comp Phys. Vol. 129: 357-363.

Henry, H. R. 1964. Effects of dispersion on salt encroachment in coastal aquifers. US Geological Survey Water Supply Paper. Vol. 1613-C: C71-C84.

Howard, A. D. and C. G. Groves. 1995. Early development of karst systems 2: Turbulent flow. Wat Resour Res. Vol. 31 (1): 19-26.

Huntoon, P.W., 1995. Is it appropriate to apply porous media groundwater circulation models to karstic aquifers? In: El-Kadi, A.I., (Ed.), Groundwater Models for Resources Analysis and Management, 1994 Pacific Northwest/Oceania Conference, Honolulu, HI. Boca Raton, Florida. Lewis Publishers. 339-358.

Inamuro, T., M. Yoshino, H. Inoue, R. Mizuno, and F. Ogino. 2002. A lattice Boltzmann method for a Binary Miscible Fluid Mixture and its application to a Heat-Transfer Problem. J. Comp Phys. Vol. 179: 201-215.

James, R. V. and J. Rubin. 1972. Accounting for apparatus-induced dispersion in analyses of miscible displacement experiments. Water Resour. Res. Vol. 8 (3): 717-721.

Kang, Q., D. Zhang and S. Chen. 2002. Unified lattice Boltzmann method for flow in multiscale porous media. Phy Rev E. Vol. 66: 056307.

Kincaid, T. R. 1999. Morphologic and Fractal Characterization of saturated karstic caves. Ph. D. Dissertation. University of Wyoming. 
Kreft, A. and A. Zuber. 1978. On the physical meaning of the dispersion equation and its solutions for different initial and boundary conditions. Chem Eng Sci. Vol. $33: 1471-1480$

Kutilek, M and D.R. Nielsen., 1994. Soil Hydrology. P 274-285. Catena, CremligenDrestedt, Germany.

Lapidus, L., and N. R. Amundson. 1952. The mathematics of adsorption in beds. IV. The effect of longitudinal diffusion in ion exchange chromatographic columns. J. Phys Chem. Vol. 56:984-988.

Lindstrom, F. T., R. Hague, V. H. Freed, and L. Boersma. 1967. Theory on the movement of some herbicides in soils: linear diffusion and convection of chemicals in soils. Environ. Sci. Technol. Vol. 1:561-565.

Lohman. S. W. 1972. Ground water hydraulics. Geological survey professional paper708. pp:70.

Martin, J. B., and E. J. Screaton. 2001. Exchange of matrix and conduit water with examples from the Floridan aquifer. Proceedings of the U.S. Geological Survey Karst Interest Group. Water-Resources Investigations Report 01-4011: 38-44.

Martys, N. S. 2001. Improved approximation of the Brinkman equation using a lattice Boltzmann method. Phy Fluids. Vol. 13(6): 1807-1809.

Massei, N. H. Q. Wang, M. S. Field, J. P. Dupont, M. Bakalowicz, and J. Rodet. 2006. Interpreting tracer breakthrough tailing in a conduit-dominated karst aquifer. Hydrogeology Journal. Vol. 14: 849-858.

Maloszweski, P. W. Stchler, A. Zuber, D. Rank. 2002. Identifying the flow systems in a karstic-fissured-porous aquifer, the Schneealpe, Austria, by modeling of environmental $18 \mathrm{O}$ and $3 \mathrm{H}$ isotopes. Journal of Hydrology. Vol. 256: 48-59.

Mull, D.S., T.D. Liebermann, J. L. Smoot, and L. H. Woosley Jr. 1988. Application of dye-tracing techniques for determining solute-transport characteristics of ground water in karst terranes, Rep. EPA 904r6-88-001, US Environmental Protection Agency, Atlanta, GA, 103 p.

Narasimhan, T. N. 1999. Fourier's heat conduction equation: History, influence, and connections. Rev. Geophys. Vol. 37(1): 151-172. doi:10.1029/1998RG900006

Novakowski, K. S. 1992. An evaluation of boundary conditions for one-dimensional solute transport 1. Mathematical development. Wat. Resour. Res. Vol. 28 (9): 2399-2410. 
Osiensky, J. L., R. E. Williams., B. Williams and G. Johnson. 2000. Evaluation of drawdown curves derived from multiple well aquifer tests in heterogeneous environments. Mine Water and the Environment. Vol. 19(1): 30-55.

Neuman, S. P. 1987. Stochastic continuum representation of fractured rock permeability as an alternative to the REV and fracture network concepts. Groundwater flow and quality modeling. Reidel Publ. Dordecht. 331-362.

Palmer A.N., 2000, Hydrogeologic control of cave patterns: In Klimchouk, A., Ford, D., Palmer, A., and Dreybrodt, W., eds. Speleogenesis: evolution of karst aquifers, National Speleological Society. 77-90.

Parker, J. C. 1984. Analysis of solute transport in column tracer studies, Soil Sci. Soc. Amer. J. Vol. 48: 719-724.

Parker, J. C., and M. Th. van Genuchten. 1984. Determining transport parameters from laboratory and field tracer experiments, Bull. 84-3, Va Agric. Exp. St., Blaacksburg, Va,

Perea-Reeves, S.J., and H.W. Stockman. 1997. A lattice-gas study of dispersion in alveolated channels. Chem Eng Sci. Vol. 52 (9): 3277-3286

Qian Y., D. d'Humieres, and P. Lallemand. 1992. Lattice BGK models for Navier-Stokes equation. Europhys. Lett. Vol. 17:479-484.

Quinn, J. J. and D. Tomasko. 2000. A numerical approach to simulating mixed flow in karst aquifers, in Sasowsky, I.D. \& Wicks, C.M., ed., Groundwater flow and contaminant transport in carbonate aquifers: Rotterdam, Balkema. 147-156.

Quinn, J. J., D. Tomasko., and J. A. Kuiper. 2006. Modeling complex flow in a karst aquifer. Sedimentary Geology. Vol. 184: 343-351.

Resse, R. S. and M. Wacker. 2007. Pump test of well PB-1545 in Palm Beach County. (Personal communication).

Renken, R. A., K. J. Cunningham., A. M. Shapiro, R. W. Harvey. 2008. Pathogen and Chemical Transport in the Karst Limestone of the Biscayne Aquifer: 1. Revised Conceptualization of Groundwater Flow. Wat Resour Res. doi:10.1029/2007WR006058 (in press).

Sauter, M. 1993. Quantification and forecasting of regional ground water flow and transport in karst aquifer. Ph.D dissertation at the Geowissenschaftliche fakultat, Universitat Tubingen, Germany.

Scanlon, B.R., R.E. Mace, M.E. Barrett, and B. Smith. 2003. Can we simulate regional groundwater flow in a karst system using equivalent media models? Case study Barton Springs Edwards Aquifer, USA. J Hydrol Vol. 276(1-4): 137-158. 
Schwartz F. W and H. Zhang. 2003. Fundamentals of Ground Water. Wiley. USA.

Scott, T.M., G.H. Means, R.C. Means, and R.P. Meegan 2002. First Magnitude Springs of Florida. Open File Report No. 85. Florida Geological Survey, Tallahassee, Florida

Sharp, J. C., and Y. N. T. Maini. 1972. Fundamental considerations on the hydraulic characteristics of joints in Rock. Paper T1-F, International Symposium on Percolation through Fissured Rock, Stuttgart.

Shim, W. G, D. Y. Choi, S. P. Choi, J. W. Lee, H. Moon. 2005. Column Dynamics of Benzene Vapor Adsorption on MCM-48. Adsorption. Vol. 11: 503-507.

Shoemaker, W. B., K. J. Cunningham, E. Kuniansky, and J. Dixon. 2008. Effects of turbulence on hydraulic heads and parameter sensitivities in preferential groundwater flow layers, Wat Resour Res. doi:10.1029/2007WR006601.

Shu, C. Y. T. Chew and X. D. Niu. 2001. Least-squares-based lattice Boltzmann method: A meshless approach for simulation of flows with complex geometry. Phy. Rev. E. Vol. 64 (4): 045701-1045701-4.

Simunek, J., M. Th. van Genuchten., M. Sejna., N. Toride., and F. J. Leij. 1999. The STANMOD computer software for evaluating solute transport in porous media using analytical solutions of convection-dispersion equation. Versions 1.0 and 2.0, IGWMC - TPS - 71, International Ground Water Modeling Center, Colorado School of Mines, Golden, Colorado, 32 pp.

Smart, C. C., and D. C. Ford. 1986. Structure and foundation of a conduit aquifer. Can J Earth Sci. Vol. 23(7): 919-929.

Smart, C.C. 1988. Artificial tracer techniques for the determination of the structure of conduit aquifers. Ground Water Vol. 26 (4): 445-453.

Spaid, M. A. A., and F. R. Phelan, Jr. 1997. Lattice Boltzmann methods for modeling microscale flow in fibrous porous media. Phys. Fluids. Vol. 9(9): 2468-2474.

Stockman HW, Glass RJ, Cooper C, Rajaram H 1998 Accuracy and computational efficiency in 3D dispersion via lattice Boltzmann: models for dispersion in rough fractures and double-diffusive fingering. Int. J. Modern. Phys. C. Vol. 9:15451557

Succi, S. 2001. The Lattice Boltzmann Equation: For Fluid Dynamics and Beyond. Oxford University Press, New York. ISBN: 0198503989

Sun, A. and Painter, S. L.: 2004, 'A MODFLOW-Based Approach for Modeling Flow in Karst Aquifers', Proceedings of 2004 GSA Annual Meeting and Exposition, Denver, CO, USA. 
Sun A. Y., S. L. Painter., and R. T. Green. 2005. Modeling Barton Springs segment of the Edwards aquifer using MODFLOW-DCM. Proceedings of Sinkholes and the Engineering and Environmental Impacts of Karst. 163-168.

Sukop M.C., and D.T. Thorne., 2006. Lattice Boltzmann Modeling : An Introduction for Geoscientists and Engineers. Springer. Berlin. pp. 172

Sukop, M.C., S. Anwar, J.S. Lee, K.J. Cunningham, and C.D. Langevin. 2008. Modeling Ground-water Flow and Solute Transport in Karst with Lattice Boltzmann Methods, Proceedings of the U.S. Geological Survey Karst Interest Group Workshop, Bowling Green, Kentucky, Western Kentucky University Campus. May 27-29, 2008. Scientific Investigations Report 2008-5023: 77-86.

Sullivan, S.P., F.M. Sani, M.L. Johns, and L. F. Gladden. 2005. Simulation of packed bed reactors using lattice Boltzmann methods. Chem Eng. Sc. Vol. 60: 3405-3418.

Taylor, G.I. 1954. The dispersion of matter in turbulent flow through a tube. Proc. Royal. Soc. London A. Vol. 225:446-468.

Theis, C. V. 1935. The relation between the lowering of the piezometric surface and the rate and duration of discharge of a well using ground-water storage. EOS, Vol. 16 (2): 519-524.

Thorne, D.T. and Sukop, M.C., 2004, Lattice Boltzmann model for the Elder problem, In Proceedings of the XVth International Conference on Computational Methods in Water Resources (CMWR XV), June 13-17, 2004, Chapel Hill, NC, USA. C.T. Miller, M.W. Farthing, W.G. Gray, and G. F. Pinder Eds. Elsevier, Amsterdam.

Toride, N. and F. J. Leji. 1996. Convective-Dispersive stream tube model for field scale solute transport: I. Model Analysis. Soil Sci. Soc. Am. J. Vol. 60: 342-352.

Toride, N., F. J. Leji., M. Th. van Genuchten. 1999. The CXTFIT code for estimating transport parameters from laboratory and field tracer experiments, Research Report no. 137/1999, U.S. Salinity Laboratory, Riverside, California.

Tritton, D.J. 1988. Physical fluid dynamics. 2nd ed. Oxford publ. NY.

van Genuchten, M. Th., and J.C. Parker. 1984. Boundary conditions for displacement experiments through short laboratory soil columns. Soil Sci. Soc. Am. J. Vol. 48:703-708.

van Genuchten, M. Th., and P.J. Wierenga . 1986. Soil dispersion coefficients and retardation factors in methods of soil analysis, part 1. Physical and mineralogical methods-Agronomy monograph no. 9. 1025:1054. Eds Madison Wisconsin, USA. 
Walsh, S. D. C., H. Burwinkle and M.O. Saar. 2008. A new partial-bounceback Lattice Boltzmann method for fluid flow through heterogeneous media. Computers and Geosciences (in press).

Wang, H. F. and M. P. Anderson. 1982. Introduction to groundwater modeling: finite difference and finite element methods. Academic press. pp-89.

Watson, V., R. Painter., and T. Byl. 2003. Numerical Simulation of Flow and Contaminant Transport in a Complex Karst Conduit. Society of Environmental Toxicology and Chemistry. 24th Annual meeting. TX.

White, B. 2002. Karst hydrology: recent developments and open questions. Eng. Geol. Vol. 65: 85-105.

White, W.B. and E.L.White. 2005. Ground water flux distribution between matrix, fractures, and conduits: constraints on modeling. Speleogenesis and Evolution of Karst Aquifers. Vol. 3 (2): 2-8.

Wolf-Gladrow, D. A. 2000. Lattice gas cellular automata and lattice Boltzmann models: an introduction. Lecture notes in Mathematics. Springer. Berlin. pp. 308.

Yoshino, M. and T. Inamuro. 2003. Lattice Boltzmann simulations for flow and heat mass transfer problems in three-dimensional porous structure. Int. J. Num. Meth. Fluids. Vol. 43: 183-198.

Zhang, X., A. G. Bengough., J. W. Crawford., I. M. Young. 2002 a. A lattice BGK model for advection and anisotropic dispersion equation. Adv Wat Res. Vol. 25:1-8.

Zhang, X., J.W. Crawford., A.G. Bengough., and I.M. Young. 2002 b. On boundary conditions in the lattice Boltzmann model for advection and anisotropic dispersion equation, Adv in Water Res Vol. 25 (6): 601-609

Zheng, C. 2006. MT3DMS Supplemental User's Guide, Technical Report to the U.S. Army Engineer Research and Development Center, v5.2 Department of Geological Sciences, University of Alabama, pp. 24.

Zhou, J. G. 2007. A rectangular Lattice Boltzmann method for groundwater flows. Modern Physics Letters B Vol. 21 (9): 531-542.

Zou, Q. and X. He. 1997. On pressure and velocity boundary conditions for the lattice Boltzmann BGK model. Physics of Fluids Vol. 9 (6): 1591-1598. 
VITA

\section{EDUCATION}

\section{SHADAB ANWAR}

Masters of Engineering, Civil and Environmental Engineering July 2003

Thesis title: Spatial moment analysis of reactive contaminant transport in open channel with porous bed.

Indian Institute of Technology Kanpur India

Bachelor of Engineering, Civil and Environmental Engineering May 2001

Muzaffarpur Institute of Technology Muzaffarpur India

\section{PUBLICATIONS}

Anwar, S. and M.C. Sukop. 2008. Lattice Boltzmann models for flow and transport in phreatic karst. (accepted in Ground Water).

Anwar, S. and M.C. Sukop. 2008. Regional scale transient groundwater flow modeling using lattice Boltzmann methods (accepted in Progress in Computational Fluid Dynamics).

Anwar, S., A. Cortis, and M.C. Sukop. 2008. Lattice Boltzmann Simulation of Solute Transport in Heterogeneous Porous Media with Conduits to Estimate Macroscopic Continuous Time Random Walk Model Parameters. Progress in Computational Fluid Dynamics. Vol. 8 (1-4) pp. 213 - 221. DOI: 10.1504/PCFD.2008.018092

Sukop M.C., S. Anwar, J.S. Lee, K.J. Cunningham, and C.D. Langevin. 2008. Modeling Ground-water Flow and Solute Transport in Karst with Lattice Boltzmann Methods. Proceedings of the U.S. Geological Survey Karst Interest Group Workshop, May 2729, 2008, Bowling Green, Kentucky, Western Kentucky University Campus.

Scientific Investigations Report 2008-5023: 77-86.

Bardsley, K.J., S. Anwar, and M.C. Sukop. 2006. Simultaneous heat and solute transport modeling of ground water with lattice Boltzmann methods. CMWR XVI -

Computational Methods in Water Resources, XVI International Conference,Copenhagen, Denmark, June 19-22

\section{CONFERENCES}

Anwar, S. and M. C. Sukop. 2008 Lattice Boltzmann Methods for Fluid and Solute Transport Modeling in Karst Aquifers. EOS Trans. AGU, 89 (23), Jt Assem. Suppl., Abstract H33D-08 (Oral presentation)

Anwar, S. and M. C. Sukop. 2008 Lattice Boltzmann Methods for Fluid and Solute Transport Modeling in Karst Aquifers. International Conference for Mesoscopic Methods in Engineering and Scinece. Amsterdam, June 16-20 (Oral Presentation)

Anwar, S. and M. C. Sukop. 2007 Regional scale flow modeling using Lattice Boltzmann models. EOS Trans. AGU, 88 (52), Fall Meet. Suppl., Abstract H33D-1624 
Anwar, S. and M.C. Sukop 2007 Verification of lattice Boltzmann models for solute transport modeling in karst aquifers. International Conference for Mesoscopic Methods in Engineering and Scinece. Germany, July 16-20

Anwar, S., A. Cortis, and M.C. Sukop 2006 Lattice Boltzmann Simulation of Solute Transport in Heterogeneous Porous Media with Conduits to Estimate Macroscopic Continuous Time Random Walk Model Parameters. EOS Trans. AGU, 87 (52), Fall Meet. Suppl., Abstract H21C-1384

Sukop, M.C., S. Anwar, and Katie Bardsley 2005 Transport in large scale porous media with conduits via lattice Boltzmann models. EOS Trans. AGU, 86 (52), Fall Meet. Suppl., Abstract H42A-04

Anwar, S. and R. Shrivastava 2005 Spatial Moment Analysis of Reactive Contaminant Transport in Open Channel with Porous Bed. EOS Trans. AGU, 86 (52), Fall Meet. Suppl., Abstract. H31D-1340.

Sukop, M.C., D. T. Thorne, and S. Anwar 2004 Lattice Boltzmann Methods and their Boundary Conditions for Solute Transport. EOS Trans. AGU, 85 (47), Fall Meet. Suppl., Abstract H32A-07.

SKILLS

Programming Languages: C/C++, FORTRAN77

Libraries and Tools: MATLAB, Mathematica, MODFLOW, MT3D, GIS, GCC, Vim, LATEX, MPI (MPICH2), Python

\section{HONORS}

- $\quad$ Presidential Dissertation Year Fellowship (DYF) award during the last year of Ph.D.

- $\quad$ Received NSF travel grants to attend and present at the International Conference of Mesoscopic Methods in Engineering and Sciences (ICMMES) in 2006, 2007 and 2008. 